\title{
Kinetic Resolution of Hydroperoxides with Enantiopure Phosphines: Preparation of Enantioenriched Tertiary Hydroperoxides
}

\author{
Tom G. Driver, Jason R. Harris and K. A. Woerpel \\ Contribution from the Department of Chemistry, University of California, \\ Irvine, CA 92697-2025
}

Supporting Information

\section{Contents:}

I. Preparation of Alcohols 12, 28, 30, 32, 34, 36, 38, 39, $41 \quad$ S-2

II. Preparation of Hydroperoxides 11, 13-24 S-5

III. Preparation of Xylyl-PHANEPHOS $10 \quad$ S-11

IV. Kinetic Resolutions $\quad$ S-14

$\begin{array}{lll}\text { V. Isolation of Enantiopure Hydroperoxide } & \text { S-19 }\end{array}$

VI. Resolution with Mono(phosphine oxide) $25 \quad$ S-21

VII. Determination of Absolute Configuration and Rotation $\quad$ S-23

VIII. SFC and HPLC traces and NMR Spectra S-26

General. NMR spectra were recorded in $\mathrm{CDCl}_{3}$ at ambient temperature. ${ }^{1} \mathrm{H}$ NMR spectra were measured at $500 \mathrm{MHz}$ on a Bruker CRYO500 or GN500 and were referenced to residual $\mathrm{CHCl}_{3}(7.27 \mathrm{ppm}) .{ }^{13} \mathrm{C}$ NMR spectra were measured at $125 \mathrm{MHz}$ on a Bruker CRYO500 or GN500 and were referenced to the centerline of the $\mathrm{CDCl}_{3}$ triplet $(77.23 \mathrm{ppm}) .{ }^{31} \mathrm{P} \mathrm{NMR}$ spectra were measured at $162 \mathrm{MHz}$ and referenced externally to $85 \% \mathrm{H}_{3} \mathrm{PO}_{4}(0.0 \mathrm{ppm})$. The NMR spectral data are reported as follows: chemical shift in ppm on the $\delta$ scale, multiplicity $(\mathrm{br}=$ broad, $\mathrm{s}=$ singlet, $\mathrm{d}=$ doublet, $\mathrm{t}=$ triplet, $\mathrm{q}=$ quartet, $\mathrm{qu}=$ quintuplet, $\mathrm{sp}=$ septet, $\mathrm{m}=$ multiplet $)$, coupling constants $(\mathrm{Hz})$ and integration. Infrared (IR) spectra were obtained using a MIDAC Prospect FT-IR spectrometer. High-resolution mass spectra were acquired on a VG Analytical $7070 \mathrm{E}$ or Fisions Autospec spectrometer, and were obtained by peak matching. Atlantic Microlab, Atlanta, GA, performed microanalyses. Melting points are reported uncorrected. Liquid chromatography was performed using forced flow (flash chromatography) of the indicated solvent system in EM Reagents silica gel $\left(\mathrm{SiO}_{2}\right) 60$ (230-400 mesh). Enantiomeric excess was determined using a Berger SFC Analytix or a Hewlett Packard series 1100 HPLC, and the enantiomerically enriched material was compared to racemic material (see note below). Optical rotations were measured on a JASCO P-1010 polarimeter. Unless otherwise noted, all reactions were performed open to air and all reagents were obtained from commercial sources and used without further purification. $\mathrm{Et}_{2} \mathrm{O}$ and $\mathrm{THF}$ were dried by filtration through alumina according to the procedure of Grubbs. ${ }^{1}$ 
Caution: Although we have not had any incidents exploring this chemistry, caution should be exercised when handling organic peroxides: scales should be minimized, safety shields should always be employed and precautions should be taken to minimize exposure to light, heat and metal salts. ${ }^{2}$ In addition, in the syntheses of hydroperoxides, the order of addition of the reagents is crucial to avoid uncontrollable chain reactions; alcohols should be added to a mixture of dilute acid in $\mathrm{H}_{2} \mathrm{O}-\mathrm{H}_{2} \mathrm{O}_{2}$. Concentrated acid should never be added directly to an alcohol-hydrogen peroxide- $\mathrm{H}_{2} \mathrm{O}$ mixture, as explosions have been known to occur. ${ }^{3}$

\section{Preparation of Alcohols 12, 28, 30, 32, 34, 36, 38, 39, 41}

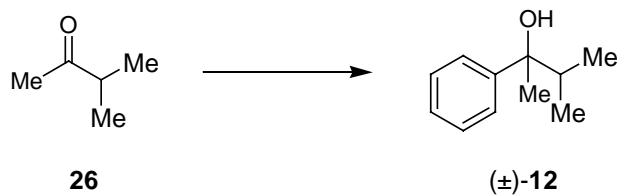

( \pm )-3-Methyl-2-phenylbutan-2-ol (12). ${ }^{4}$ To a cooled $\left(-78{ }^{\circ} \mathrm{C}\right)$ solution of bromobenzene $(7.9 \mathrm{~mL}, 75 \mathrm{mmol})$ in THF $(250 \mathrm{~mL})$ was added $n$-BuLi $(32 \mathrm{~mL}, 79 \mathrm{mmol}, 2.5 \mathrm{M}$ solution in hexane). The mixture was stirred for one hour and 3-methylbutan-2-one (26) $(9.7 \mathrm{~mL}, 90 \mathrm{mmol})$ was added over one-half hour. The reaction mixture was stirred at ambient temperature for one hour and partitioned between $\mathrm{Et}_{2} \mathrm{O}(100 \mathrm{~mL})$ and water $(100$ $\mathrm{mL})$. The aqueous layer was extracted with $\mathrm{Et}_{2} \mathrm{O}(2 \times 50 \mathrm{~mL})$. The combined organic layers were washed with brine $(50 \mathrm{~mL})$ and dried over $\mathrm{MgSO}_{4}$. The resulting mixture was filtered and the filtrate was concentrated in vacuo. The resulting oil was purified by flash chromatography (hexanes/EtOAc 9:1) yielding alcohol $12(8.0 \mathrm{~g}, 65 \%)$ as a clear oil. Spectral data matched those reported in the literature: ${ }^{5}{ }^{1} \mathrm{H}$ NMR $\left(500 \mathrm{MHz}, \mathrm{CDCl}_{3}\right) \delta$ 7.49-7.51 (m, 2H), 7.32-7.42 (m, 2H), 7.23-7.29 (m, 1H), 2.08-2.11 (m, 1H), $1.78(\mathrm{~s}$, $1 \mathrm{H}), 1.59(\mathrm{~s}, 3 \mathrm{H}), 0.97(\mathrm{~d}, J=6.7,3 \mathrm{H}), 0.88(\mathrm{~d}, J=6.8,3 \mathrm{H}) ;{ }^{13} \mathrm{C} \mathrm{NMR}(125 \mathrm{MHz}$, $\left.\mathrm{CDCl}_{3}\right) \delta 148.3,128.3,126.9,125.7,77.0,39.1,27.1,17.9,17.8$. Anal. Calcd for $\mathrm{C}_{11} \mathrm{H}_{16} \mathrm{O}: \mathrm{C}, 80.44 ; \mathrm{H}, 9.82$. Found: C, 80.27; H, 10.03 .

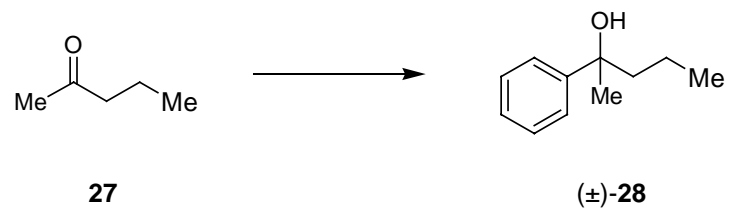

( \pm )-2-Phenylpentan-2-ol (28). The procedure for the synthesis of alcohol $( \pm)-12$ was followed using 2-pentanone (27) $(5.1 \mathrm{~mL}, 48 \mathrm{mmol})$, bromobenzene $(5.0 \mathrm{~mL}, 48 \mathrm{mmol})$, $n$-BuLi (34 mL, $48 \mathrm{mmol}, 1.4 \mathrm{M}$ solution in hexane) to afford alcohol 28 as a clear oil $(5.2 \mathrm{~g}, 67 \%)$ after purification by flash chromatography (hexanes/EtOAc 19:1). Spectral data matched those reported in the literature: ${ }^{6} \mathrm{H}_{\mathrm{NMR}}\left(500 \mathrm{MHz}, \mathrm{CDCl}_{3}\right) \delta 7.49-7.51$ $(\mathrm{m}, 2 \mathrm{H}), 7.39-7.42(\mathrm{~m}, 2 \mathrm{H}), 7.28-7.32(\mathrm{~m}, 1 \mathrm{H}), 1.79-1.87(\mathrm{~m}, 2 \mathrm{H}), 1.79(\mathrm{~s}, 1 \mathrm{H}), 1.62(\mathrm{~s}$, $3 \mathrm{H}), 1.22-1.37(\mathrm{~m}, 2 \mathrm{H}), 0.93(\mathrm{t}, J=7.5,3 \mathrm{H}) ;{ }^{13} \mathrm{C} \mathrm{NMR}\left(125 \mathrm{MHz}, \mathrm{CDCl}_{3}\right) \delta 148.1$, 128.2, 126.5, 124.8, 74.8, 46.6, 30.2, 17.3, 14.5; IR (thin film) 3417, 2935, 1951, 1885 , 1447, $1029 \mathrm{~cm}^{-1}$. Anal. Calcd for $\mathrm{C}_{11} \mathrm{H}_{16} \mathrm{O}: \mathrm{C}, 80.44 ; \mathrm{H}, 9.82$. Found: C, 80.41; H, 10.10 . 


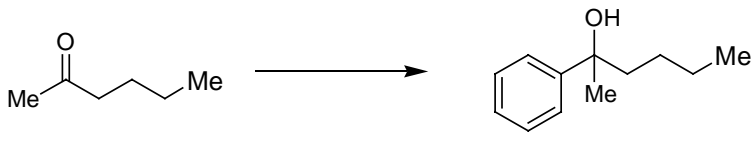

$( \pm)-30$

( \pm )-2-Phenylhexan-2-ol (30). The procedure for the synthesis of alcohol ( \pm )-12 was followed using 2-pentanone (29) (4.8 g, $48 \mathrm{mmol})$, bromobenzene (5.0 mL, $48 \mathrm{mmol}), n$ BuLi (19 mL, $48 \mathrm{mmol}, 2.5 \mathrm{M}$ solution in hexane) to afford alcohol $\mathbf{3 0}$ as a clear oil (5.3 g, 63\%) after purification by flash chromatography (hexanes/EtOAc 19:1). Spectral data matched those reported in the literature: ${ }^{7}{ }^{1} \mathrm{H}$ NMR $\left(500 \mathrm{MHz}, \mathrm{CDCl}_{3}\right) \delta 7.43-7.45(\mathrm{~m}$, $2 \mathrm{H}), 7.33-7.37(\mathrm{~m}, 2 \mathrm{H}), 7.23-7.26(\mathrm{~m}, 1 \mathrm{H}), 1.78-1.84(\mathrm{~m}, 2 \mathrm{H}), 1.72(\mathrm{~s}, 1 \mathrm{H}), 1.57(\mathrm{~s}, 3 \mathrm{H})$, $1.23-1.30(\mathrm{~m}, 3 \mathrm{H}), 1.11-1.14(\mathrm{~m}, 1 \mathrm{H}), 0.86(\mathrm{t}, J=7.0,3 \mathrm{H}) ;{ }^{13} \mathrm{C} \mathrm{NMR}\left(125 \mathrm{MHz}, \mathrm{CDCl}_{3}\right)$ $\delta 148.1,128.2,126.5,124.8,74.8,44.0,30.2,26.2,23.2,14.1$; IR (thin film) 3417, 3027, 2958, 1603, 1495, $1028 \mathrm{~cm}^{-1}$; HRMS (EI) $\mathrm{m} / \mathrm{z}$ calcd for $\mathrm{C}_{12} \mathrm{H}_{18} \mathrm{O}[\mathrm{M}+\mathrm{Na}]^{+} 201.1255$, found 201.1253.

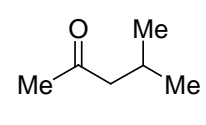

31

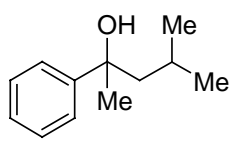

$( \pm)-32$

( \pm )-4-Methyl-2-phenylpentan-2-ol (32). The procedure for the synthesis of alcohol $( \pm)$ 12 was followed using 4-methyl-2-pentanone (31) $(5.9 \mathrm{~mL}, 48 \mathrm{mmol})$, bromobenzene (5.0 mL, $48 \mathrm{mmol}), n$-BuLi $(20 \mathrm{~mL}, 48 \mathrm{mmol}, 1.4 \mathrm{M}$ solution in hexane) to afford alcohol 32 as a clear oil (4.3 g, 50\%) after purification by flash chromatography (hexanes/EtOAc 19:1). Spectral data matched those reported in the literature: ${ }^{8}{ }^{1} \mathrm{H}$ NMR $\left(500 \mathrm{MHz}, \mathrm{CDCl}_{3}\right) \delta$ 7.45-7.46 (m, 2H), 7.33-7.37 (m, 2H), 7.23-7.27 (m, 1H), 1.69-1.83 $(\mathrm{m}, 4 \mathrm{H}), 1.57(\mathrm{~s}, 3 \mathrm{H}), 0.89(\mathrm{~d}, J=6.6,3 \mathrm{H}), 0.77(\mathrm{~d}, J=6.5,3 \mathrm{H}) ;{ }^{13} \mathrm{C}$ NMR $(125 \mathrm{MHz}$, $\left.\mathrm{CDCl}_{3}\right) \delta 148.3,128.1,126.5,124.9,75.3,52.8,31.4,24.5,24.4$; IR (thin film) 3449, 2956, 2717, 1602, 1446, $1028 \mathrm{~cm}^{-1}$. Anal. Calcd for $\mathrm{C}_{12} \mathrm{H}_{18} \mathrm{O}: \mathrm{C}, 80.85 ; \mathrm{H}, 10.18$. Found: C, 80.59; H, 10.28.

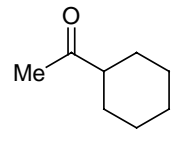

33

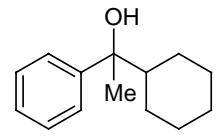

$( \pm)-34$

( \pm )-1-Cyclohexyl-1-phenylethanol (34). The procedure for the synthesis of alcohol ( \pm )12 was followed using 1-cyclohexylethanone (33) $(6.0 \mathrm{~mL}, 48 \mathrm{mmol})$, bromobenzene (5.0 mL, $48 \mathrm{mmol}$ ), $n$-BuLi $(37 \mathrm{~mL}, 52 \mathrm{mmol}, 2.4 \mathrm{M}$ solution in hexane) to afford alcohol 34 as a clear oil (4.1 g, 46\%) after purification by flash chromatography (hexanes/EtOAc 19:1). Spectral data matched those reported in the literature: ${ }^{9}{ }^{1} \mathrm{H}$ NMR $\left(500 \mathrm{MHz}, \mathrm{CDCl}_{3}\right) \delta$ 7.40-7.43 (m, 2H), 7.32-7.36 (m, 2H), 7.23-7.26 (m, 1H), $2.14(\mathrm{~s}$, $1 \mathrm{H}), 1.54-1.77(\mathrm{~m}, 5 \mathrm{H}), 1.54(\mathrm{~s}, 3 \mathrm{H}), 0.89-1.22(\mathrm{~m}, 6 \mathrm{H}) ;{ }^{13} \mathrm{C} \mathrm{NMR}\left(125 \mathrm{MHz}, \mathrm{CDCl}_{3}\right) \delta$ $148.1,128.0,126.5,125.5,76.8,49.2,27.4,27.2,26.8,26.71,26.70,26.5$; IR (thin film) 
3449, 2956, 1602, 1446, 1165, $1028 \mathrm{~cm}^{-1}$; HRMS (ESI) $\mathrm{m} / \mathrm{z}$ calcd for $\mathrm{C}_{14} \mathrm{H}_{20} \mathrm{O}[\mathrm{M}+\mathrm{Na}]^{+}$ 227.1412, found 227.1401.

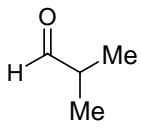

35

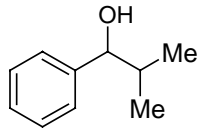

$( \pm)-36$

( \pm )-2-Methyl-1-phenylpropan-1-ol (36). The procedure for the synthesis of alcohol ( \pm )12 was followed using with 2-methylpropionaldehyde (35) (4.3 mL, $48 \mathrm{mmol}$ ), bromobenzene $(5.0 \mathrm{~mL}, 48 \mathrm{mmol}), n$-BuLi $(22 \mathrm{~mL}, 47 \mathrm{mmol}, 2.1 \mathrm{M}$ solution in hexane) to afford alcohol 36 as a clear oil $(3.7 \mathrm{~g}, 52 \%)$ after purification by flash chromatography (hexanes/EtOAc 19:1). Spectral data matched those reported in the literature: ${ }^{10}{ }^{1} \mathrm{H}$ NMR $\left(500 \mathrm{MHz}, \mathrm{CDCl}_{3}\right) \delta$ 7.23-7.33 (m, 5H), $4.31(\mathrm{~d}, J=6.9,1 \mathrm{H}), 2.05(\mathrm{~s}, 1 \mathrm{H}), 1.89-1.96(\mathrm{~m}$, $1 \mathrm{H}), 0.98(\mathrm{~d}, J=6.7,3 \mathrm{H}), 0.77(\mathrm{~d}, J=6.7,3 \mathrm{H}) ;{ }^{13} \mathrm{C} \mathrm{NMR}\left(125 \mathrm{MHz}, \mathrm{CDCl}_{3}\right) \delta 144.1$, $128.6,127.1,126.8,80.5,35.7,19.5,18.7$.

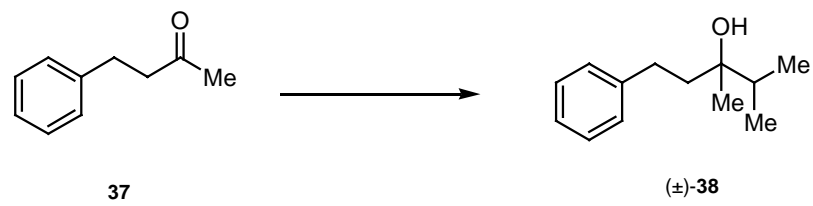

( \pm )-3,4-Dimethyl-1-phenyl-pentan-3-ol (38). To a cooled solution $\left(0{ }^{\circ} \mathrm{C}\right)$ of 4-phenylbutan-2-one (37) $(10.0 \mathrm{~mL}, 66.7 \mathrm{mmol})$ in $\mathrm{Et}_{2} \mathrm{O}(175 \mathrm{~mL})$ was added $i$-PrMgBr $(33 \mathrm{~mL}$, $66 \mathrm{mmol}, 2.0 \mathrm{M}$ solution in $\mathrm{Et}_{2} \mathrm{O}$ ) over one hour. The reaction mixture was stirred for two hours at ambient temperature and diluted with saturated aq $\mathrm{NH}_{4} \mathrm{Cl}(150 \mathrm{~mL})$ at $0{ }^{\circ} \mathrm{C}$. The aqueous layer was extracted with $\mathrm{Et}_{2} \mathrm{O}(75 \mathrm{~mL})$ and the combined organic layers were washed with brine $(100 \mathrm{~mL})$, dried over $\mathrm{MgSO}_{4}$, and concentrated in vacuo. The resulting oil was purified by flash chromatography (hexanes/EtOAc 19:1) yielding alcohol 38 (4.7 g, $24.5 \mathrm{mmol})$. Spectral data matched those reported in the literature: ${ }^{11}$ ${ }^{1} \mathrm{H}$ NMR $\left(500 \mathrm{MHz}, \mathrm{CDCl}_{3}\right) \delta$ 7.20-7.33 (m, 5H), 2.70-2.77 (m, 2H), 1.77-1.84 (m, 3H), $1.31(\mathrm{~s}, 1 \mathrm{H}), 1.22(\mathrm{~s}, 3 \mathrm{H}), 1.00(\mathrm{~d}, J=6.8,3 \mathrm{H}), 0.97(\mathrm{~d}, J=6.9,3 \mathrm{H}) ;{ }^{13} \mathrm{C} \mathrm{NMR}(125$ $\left.\mathrm{MHz}, \mathrm{CDCl}_{3}\right) \delta 143.1,128.7,128.6,126.0,74.9,42.1,37.3,30.2,23.3,17.8,17.2$; IR (thin film) 3419, 2877, 1603, 1497, 1374, $1094 \mathrm{~cm}^{-1}$; HRMS (ESI) $\mathrm{m} / \mathrm{z}$ calcd for $\mathrm{C}_{13} \mathrm{H}_{20} \mathrm{ONa}[\mathrm{M}+\mathrm{Na}]^{+}$215.1412, found 215.1411. Anal. Calcd for $\mathrm{C}_{13} \mathrm{H}_{20} \mathrm{O}: \mathrm{C}, 81.20 ; \mathrm{H}$, 10.48. Found: C, 81.16; H, 10.56 .

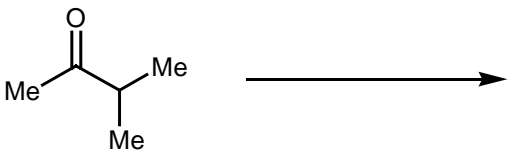

26

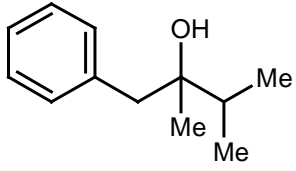

$( \pm)-39$

( \pm )-2,3-Dimethyl-1-phenylbutan-2-ol (39). ${ }^{\mathbf{1 2}}$ Benzyl chloride (23.7 mL, $\left.205 \mathrm{mmol}\right)$ was added to a slurry of magnesium $(5.00 \mathrm{~g}, 205 \mathrm{mmol})$ in refluxing $\mathrm{Et}_{2} \mathrm{O}(200 \mathrm{~mL})$ over one hour. The reaction mixture was stirred at reflux for two hours and 3-methylbutan-2-one 
(26) $(17.7 \mathrm{~mL}, 165 \mathrm{mmol})$ was added over one hour. The reaction mixture was stirred for 18 hours at ambient temperature and then was diluted with saturated aq $\mathrm{NH}_{4} \mathrm{Cl}(300 \mathrm{~mL})$. The organic layer was washed with saturated aq $\mathrm{NH}_{4} \mathrm{Cl}(300 \mathrm{~mL})$, brine $(100 \mathrm{~mL})$, dried over $\mathrm{MgSO}_{4}$, and concentrated in vacuo. The resulting oil was purified by distillation (bp $72-74{ }^{\circ} \mathrm{C}, 0.5 \mathrm{~mm} \mathrm{Hg}$ ) yielding alcohol 39 (12.3 g, 42\%). Spectral data matched those reported in the literature: ${ }^{12}{ }^{1} \mathrm{H}$ NMR $\left(500 \mathrm{MHz}, \mathrm{CDCl}_{3}\right) \delta 7.28-7.32(\mathrm{~m}, 2 \mathrm{H}), 7.21-7.25$ $(\mathrm{m}, 3 \mathrm{H}), 2.74(\mathrm{~d}, 13.3,1 \mathrm{H}), 2.70(\mathrm{~d}, 13.51 \mathrm{H}), 1.72(\mathrm{~m}, 1 \mathrm{H}), 1.26(\mathrm{~s}, 1 \mathrm{H}), 1.03(\mathrm{~s}, 3 \mathrm{H})$, $1.00(\mathrm{~d}, J=6.8,3 \mathrm{H}), 0.97(\mathrm{~d}, J=6.8,3 \mathrm{H}) ;{ }^{13} \mathrm{C} \mathrm{NMR}\left(125 \mathrm{MHz}, \mathrm{CDCl}_{3}\right) \delta 137.9,130.9$, 128.3, 126.6, 74.7, 45.3, 37.2, 23.0, 18.1, 17.4. Anal. Calcd for $\mathrm{C}_{12} \mathrm{H}_{18} \mathrm{O}: \mathrm{C}, 80.85 ; \mathrm{H}$, 10.18. Found: C, 80.69; H, 10.21.

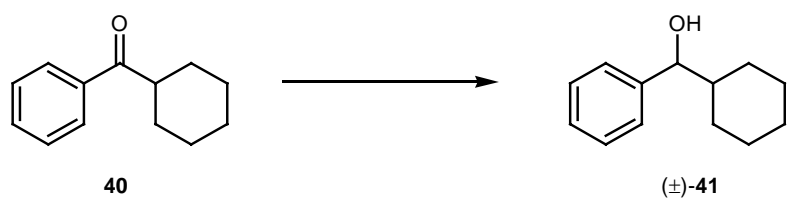

( \pm )-Cyclohexylphenylmethanol (41). To a slurry of cyclohexylphenylmethanone (40) $(3.5 \mathrm{~g}, 18 \mathrm{mmol})$ in $\mathrm{MeOH}(20 \mathrm{~mL})$ was added $\mathrm{NaBH}_{4}(0.70 \mathrm{~g}, 18 \mathrm{mmol})$. The slurry was stirred for ten minutes and then was diluted with $\mathrm{H}_{2} \mathrm{O}(15 \mathrm{~mL})$. The aqueous layer was extracted with $\mathrm{Et}_{2} \mathrm{O}(3 \times 20 \mathrm{~mL})$ and the combined organic layers were washed with brine $(100 \mathrm{~mL})$, dried over $\mathrm{MgSO}_{4}$, and concentrated in vacuo. The resulting oil was purified by flash chromatography (hexanes/EtOAc 19:1) yielding alcohol 41 (3.5 g, $73 \%)$. Spectral data matched those reported in the literature: ${ }^{13}{ }^{1} \mathrm{H}$ NMR $(500 \mathrm{MHz}$, $\left.\mathrm{CDCl}_{3}\right) \delta 7.32-7.42(\mathrm{~m}, 5 \mathrm{H}), 4.42(\mathrm{~d}, J=7.2,1 \mathrm{H}), 2.04-2.07(\mathrm{~m}, 1 \mathrm{H}), 1.95(\mathrm{~s}, 1 \mathrm{H}), 1.80-$ $1.87(\mathrm{~m}, 1 \mathrm{H}), 1.60-1.79(\mathrm{~m}, 3 \mathrm{H}), 1.42-1.46(\mathrm{~m}, 1 \mathrm{H}), 1.24-1.33(\mathrm{~m}, 5 \mathrm{H}) ;{ }^{13} \mathrm{C}$ NMR $(125$ $\left.\mathrm{MHz}_{2} \mathrm{CDCl}_{3}\right) \delta 143.8,128.4,127.6,126.8,79.6,45.1,29.5,29.0,26.6,26.3,26.2$; IR (thin film) 3409, 2852, 1603, 1493, 1451, $1015 \mathrm{~cm}^{-1}$; HRMS (ESI) $\mathrm{m} / \mathrm{z}$ calcd for $\mathrm{C}_{13} \mathrm{H}_{18} \mathrm{ONa}[\mathrm{M}+\mathrm{Na}]^{+}$213.1255, found 213.1259. Anal. Calcd for $\mathrm{C}_{13} \mathrm{H}_{18} \mathrm{O}: \mathrm{C}, 82.06 ; \mathrm{H}$, 9.53. Found: C, 82.08; H, 9.53.

\section{Preparation of Hydroperoxides 11, 13-24}

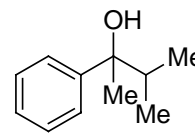

$( \pm)-12$

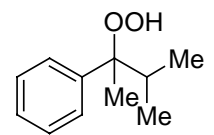

$( \pm)-11$

( \pm )-3-Methyl-2-phenylbut-2-yl hydroperoxide (11). ${ }^{14}$ To a cooled $\left(0{ }^{\circ} \mathrm{C}\right)$ solution of $\mathrm{H}_{2} \mathrm{O}_{2}\left(65 \mathrm{ml}, 1.1 \mathrm{~mol}, 50 \%\right.$ wt in $\left.\mathrm{H}_{2} \mathrm{O}\right)$ and $\mathrm{H}_{2} \mathrm{SO}_{4}(1.0 \mathrm{~mL}, 19 \mathrm{mmol})$ was added 3methyl-2-phenylbutan-2-ol (12) (6.9 g, $42 \mathrm{mmol})$. The reaction mixture was stirred vigorously at ambient temperature for 14 hours and then was partitioned between $\mathrm{Et}_{2} \mathrm{O}$ $(100 \mathrm{~mL})$ and water $(100 \mathrm{~mL})$. The aqueous layer was extracted with $\mathrm{Et}_{2} \mathrm{O}(2 \times 50 \mathrm{~mL})$ and the combined organic layers were washed with $1 \mathrm{~N} \mathrm{NaOH}(3 \times 25 \mathrm{~mL})$ and brine $(50$ $\mathrm{mL}$ ). The resulting solution was dried over $\mathrm{MgSO}_{4}$ and concentrated in vacuo to yield hydroperoxide $11(6.1 \mathrm{~g}, 78 \%)$ as a clear oil. Spectral data matched those reported in the literature: ${ }^{15}{ }^{1} \mathrm{H}$ NMR $\left(500 \mathrm{MHz}, \mathrm{CDCl}_{3}\right) \delta 7.42-7.48(\mathrm{~m}, 4 \mathrm{H}), 7.37(\mathrm{tt}, J=6.8,1.8,1 \mathrm{H})$, 
$7.32(\mathrm{~s}, 1 \mathrm{H}), 2.00(\mathrm{sp}, J=6.9,1 \mathrm{H}), 1.66(\mathrm{~s}, 3 \mathrm{H}), 0.96(\mathrm{~d}, J=6.8,3 \mathrm{H}), 0.69(\mathrm{~d}, J=6.9$, $3 \mathrm{H}) ;{ }^{13} \mathrm{C}$ NMR $\left(125 \mathrm{MHz}, \mathrm{CDCl}_{3}\right) \delta 143.3,128.3,127.2,126.3,89.1,37.0,17.9,17.4$, 17.3; IR (thin film) 3422, 2965, 2878, $1446,1030 \mathrm{~cm}^{-1}$. Anal. Calcd for $\mathrm{C}_{11} \mathrm{H}_{16} \mathrm{O}_{2}$ : C, 73.30; H, 8.95. Found: C, 73.04; H, 8.93.

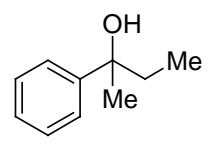

$( \pm)-42$

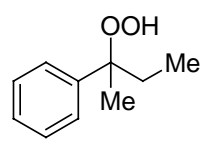

$( \pm)-13$

(士)-2-Phenylbut-2-yl hydroperoxide (13). The procedure for the synthesis of hydroperoxide $( \pm)$-11 was followed using alcohol $42(5.0 \mathrm{~g}, 33.3 \mathrm{mmol})$ to afford hydroperoxide 13 as a clear oil $(4.2 \mathrm{~g}, 76 \%)$ after purification by flash chromatography (hexanes/EtOAc 19:1). Previous literature report does not provide NMR spectral data: ${ }^{16}$ ${ }^{1} \mathrm{H}$ NMR $\left(500 \mathrm{MHz}, \mathrm{CDCl}_{3}\right) \delta 7.54(\mathrm{~s}, 1 \mathrm{H}), 7.36-7.48(\mathrm{~m}, 4 \mathrm{H}), 7.29-7.35(\mathrm{~m}, 1 \mathrm{H}), 1.85-$ $1.92(\mathrm{~m}, 2 \mathrm{H}), 1.68(\mathrm{~s}, 3 \mathrm{H}), 0.87(\mathrm{t}, J=7.4,3 \mathrm{H}) ;{ }^{13} \mathrm{C} \mathrm{NMR}\left(125 \mathrm{MHz}, \mathrm{CDCl}_{3}\right) \delta 144.0$, 128.6, 127.5, 125.9, 86.9, 32.5, 22.1, 8.5. HRMS (ESI) $m / z$ calcd for $\mathrm{C}_{10} \mathrm{H}_{14} \mathrm{O}_{2} \mathrm{Na}$ $[\mathrm{M}+\mathrm{Na}]^{+}$189.0892, found 189.0897 .

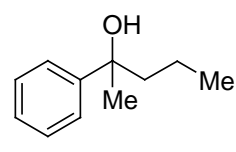

$( \pm)-28$

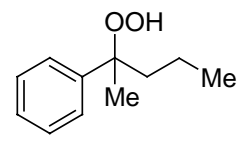

$( \pm)-14$

(士)-2-Phenylpent-2-yl hydroperoxide (14). The procedure for the synthesis of hydroperoxide $( \pm)$-11 was followed using alcohol $28(1.0 \mathrm{~mL}, 5.9 \mathrm{mmol})$ to afford hydroperoxide 14 as a clear oil $(0.48 \mathrm{~g}, 46 \%)$ after purification by flash chromatography (hexanes/EtOAc 19:1). Spectral data matched those reported in the literature: ${ }^{17}{ }^{1} \mathrm{H}$ NMR $\left(500 \mathrm{MHz}, \mathrm{CDCl}_{3}\right) \delta$ 7.38-7.45 (m, 4H), 7.29-7.32 (m, 1H), 7.21 (s, 1H), 1.75-1.80 (m, $2 \mathrm{H}), 1.67(\mathrm{~s}, 3 \mathrm{H}), 1.30-1.35(\mathrm{~m}, 1 \mathrm{H}), 1.18-1.20(\mathrm{~m}, 1 \mathrm{H}), 0.88(\mathrm{t}, J=7.4,3 \mathrm{H}) ;{ }^{13} \mathrm{C} \mathrm{NMR}$ $\left(125 \mathrm{MHz}, \mathrm{CDCl}_{3}\right) \delta 144.1,128.6,127.4,125.7,86.6,42.2,22.5,17.3,14.5$; IR (thin film) 3427, 2960, 2873, 1447, 1314, $1028 \mathrm{~cm}^{-1}$. Anal. Calcd for $\mathrm{C}_{11} \mathrm{H}_{16} \mathrm{O}: \mathrm{C}, 73.30 ; \mathrm{H}$, 8.95. Found: C, 73.26; H, 9.07.

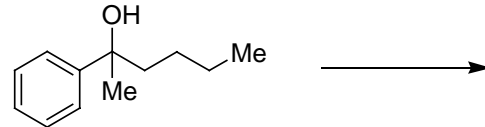

$( \pm)-30$

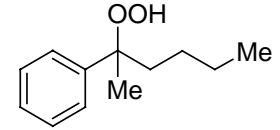

$( \pm)-15$

( \pm )-2-Phenylhex-2-yl hydroperoxide (15). The procedure for the synthesis of hydroperoxide $( \pm)$-11 was followed using alcohol $30(1.0 \mathrm{~g}, 5.6 \mathrm{mmol})$ to afford hydroperoxide 15 as a clear oil $(0.70 \mathrm{~g}, 65 \%)$ after purification by flash chromatography (hexanes/EtOAc 19:1). Spectral data matched those reported in the literature: ${ }^{18}{ }^{1} \mathrm{H}$ NMR $\left(500 \mathrm{MHz}, \mathrm{CDCl}_{3}\right) \delta$ 7.45-7.46 (m, 2H), 7.39-7.44 (m, 2H), 7.27-7.33 (m, 1H), 7.28 (s, $1 \mathrm{H}), 1.75-1.82(\mathrm{~m}, 2 \mathrm{H}), 1.68(\mathrm{~s}, 3 \mathrm{H}), 1.24-1.31(\mathrm{~m}, 3 \mathrm{H}), 1.11-1.16(\mathrm{~m}, 1 \mathrm{H}), 0.88(\mathrm{t}, J=$ 7.3, 3H); ${ }^{13} \mathrm{C}$ NMR $\left(125 \mathrm{MHz}, \mathrm{CDCl}_{3}\right) \delta 144.3,128.7,127.5,125.9,86.7,39.8,26.2$, 23.3, 22.6, 14.2. Anal. Calcd for $\mathrm{C}_{12} \mathrm{H}_{18} \mathrm{O}_{2}$ : C, 74.19; H, 9.34. Found: C, 74.02; H, 9.43. 


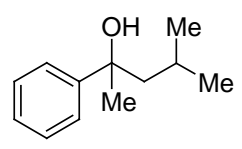

$( \pm)-32$

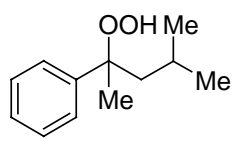

(士)-16

( \pm )-4-Methyl-2-phenylpent-2-yl hydroperoxide (16). The procedure for the synthesis of hydroperoxide $( \pm)-11$ was followed using alcohol $32(3.2 \mathrm{~mL}, 5.9 \mathrm{mmol})$ to afford hydroperoxide 16 as a clear oil $(1.9 \mathrm{~g}, 57 \%)$ after purification by flash chromatography (hexanes/EtOAc 19:1). Spectral data matched those reported in the literature: ${ }^{8}{ }^{1} \mathrm{H}$ NMR $\left(500 \mathrm{MHz}, \mathrm{CDCl}_{3}\right) \delta$ 7.38-7.47 (m, 4H), 7.29-7.32 (m, 1H), $7.29(\mathrm{~s}, 1 \mathrm{H}), 1.65-1.74(\mathrm{~m}$, $6 \mathrm{H}), 0.90(\mathrm{~d}, J=6.6,3 \mathrm{H}), 0.78(\mathrm{~d}, J=6.6,3 \mathrm{H}) ;{ }^{13} \mathrm{C} \mathrm{NMR}\left(125 \mathrm{MHz}, \mathrm{CDCl}_{3}\right) \delta 144.5$, $128.5,127.3,125.8,87.0,49.0,24.5,24.4,24.3,22.8$; IR (thin film) 3418, 2950, 1951, $1603,1447,1029 \mathrm{~cm}^{-1}$. Anal. Calcd for $\mathrm{C}_{12} \mathrm{H}_{18} \mathrm{O}_{2}: \mathrm{C}, 74.19 ; \mathrm{H}, 9.34$. Found: $\mathrm{C}, 74.12 ; \mathrm{H}$, 9.36 .

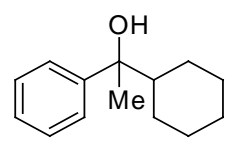

$( \pm)-34$

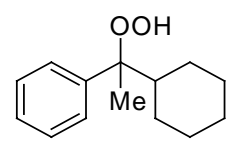

$( \pm)-17$

( \pm )-1-Cyclohexyl-1-phenyleth-1-yl hydroperoxide (17). The procedure for the synthesis of hydroperoxide $( \pm)$-11 was followed using alcohol $\mathbf{3 4}(1.0 \mathrm{~g}, 4.9 \mathrm{mmol})$ to afford hydroperoxide 17 as a clear oil $(0.5 \mathrm{~g}, 42 \%)$ after purification by flash chromatography (hexanes/EtOAc 19:1). Spectral data matched those reported in the literature: ${ }^{17}{ }^{1} \mathrm{H}$ NMR $\left(500 \mathrm{MHz}, \mathrm{CDCl}_{3}\right) \delta$ 7.39-7.40 (m, 4H), 7.30-7.32 (m, $\left.1 \mathrm{H}\right), 7.09(\mathrm{~s}$, $1 \mathrm{H}), 1.60-1.69(\mathrm{~m}, 8 \mathrm{H}), 0.91-1.09(\mathrm{~m}, 6 \mathrm{H}) ;{ }^{13} \mathrm{C} \mathrm{NMR}\left(125 \mathrm{MHz}, \mathrm{CDCl}_{3}\right) \delta 143.7,128.5$, 127.4, 126.5, 89.7, 47.8, 28.0, 27.8, 26.8, 26.7, 26.6, 18.6; IR (thin film) 3419, 2929, $1603,1447,1059,1028 \mathrm{~cm}^{-1}$. Anal. Calcd for $\mathrm{C}_{14} \mathrm{H}_{20} \mathrm{O}_{2}: \mathrm{C}, 76.33 ; \mathrm{H}, 9.15$. Found: $\mathrm{C}$, $76.60 ; \mathrm{H}, 9.22$.

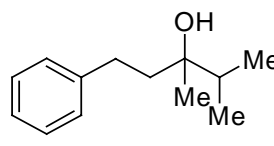

$( \pm)-38$

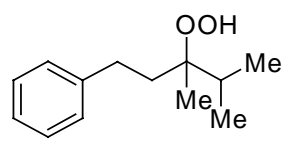

$( \pm)-24$

( \pm )-3,4-Dimethyl-1-phenylpent-3-yl hydroperoxide (24). The procedure for the synthesis of hydroperoxide $( \pm)-\mathbf{1 1}$ was followed using alcohol $\mathbf{3 8}(1.0 \mathrm{~g}, 4.9 \mathrm{mmol})$ to afford hydroperoxide 24 as a clear oil $(0.30 \mathrm{~g}, 30 \%)$ after purification by flash chromatography (hexanes/EtOAc 19:1) as a clear oil (0.30 g, 30\%): ${ }^{1} \mathrm{H}$ NMR (500 MHz, $\left.\mathrm{CDCl}_{3}\right) \delta$ 7.31-7.33 (m, 2H), 7.24-7.30 (m, 2H), 7.19-7.23 (m, 1H), $9.98(\mathrm{~s}, 1 \mathrm{H}), 2.69-$ $2.73(\mathrm{~m}, 2 \mathrm{H}), 2.10-2.15(\mathrm{~m}, 1 \mathrm{H}), 1.90-1.94(\mathrm{~m}, 2 \mathrm{H}), 1.16(\mathrm{~s}, 3 \mathrm{H}), 0.93-0.97(\mathrm{~m}, 6 \mathrm{H}) ;{ }^{13} \mathrm{C}$ NMR (125 MHz, $\left.\mathrm{CDCl}_{3}\right) \delta 143.2,128.6,128.5,126.0,87.0,37.0,32.8,29.8,17.9,17.2$, 17.1; IR (thin film) 3406, 2962, 1603, 1497, 1096, $1030 \mathrm{~cm}^{-1}$. 


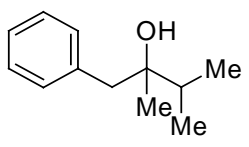

$( \pm)-39$

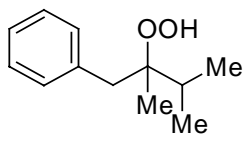

$( \pm)-23$

( \pm )-2,3-Dimethyl-1-phenylbut-2-yl hydroperoxide (23). ${ }^{\mathbf{1 9}}$ To a solution of $\mathrm{H}_{2} \mathrm{O}_{2}(20.0$ $\mathrm{mL}, 320 \mathrm{mmol}, 50 \%$ wt in $\left.\mathrm{H}_{2} \mathrm{O}\right)$ and $\mathrm{H}_{3} \mathrm{PO}_{4}(8.0 \mathrm{~mL}, 18 \mathrm{mmol})$ was added alcohol 39 $(1.0 \mathrm{~g}, 4.9 \mathrm{mmol})$. The reaction mixture was heated $\left(40{ }^{\circ} \mathrm{C}\right)$ for 18 hours and then was partitioned between $\mathrm{Et}_{2} \mathrm{O}(50 \mathrm{~mL})$ and $\mathrm{H}_{2} \mathrm{O}(50 \mathrm{~mL})$. The aqueous layer was extracted with $\mathrm{Et}_{2} \mathrm{O}(2 \times 25 \mathrm{~mL})$ and the combined organic layers were washed with brine $(50 \mathrm{~mL})$, dried over $\mathrm{MgSO}_{4}$, and concentrated in vacuo. Purification by flash chromatography (hexanes/EtOAc 5:1) yielded hydroperoxide 23 as a clear oil $(0.31 \mathrm{~g}, 31 \%):{ }^{1} \mathrm{H}$ NMR $\left(500 \mathrm{MHz}, \mathrm{CDCl}_{3}\right) \delta 7.22-7.32(\mathrm{~m}, 5 \mathrm{H}), 7.08(\mathrm{~s}, 1 \mathrm{H}), 2.95(\mathrm{~d}, J=14.2,1 \mathrm{H}), 2.85(\mathrm{~d}, J=$ $14.2,1 \mathrm{H}), 2.06-2.12(\mathrm{~m}, 1 \mathrm{H}), 1.03(\mathrm{~s}, 3 \mathrm{H}), 1.01(\mathrm{~d}, J=6.9,3 \mathrm{H}), 0.97(\mathrm{~d}, J=6.9,3 \mathrm{H})$;

${ }^{13} \mathrm{C}$ NMR $\left(125 \mathrm{MHz}, \mathrm{CDCl}_{3}\right) \delta 137.9,130.8,128.2,126.3,87.4,40.1,32.9,18.1,17.4$, 17.2.

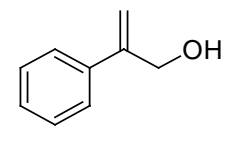

43

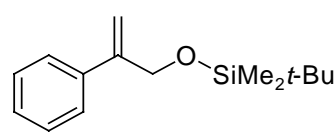

44

tert-Butyl-dimethyl-(2-phenyl-allyloxy)-silane (44). To a cooled $\left(0{ }^{\circ} \mathrm{C}\right)$ solution of alcohol $43^{20}(2.6 \mathrm{~g}, 20 \mathrm{mmol})$ in $\mathrm{CH}_{2} \mathrm{Cl}_{2}(40 \mathrm{~mL})$ were added $t-\mathrm{BuMe}{ }_{2} \mathrm{SiCl}(3.8 \mathrm{~g}, 25$ $\mathrm{mmol})$ and $\mathrm{Et}_{3} \mathrm{~N}(8.2 \mathrm{~mL}, 59 \mathrm{mmol})$ over one half hour. The reaction mixture was stirred at ambient temperature for 12 hours and then partitioned between water $(100 \mathrm{~mL})$ and $\mathrm{CH}_{2} \mathrm{Cl}_{2}(100 \mathrm{~mL})$. The organic layer was washed with brine $(50 \mathrm{~mL})$, dried over $\mathrm{MgSO}_{4}$ and concentrated in vacuo. The resulting oil was purified by flash chromatography (hexanes/EtOAc 50:1) to yield tert-butyl-dimethyl-(2-phenyl-allyloxy)-silane $\mathbf{4 4}$ as a clear oil $(4.1 \mathrm{~g}, 84 \%):{ }^{1} \mathrm{H}$ NMR $\left(500 \mathrm{MHz}, \mathrm{CDCl}_{3}\right) \delta$ 7.42-7.48 (m, 2H), 7.35-7.41 (m, $2 \mathrm{H}), 7.30-7.34(\mathrm{~m}, 1 \mathrm{H}), 5.47(\mathrm{~d}, J=10.8 \mathrm{~Hz}, 2 \mathrm{H}), 4.58(\mathrm{~s}, 2 \mathrm{H}), 0.99$ (s, 9H), 0.16 (s, $6 \mathrm{H}) ;{ }^{13} \mathrm{C}$ NMR $\left(125 \mathrm{MHz}, \mathrm{CDCl}_{3}\right) \delta 147.2,139.4,128.5,127.8,126.2,111.5,64.9,26.1$, 18.6, -5.1; IR (thin film) 3245, 3033, 2858, 1254, 1129, $1082 \mathrm{~cm}^{-1}$.

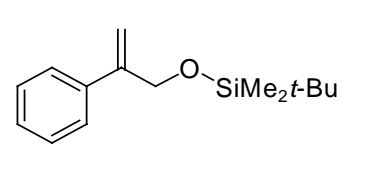

44

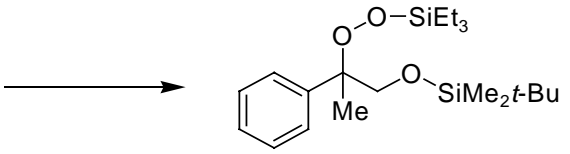

45

1-(tert-Butyl-dimethyl-silanyloxy)-2-phenyl-prop-2-yl-hydroperoxytriethylsilyl ether (45). To a solution of silyl ether $\mathbf{4 4}(2.3 \mathrm{~g}, 9.4 \mathrm{mmol})$ in anhydrous EtOH $(20 \mathrm{~mL})$ were added $\mathrm{Co}(\mathrm{acac})_{2}(0.24 \mathrm{~g}, 0.94 \mathrm{mmol})$ and $\mathrm{Et}_{3} \mathrm{SiH}(3.0 \mathrm{~mL}, 18.8 \mathrm{mmol})$. The reaction mixture was stirred under $\mathrm{O}_{2}(1 \mathrm{~atm})$ for one half hour and then was concentrated in vacuo. The resulting dark green oil was partitioned between $\mathrm{H}_{2} \mathrm{O}(50 \mathrm{~mL})$ and EtOAc $(50 \mathrm{~mL})$ and the aqueous layer was extracted with EtOAc $(2 \times 25 \mathrm{~mL})$. The organic layers were combined and washed with brine $(50 \mathrm{~mL})$, dried with $\mathrm{MgSO}_{4}$ and 
concentrated in vacuo. Purification by flash chromatography (hexanes/EtOAc 33:1) afforded 1-(tert-butyl-dimethyl-silanyloxy)-2-phenyl-prop-2-yl-hydroperoxytriethylsilyl ether (45) as a clear oil (1.8 g, 49\%): ${ }^{1} \mathrm{H}$ NMR (500 MHz, $\left.\mathrm{CDCl}_{3}\right) \delta 7.44-7.48(\mathrm{~m}, 2 \mathrm{H})$, 7.30-7.35 (m, 2H), 7.23-7.28 (m, 1H), $3.78(\mathrm{~d}, J=10.4,1 \mathrm{H}), 3.74(\mathrm{~d}, J=10.4,1 \mathrm{H}), 1.64$ $(\mathrm{s}, 3 \mathrm{H}), 1.00(\mathrm{t}, J=7.8,9 \mathrm{H}), 0.86(\mathrm{~s}, 9 \mathrm{H}), 0.72(\mathrm{q}, J=7.7,6 \mathrm{H}),-0.02(\mathrm{~s}, 3 \mathrm{H}),-0.05(\mathrm{~s}$, $3 \mathrm{H}) ;{ }^{13} \mathrm{C}$ NMR $\left(125 \mathrm{MHz}, \mathrm{CDCl}_{3}\right) \delta 143.3,127.9,127.2,126.6,86.4,68.6,26.0,20.4$, 18.4, 7.0, 4.0, -5.4, -5.5; IR (thin film) 2956, 2931, 1727, 1472, 1252, $1109 \mathrm{~cm}^{-1}$. HRMS (ESI) $\mathrm{m} / \mathrm{z}$ calcd for $\mathrm{C}_{21} \mathrm{H}_{40} \mathrm{O}_{3} \mathrm{Si}_{2} \mathrm{Na}[\mathrm{M}+\mathrm{Na}]^{+} 419.2414$, found 419.2415.

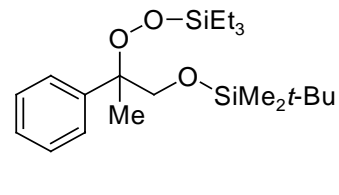

45

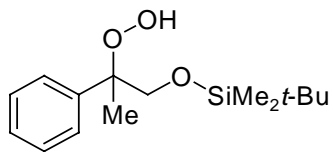

18

1-(tert-Butyl-dimethyl-silanyloxy)-2-phenyl-prop-2-yl-hydroperoxide (18). A solution of silylperoxy ether (45) $(0.50 \mathrm{~g}, 1.3 \mathrm{mmol})$ in $2 \mathrm{M} \mathrm{NaOH} / \mathrm{MeOH}(5: 95)$ was stirred at ambient temperature for 45 minutes. The reaction mixture was then diluted with $\mathrm{H}_{2} \mathrm{O}(20$ $\mathrm{mL})$ and extracted with EtOAc $(2 \times 20 \mathrm{~mL})$. The combined organic layers were washed with brine $(20 \mathrm{~mL})$, dried over $\mathrm{MgSO}_{4}$, and concentrated in vacuo. Purification by flash chromatography (hexanes/EtOAc 9:1) yielded 1-(tert-butyl-dimethyl-silanyloxy)-2phenyl-prop-2-yl-hydroperoxide (18) as a clear oil $(0.06 \mathrm{~g}, 17 \%)$ : ${ }^{1} \mathrm{H}$ NMR $(500 \mathrm{MHz}$, $\left.\mathrm{CDCl}_{3}\right) \delta 8.15(\mathrm{~s}, 1 \mathrm{H}), 7.47-7.51(\mathrm{~m}, 2 \mathrm{H}), 7.37-7.41(\mathrm{~m}, 2 \mathrm{H}), 7.30-7.33(\mathrm{~m}, 1 \mathrm{H}), 3.94(\mathrm{~d}$, $J=10.6,1 \mathrm{H}), 3.88(\mathrm{~d}, J=10.6,1 \mathrm{H}), 1.65(\mathrm{~s}, 3 \mathrm{H}), 0.91(\mathrm{~s}, 9 \mathrm{H}), 0.06(\mathrm{~s}, 3 \mathrm{H}), 0.04(\mathrm{~s}$, $3 \mathrm{H}) ;{ }^{13} \mathrm{C}$ NMR $\left(125 \mathrm{MHz}, \mathrm{CDCl}_{3}\right) \delta 142.1,128.5,127.8,126.2,86.3,68.9,26.0,21.2$, 18.5, -5.4, -5.5; IR (thin film) 3417, 2931, 1472, 1447, 1254, $1111 \mathrm{~cm}^{-1}$. HRMS (ESI) $\mathrm{m} /$ $Z$ calcd for $\mathrm{C}_{15} \mathrm{H}_{26} \mathrm{O}_{3} \mathrm{SiNa}[\mathrm{M}+\mathrm{Na}]^{+}$305.1549, found 305.1536. Anal. Calcd for $\mathrm{C}_{15} \mathrm{H}_{26} \mathrm{O}_{3} \mathrm{Si}: \mathrm{C}, 63.78 ; \mathrm{H}, 9.28$. Found: C, 64.03; H, 9.38.

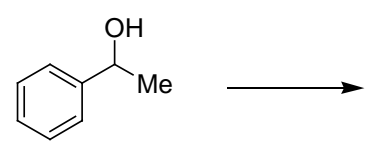

$( \pm)-46$

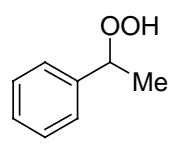

$( \pm)-19$

(士)-1-Phenyleth-1-yl hydroperoxide (19). ${ }^{21}$ To a cooled $\left(0{ }^{\circ} \mathrm{C}\right)$ solution of $\mathrm{H}_{2} \mathrm{O}_{2}(30 \mathrm{ml}$, $0.50 \mathrm{~mol}, 50 \% \mathrm{wt}$ in $\left.\mathrm{H}_{2} \mathrm{O}\right)$ and $\mathrm{H}_{2} \mathrm{SO}_{4}(0.5 \mathrm{~mL}, 9 \mathrm{mmol})$ was added 1-phenylethanol (46) $(2.3 \mathrm{~g}, 18 \mathrm{mmol})$. The reaction mixture was stirred vigorously for 72 hours at ambient temperature and partitioned between $\mathrm{Et}_{2} \mathrm{O}(20 \mathrm{~mL})$ and water $(30 \mathrm{~mL})$. The aqueous layer was extracted with $\mathrm{Et}_{2} \mathrm{O}(2 \times 20 \mathrm{~mL})$ and the combined organic layers were washed with $1 \mathrm{~N} \mathrm{aq} \mathrm{NaOH}(2 \times 10 \mathrm{~mL})$ and brine $(20 \mathrm{~mL})$. The resulting solution was dried over $\mathrm{MgSO}_{4}$, concentrated in vacuo and was purified by flash chromatography (hexanes/EtOAc 9:1) to yield hydroperoxide $19(1.7 \mathrm{~g}, 76 \%)$. Spectral data matched those reported in literature: ${ }^{22}{ }^{1} \mathrm{H}$ NMR $\left(500 \mathrm{MHz}, \mathrm{CDCl}_{3}\right) \delta 8.03(\mathrm{~s}, 1 \mathrm{H}), 7.38-7.47(\mathrm{~m}$, $5 \mathrm{H}), 5.13(\mathrm{q}, J=6.6,1 \mathrm{H}), 1.54(\mathrm{~d}, J=6.6,3 \mathrm{H}) ;{ }^{13} \mathrm{C} \mathrm{NMR}\left(125 \mathrm{MHz}, \mathrm{CDCl}_{3}\right) \delta 141.9$, 129.1, 128.7, 127.0, 84.2, 20.5. HRMS (ESI) $\mathrm{m} / \mathrm{z}$ calcd for $\mathrm{C}_{8} \mathrm{H}_{10} \mathrm{O}_{2} \mathrm{Na}[\mathrm{M}+\mathrm{Na}]^{+}$ 161.0578 , found 161.0585 . 


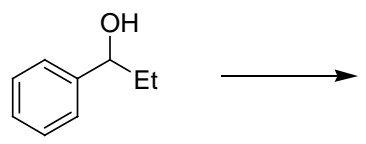

$( \pm)-47$

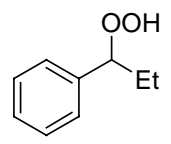

$( \pm)-20$

(士)-1-Cyclohexyl-1-phenyleth-1-yl hydroperoxide (20). The procedure for the synthesis of hydroperoxide $( \pm)-\mathbf{1 9}$ was followed using alcohol 47 (2.2 g, $16 \mathrm{mmol})$ to afford hydroperoxide 20 as a clear oil (1.3 g, 52\%) after purification by flash chromatography (hexanes/EtOAc 19:1). Previous literature report does not provide NMR spectral data. ${ }^{23}{ }^{1} \mathrm{H}$ NMR $\left(500 \mathrm{MHz}, \mathrm{CDCl}_{3}\right) \delta 7.92(\mathrm{~s}, 1 \mathrm{H}), 7.32-7.42(\mathrm{~m}, 5 \mathrm{H}), 4.83(\mathrm{t}, J$ $=6.9,1 \mathrm{H}), 1.87-1.96(\mathrm{~m}, 1 \mathrm{H}), 1.65-1.74(\mathrm{~m}, 1 \mathrm{H}), 0.92(\mathrm{t}, J=7.5,3 \mathrm{H}) ;{ }^{13} \mathrm{C} \mathrm{NMR}(125$ $\left.\mathrm{MHz}, \mathrm{CDCl}_{3}\right) \delta 141.0,129.0,128.6,127.4,90.1,27.9,10.6$. HRMS (ESI) $\mathrm{m} / \mathrm{z}$ calcd for $\mathrm{C}_{9} \mathrm{H}_{12} \mathrm{O}_{2} \mathrm{Na}[\mathrm{M}+\mathrm{Na}]^{+}$175.0735, found 175.0739.

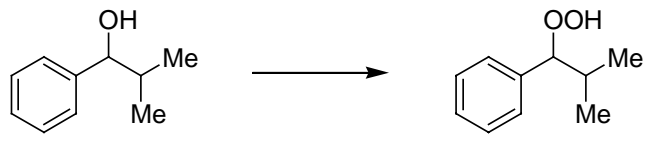

$( \pm)-38$

$( \pm)-21$

(士)-2-Methyl-1-phenylprop-1-yl hydroperoxide (21). The procedure for the synthesis of hydroperoxide $( \pm)$-19 was followed using alcohol $38(1.0 \mathrm{~g}, 16 \mathrm{mmol})$ to afford hydroperoxide 21 as a clear oil $(0.50 \mathrm{~g}, 44 \%)$ after purification by flash chromatography (hexanes/EtOAc 19:1). Previous literature report does not provide NMR spectral data. ${ }^{23}$ ${ }^{1} \mathrm{H}$ NMR $\left(400 \mathrm{MHz}, \mathrm{CDCl}_{3}\right) \delta 7.90(\mathrm{~s}, 1 \mathrm{H}), 7.26-7.36(\mathrm{~m}, 5 \mathrm{H}), 4.55(\mathrm{~d}, J=8,1 \mathrm{H}), 1.93-$ $1.99(\mathrm{~m}, 1 \mathrm{H}), 1.03(\mathrm{~d}, J=6.7,3 \mathrm{H}), 0.92(\mathrm{~d}, J=6.7,3 \mathrm{H}) ;{ }^{13} \mathrm{C} \mathrm{NMR}\left(100 \mathrm{MHz}, \mathrm{CDCl}_{3}\right) \delta$ 149.0, 128.5, 128.1, 127.5, 93.7, 32.5, 19.4, 19.1. HRMS (ESI) $m / z$ calcd for $\mathrm{C}_{10} \mathrm{H}_{14} \mathrm{O}_{2} \mathrm{Na}[\mathrm{M}+\mathrm{Na}]^{+}$189.0892, found 189.0899 .

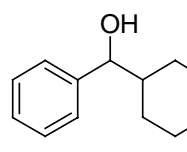

$( \pm)-41$

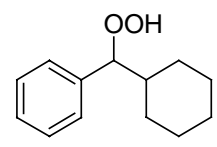

$( \pm)-22$

( \pm )-Cyclohexylphenylmethyl hydroperoxide (22). The procedure for the synthesis of hydroperoxide $( \pm)$-19 was followed using alcohol $41(0.90 \mathrm{~g}, 16 \mathrm{mmol})$ to afford hydroperoxide 22 as a clear oil $(0.30 \mathrm{~g}, 33 \%)$ after purification by flash chromatography (hexanes/EtOAc 19:1). Previous literature report does not provide NMR spectral data. ${ }^{24}$ ${ }^{1} \mathrm{H}$ NMR $\left(400 \mathrm{MHz}, \mathrm{CDCl}_{3}\right) \delta 7.72(\mathrm{~s}, 1 \mathrm{H}), 7.30-7.42(\mathrm{~m}, 5 \mathrm{H}), 4.66(\mathrm{~d}, J=8.1,1 \mathrm{H})$, 2.04-2.08 (m, 1H), 1.61-1.78 (m, 4H), 0.91-1.34 (m, 6H); ${ }^{13} \mathrm{C}$ NMR (100 MHz, $\left.\mathrm{CDCl}_{3}\right) \delta$ $140.0,128.6,128.2,127.5,93.0,42.2,29.8,29.4,26.5,26.2,26.0$. HRMS (ESI) $\mathrm{m} / \mathrm{z}$ calcd for $\mathrm{C}_{13} \mathrm{H}_{18} \mathrm{O}_{2} \mathrm{Na}[\mathrm{M}+\mathrm{Na}]^{+} 229.1205$, found 229.1200 .

\section{Preparation of Xylyl-PHANEPHOS 11}




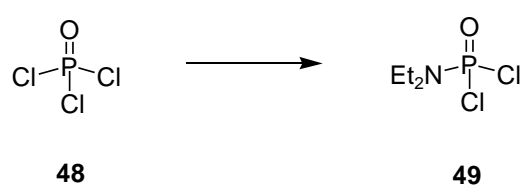

$\boldsymbol{N}, \boldsymbol{N}$-Diethylphosphoroamidic dichloride (49). ${ }^{25}$ To a cooled $\left(0{ }^{\circ} \mathrm{C}\right)$ solution of $\mathrm{POCl}_{3}$ (48) $(3.7 \mathrm{~mL}, 40 \mathrm{mmol})$ and $\mathrm{Et}_{3} \mathrm{~N}(6.3 \mathrm{~mL}, 45 \mathrm{mmol})$ in $\mathrm{CH}_{2} \mathrm{Cl}_{2}(150 \mathrm{~mL})$ was added $\mathrm{Et}_{2} \mathrm{NH}(4.1 \mathrm{~mL}, 40 \mathrm{mmol})$ over one hour. The reaction mixture was stirred at ambient temperature for 24 hours and then was diluted with $10 \%$ aq $\mathrm{KH}_{2} \mathrm{PO}_{4}(50 \mathrm{~mL})$. The resulting mixture was extracted with $\mathrm{CH}_{2} \mathrm{Cl}_{2}(2 \times 50 \mathrm{~mL})$ and the combined organic layers were washed with brine $(50 \mathrm{~mL})$ and concentrated in vacuo. Purification by distillation (bp: $62-64{ }^{\circ} \mathrm{C}$; $0.5 \mathrm{~mm} \mathrm{Hg}$ ) yielded compound 49 as a clear oil $(6.1 \mathrm{~g}, 81 \%)$. Spectral data matched those reported in literature: ${ }^{25}{ }^{1} \mathrm{H} \mathrm{NMR}\left(500 \mathrm{MHz}, \mathrm{CDCl}_{3}\right) \delta 3.27$ $3.35(\mathrm{~m}, 4 \mathrm{H}), 1.20-1.26(\mathrm{~m}, 6 \mathrm{H}) ;{ }^{13} \mathrm{C} \mathrm{NMR}\left(125 \mathrm{MHz}, \mathrm{CDCl}_{3}\right) \delta 40.9,13.4 ;{ }^{31} \mathrm{P} \mathrm{NMR}$ $\left(162 \mathrm{MHz}, \mathrm{CDCl}_{3}\right) \delta$ 14; HRMS (ESI) $m / z$ calcd for $\mathrm{C}_{4} \mathrm{H}_{10} \mathrm{ONPCl}_{2} \mathrm{Na}[\mathrm{M}+\mathrm{Na}]^{+}$ 211.9775, found 211.9769.

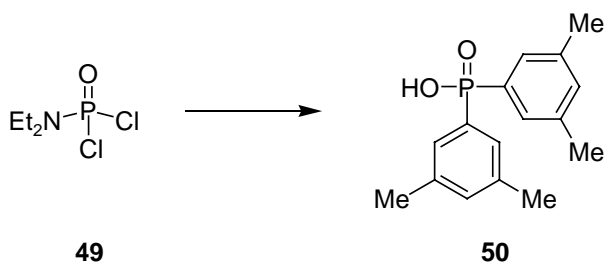

Bis(3,5-dimethylphenyl)phosphinic acid (50). To a cooled $\left(-78{ }^{\circ} \mathrm{C}\right)$ solution of $3,5-$ dimethylbromobenzene $(10.0 \mathrm{~mL}, 73.5 \mathrm{mmol})$ in THF $(100 \mathrm{~mL})$ was added $n$-BuLi (37 $\mathrm{mL}, 77 \mathrm{mmol}, 2.1 \mathrm{M}$ solution in hexane) over one hour. The reaction mixture was stirred at ambient temperature for one-half hour then cooled $\left(-78^{\circ} \mathrm{C}\right)$. Electrophile $49(5.6 \mathrm{~mL}$, $37 \mathrm{mmol}$ ) was added over one-half hour to the mixture and the resulting solution was heated to reflux for two hours and then was diluted with $\mathrm{H}_{2} \mathrm{O}(100 \mathrm{~mL})$ and saturated aq $\mathrm{NH}_{4} \mathrm{Cl}(50 \mathrm{~mL})$. The layers were separated and then the organic layer was diluted with concentrated aq $\mathrm{HCl}(100 \mathrm{~mL})$ and heated $\left(80{ }^{\circ} \mathrm{C}\right)$ for five hours. The resulting precipitate was isolated by filtration and added to $0.5 \mathrm{M}$ aq $\mathrm{NaOH}(300 \mathrm{~mL})$. The slurry was then filtered to remove insoluble matter and the resulting solution was acidified with $20 \%$ aq $\mathrm{H}_{2} \mathrm{SO}_{4}$. The resulting precipitate was isolated by filtration to provide phosphinic acid $50(14.5 \mathrm{~g}, 72 \%)$ as a white solid. Spectral data matched those reported in literature: ${ }^{26}{ }^{1} \mathrm{H}$ NMR $\left(400 \mathrm{MHz}, \mathrm{CDCl}_{3}\right) \delta 11.75(\mathrm{~s}, 1 \mathrm{H}), 7.36(\mathrm{~d}, J=12.9,4 \mathrm{H}), 7.07$ (s, 2H), $2.28(\mathrm{~s}, 12 \mathrm{H}) ;{ }^{13} \mathrm{C} \mathrm{NMR}\left(100 \mathrm{MHz}, \mathrm{CDCl}_{3}\right) \delta 138.1(\mathrm{~d}, J=14.0), 133.7,132.8(\mathrm{~d}, J$ $=138.2), 129.0(\mathrm{~d}, J=11.0), 21.4 ;{ }^{31} \mathrm{P}$ NMR $\left(162 \mathrm{MHz}, \mathrm{CDCl}_{3}\right) \delta 32$. HRMS $(\mathrm{ESI}) \mathrm{m} / \mathrm{z}$ calcd for $\mathrm{C}_{16} \mathrm{H}_{19} \mathrm{O}_{2} \mathrm{PH}[\mathrm{M}+\mathrm{H}]^{+} 275.1201$, found 275.1198 .

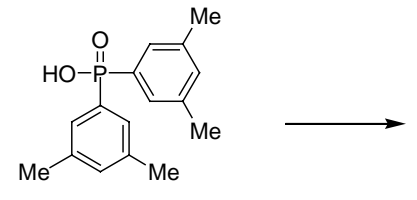

50

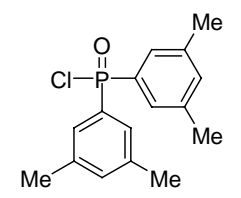

51 
Bis(3,5-dimethylphenyl)phosphonyl chloride (51). ${ }^{26}$ To a heated $\left(50{ }^{\circ} \mathrm{C}\right)$ slurry of phosphinic acid $50(2.5 \mathrm{~g}, 9.2 \mathrm{mmol})$ in toluene $(20 \mathrm{~mL})$ was added thionyl chloride $(1.3$ $\mathrm{mL}, 18 \mathrm{mmol}$ ) over two hours. The reaction mixture was filtered and then was diluted with hexanes $(50 \mathrm{~mL})$. The resulting precipitate was filtered to yield phosphonyl chloride $51(2.7 \mathrm{~g}, 99 \%)$ as a white solid. Spectral data matched those reported in literature: ${ }^{27}{ }^{1} \mathrm{H}$ NMR (400 MHz, $\left.\mathrm{CDCl}_{3}\right) \delta 7.39(\mathrm{~m}, 4 \mathrm{H}), 7.09(\mathrm{~s}, 2 \mathrm{H}), 2.27(\mathrm{~s}, 12 \mathrm{H}) ;{ }^{13} \mathrm{C}$ NMR $(100$ $\left.\mathrm{MHz}, \mathrm{CDCl}_{3}\right) \delta 138.2(\mathrm{~d}, J=7.7), 138.1(\mathrm{~d}, J=7.7), 134.3,130.9(\mathrm{~d}, J=147.4), 130.8$ $(\mathrm{d}, J=147.8), 129.44$ (d, $J=5.9), 129.36(\mathrm{~d}, J=5.9), 21.4 ;{ }^{31} \mathrm{P}$ NMR $\left(162 \mathrm{MHz}, \mathrm{CDCl}_{3}\right)$ $\delta 27$.

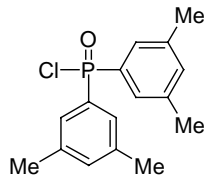

51

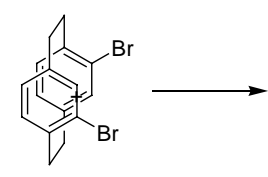

$( \pm)-52$

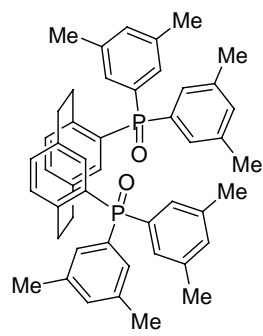

$( \pm)-53$

(士)-pseudo-o-Bis(di(3,5-dimethylphenyl)phosphinyl)[2.2]paracyclophane (53). ${ }^{28}$ To a cooled $\left(-78{ }^{\circ} \mathrm{C}\right)$ solution of dibromide $52(1.7 \mathrm{~g}, 4.5 \mathrm{mmol})$ in THF $(45 \mathrm{~mL})$ was added $t$ BuLi (12 mL, $18 \mathrm{mmol}, 1.5 \mathrm{M}$ solution in pentane) over one-half hour. Magnesium bromide diethyl etherate $(2.7 \mathrm{~g}, 10 \mathrm{mmol})$ was added and the reaction mixture was allowed to warm $\left(10{ }^{\circ} \mathrm{C}\right)$ before adding phosphonyl chloride 51. The resulting reaction mixture was stirred at ambient temperature for 18 hours and then diluted with $\mathrm{Et}_{2} \mathrm{O}(150$ $\mathrm{mL}$ ). The resulting precipitate was collected by filtration. Recrystallization (EtOH) provided bis(phosphine oxide) $53(2.4 \mathrm{~g}, 74 \%)$ as a white crystalline solid. Previous literature report does not provide NMR spectral data: ${ }^{29} \mathrm{H}$ NMR $\left(500 \mathrm{MHz}, \mathrm{CDCl}_{3}\right) \delta$ $7.35(\mathrm{~d}, J=11.8,4 \mathrm{H}), 7.11-7.20(\mathrm{~m}, 6 \mathrm{H}), 7.10(\mathrm{~s}, 2 \mathrm{H}), 7.01(\mathrm{~s}, 2 \mathrm{H}), 6.75(\mathrm{~d}, J=7.1$, 2H), 6.63-6.65 (m, 2H), 3.24-3.27 (m, 2H), 3.13-3.15 (m, 2H), 2.96-3.00 (m, 2H), 2.70$2.73(\mathrm{~m}, 2 \mathrm{H}), 2.40(\mathrm{~s}, 12 \mathrm{H}), 2.23(\mathrm{~s}, 12 \mathrm{H}) ;{ }^{13} \mathrm{C} \mathrm{NMR}\left(125 \mathrm{MHz}, \mathrm{CDCl}_{3}\right) \delta 145.6(\mathrm{~d}, J=$ 8.3), 139.7 (d, $J=13.0), 138.8(\mathrm{~d}, J=13.0), 137.5$ (d, $J=12.5), 137.3$ (d, $J=12.5)$, $136.9(\mathrm{~d}, J=101.3), 136.3(\mathrm{~d}, J=2.9), 134.5(\mathrm{~d}, J=11.8), 133.5(\mathrm{~d}, J=2.8), 132.6(\mathrm{~d}, J$ $=2.6), 132.3(\mathrm{~d}, J=103.4), 131.5(\mathrm{~d}, J=104.2), 129.7$ (d, $J=9.1), 128.5$ (d, $J=9.3)$, $36.2(\mathrm{~d}, J=5.1), 34.8,21.8,21.5 ;{ }^{31} \mathrm{P}$ NMR $\left(162 \mathrm{MHz}, \mathrm{CDCl}_{3}\right) \delta 21$; IR (thin film) 3233, 2860, 1600, 1454, 1272, $1184 \mathrm{~cm}^{-1}$; HRMS (ESI) $\mathrm{m} / \mathrm{z}$ calcd for $\mathrm{C}_{48} \mathrm{H}_{51} \mathrm{O}_{2} \mathrm{P}_{2}[\mathrm{M}+\mathrm{H}]^{+}$ 721.3364 , found 721.3369 .

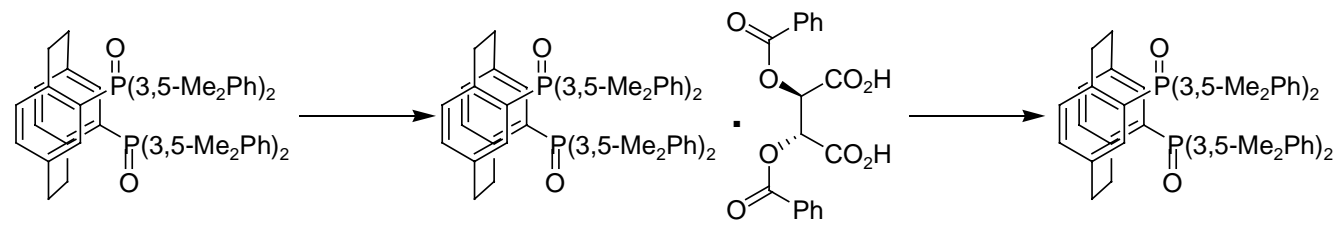

$( \pm)-53$

(R)-53

$(R, R)$-dibenzoyltartaric acid

$(R)-53$

Resolution of (R)-pseudo-o-bis(di(3,5-dimethylphenyl)phosphinyl)[2.2]paracyclophane (53). ${ }^{30}$ To a slurry of bis(phosphine oxide) $( \pm)-53(9.4 \mathrm{~g}, 13 \mathrm{mmol})$ in acetonitrile 
(925 $\mathrm{mL})$ was added (+)-D-dibenzoyltartaric acid $(2.1 \mathrm{~g}, 5.9 \mathrm{mmol})$. The reaction mixture was heated to reflux and allowed to cool slowly (five hours). The resulting precipitate was isolated by filtration and then was dissolved in chloroform $(150 \mathrm{~mL})$. This solution was washed with $1 \mathrm{~N}$ aq NaOH $(2 \times 75 \mathrm{~mL})$ and then with brine $(100 \mathrm{~mL})$. The organic layer was dried over $\mathrm{MgSO}_{4}$ and concentrated in vacuo to yield bis(phosphine oxide) $(R)-53(2.0 \mathrm{~g}, 43 \%)$ as a white solid: $[\alpha]_{\mathrm{D}}=-112^{\circ}\left(\mathrm{c} 1.30, \mathrm{CHCl}_{3}\right)$. Repeating this procedure with (-)-L-dibenzoyltartaric acid provides access to (S)-53. (Note: Oxidation of a commercial sample of $(R)-10$, labeled as " $>95 \%$ ee", to bis(phosphine oxide) $(R)-53$ gave a rotation of $[\alpha]_{\mathrm{D}}=-101.5^{\circ}\left(\right.$ c 2.4, $\left.\mathrm{CHCl}_{3}\right)$.)

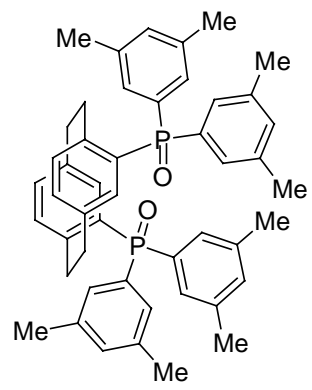

$(R)-53$

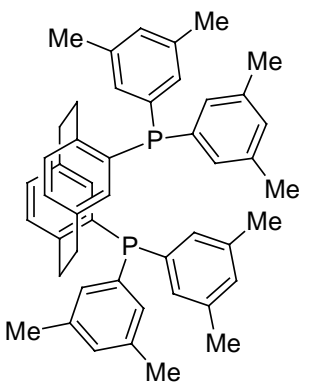

$(R)-10$

(R)-pseudo-o-Bis(di(3,5-dimethylphenyl)phosphino)[2.2]paracyclophane (10). ${ }^{28}$ To a solution of bis(phosphine oxide) $(R)-53(2.2 \mathrm{~g}, 3.1 \mathrm{mmol})$ in $m$-xylene $(50 \mathrm{~mL})$ was added $\mathrm{Cl}_{3} \mathrm{SiH}(5.9 \mathrm{~mL}, 59 \mathrm{mmol})$ and the reaction vessel was sealed. The reaction mixture was heated $\left(140{ }^{\circ} \mathrm{C}\right)$ for 18 hours and then was partitioned between EtOAc $(250$ $\mathrm{mL})$ and $\mathrm{H}_{2} \mathrm{O}(200 \mathrm{~mL})$. The organic layer was washed with brine $(100 \mathrm{~mL})$, dried over $\mathrm{MgSO}_{4}$, and concentrated in vacuo. Purification by flash chromatography (hexanes/EtOAc 19:1) yielded bisphosphine 10 (1.9 g, 90\%) as a white solid. Previous literature report does not provide NMR spectral data: ${ }^{29}[\alpha]_{\mathrm{D}}=-44^{\circ}\left(\mathrm{c} 1.2, \mathrm{CHCl}_{3}\right) ;{ }^{1} \mathrm{H}$ $\operatorname{NMR}\left(500 \mathrm{MHz}, \mathrm{CDCl}_{3}\right) \delta 7.23(\mathrm{~d}, J=8.5,4 \mathrm{H}), 7.10(\mathrm{~d}, J=8.2,4 \mathrm{H}), 7.02(\mathrm{~s}, 2 \mathrm{H}), 6.90$ $(\mathrm{s}, 2 \mathrm{H}), 6.60(\mathrm{~d}, J=9.2,2 \mathrm{H}), 6.53-6.57(\mathrm{~m}, 4 \mathrm{H}), 2.97-3.02(\mathrm{~m}, 2 \mathrm{H}), 2.86-2.92(\mathrm{~m}, 4 \mathrm{H})$, 2.59-2.62 (m, 2H), $2.37(\mathrm{~s}, 12 \mathrm{H}), 2.23(\mathrm{~s}, 12 \mathrm{H}) ;{ }^{13} \mathrm{C} \mathrm{NMR}\left(125 \mathrm{MHz}, \mathrm{CDCl}_{3}\right) \delta 143.4(\mathrm{~d}$, $J=13.8), 139.3(\mathrm{~d}, J=11.3), 139.1(\mathrm{~d}, J=2.5), 138.4(\mathrm{~d}, J=11.3), 137.9$ (d, $J=11.3)$, $137.7(\mathrm{~d}, J=8.8), 137.6(\mathrm{~d}, J=8.8), 134.2(\mathrm{~d}, J=2.5), 133.5(\mathrm{~d}, J=3.8), 133.4(\mathrm{~d}, J=$ 21.3), 133.3 (d, $J=3.8), 132.5,131.1(\mathrm{~d}, J=21.3), 131.0(\mathrm{~d}, J=94), 35.9$ (d, $J=6.3)$, 33.4, 21.7, $21.5^{31} \mathrm{P}$ NMR (162 MHz, $\mathrm{CDCl}_{3}$ ) $\delta$-2; IR (thin film) 3258, 2918, 2855, 1452, 1123, $1038 \mathrm{~cm}^{-1}$; HRMS (ESI) $\mathrm{m} / \mathrm{z}$ calcd for $\mathrm{C}_{48} \mathrm{H}_{50} \mathrm{O}_{2} \mathrm{P}_{2} \mathrm{H}[\mathrm{M}+\mathrm{H}]^{+} 721.3364$ found 721.3369. Anal. Calcd for $\mathrm{C}_{48} \mathrm{H}_{50} \mathrm{P}_{2}$ : C, 83.69; H, 7.32. Found: C, 83.58; H, 7.43.

\section{Kinetic Resolutions}

All reactions were run in triplicate and the experiments shown here are typical. Values for $k_{\text {rel }}$ were calculated using the equation reported by Kagan: $k_{\text {rel }}=\ln$ $\left[\left((1-\mathrm{C})\left(1-\mathrm{ee}_{\mathrm{sm}}\right)\right) /\left((1-\mathrm{C})\left(1+\mathrm{ee}_{\mathrm{sm}}\right)\right)\right]$, in which $\mathrm{C}$ is the fractional conversion of the starting material and $e_{\mathrm{sm}}$ is the enantiomeric excess of the recovered starting material. ${ }^{31}$ 
(Note: In most cases, conversions were determined by HPLC analysis due to overlapping peaks in the ${ }^{1} \mathrm{H}$ NMR spectra of the alcohols and hydroperoxides. The values obtained, however, are all within 3\% of conversions determined by single pulse ${ }^{1} \mathrm{H}$ NMR analysis. The error-bars associated with the $k_{\text {rel }}$ 's obtained from this type of analysis are unsymmetrical and large due to the logarithmic nature of the equations used (vide supra). For example: a resolution with a measured ee of $63 \%$ and conversion of $43 \%$ correlates to a $k_{\text {rel }}$ of 21 . When the same equation is utilized to determine the $k_{\text {rel }}$ from the error limits, $40 \%$ and $46 \%$, the values are 13 and 68, respectively. We feel the values reported in this communication are representative of the values that would be obtained from calculating the $k_{\text {rel }}$ 's from re-isolated starting material.)

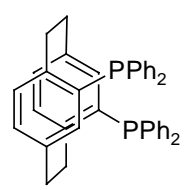

$(R)-9$

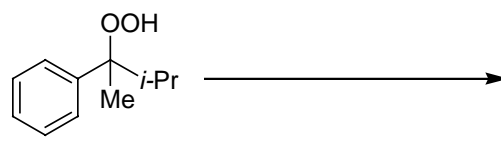

$( \pm)-11$

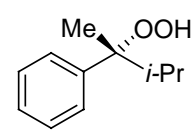

$(+)-(R)-11$

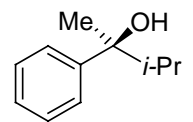

$(-)-(S)-12$

Representative procedure for kinetic resolution; Table 1, Entry 8: To a cooled (-67 ${ }^{\circ} \mathrm{C}$ ) solution of 3-methyl-2-phenylbut-2-yl hydroperoxide (11) (49 $\mathrm{mg}, 0.27 \mathrm{mmol}$ ) in $\mathrm{CDCl}_{3}(0.7 \mathrm{~mL})$ was added a solution of $(R)-(+)-4,12$-bis(diphenylphosphino)-[2.2]paracyclophane (9) $(39 \mathrm{mg}, 0.070 \mathrm{mmol})$ in $\mathrm{CDCl}_{3}(0.2 \mathrm{~mL})$ by cannula. The phosphine residue-containing vessel was washed with $\mathrm{CDCl}_{3}(0.3 \mathrm{~mL})$ and the resulting solution was transferred by cannula. After 16 hours, the reaction mixture was allowed to warm $\left(25{ }^{\circ} \mathrm{C}\right)$ and the fractional conversion was measured by ${ }^{1} \mathrm{H}$ NMR spectroscopy $(0.55)$. The reaction mixture was concentrated and filtered through a plug of silica $(100 \%$ $\mathrm{CH}_{2} \mathrm{Cl}_{2}$ ) yielding a mixture of 3-methyl-2-phenylbut-2-yl hydroperoxide (11) and 3methyl-2-phenylbutan-2-ol (12) (45 mg). The ee of recovered starting material $(75 \%)$ was determined by HPLC [Chiracel OD-H column $(250 \mathrm{~mm} \times 4.6 \mathrm{~mm}$ i.d. $)$ with $i-$ $\mathrm{PrOH} /$ hexanes $(5: 95 \mathrm{v} / \mathrm{v})$, flow rate $0.8 \mathrm{~mL} \cdot \mathrm{min}^{-1}$, detection by $\mathrm{UV}$ absorbance at 254 $\mathrm{nm}$, retention times: $(+)-(R)-\mathbf{1 1}=15.1 \mathrm{~min}$ (major) and $(-)-(S)-\mathbf{1 1}=22.2 \mathrm{~min}$ (minor)]. An ee of $75 \%$ with a conversion of 0.55 corresponds to a $k_{\text {rel }}$ of 4.2 .

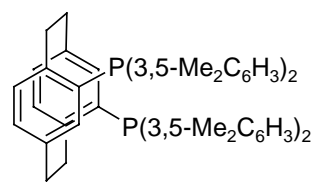

$(R)-10$

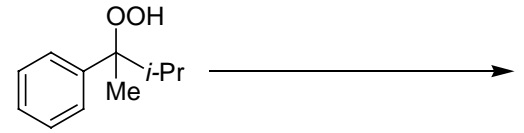

$( \pm)-11$

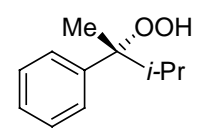

$(+)-(R)-11$

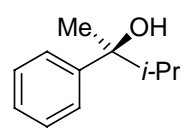

$(-)-(S)-12$

Table 1, Entry 9: The representative procedure was followed using compound 11 (11 $\mathrm{mg}, 0.060 \mathrm{mmol})$ and compound $(R)-10(18 \mathrm{mg}, 0.025 \mathrm{mmol})$. The reaction mixture was filtered through a plug of silica $\left(100 \% \mathrm{CH}_{2} \mathrm{Cl}_{2}\right)$ yielding a mixture of hydroperoxide 11 and alcohol $12(9 \mathrm{mg})$. The conversion (0.50) and ee of recovered starting material $(86 \%)$ were determined by HPLC [Chiracel OD-H column $(250 \mathrm{~mm} \times 4.6 \mathrm{~mm}$ i.d.) with $i$-PrOH/hexanes $(5: 95 \mathrm{v} / \mathrm{v})$, flow rate $0.8 \mathrm{~mL} \cdot \mathrm{min}^{-1}$, detection by UV absorbance at 254 $\mathrm{nm}$, retention times: $(+)-(R)-\mathbf{1 1}=15.1 \mathrm{~min}$ (major) and $(-)-(S)-\mathbf{1 1}=22.2 \mathrm{~min}$ (minor) and correspond to a $k_{\text {rel }}$ of 36 . 


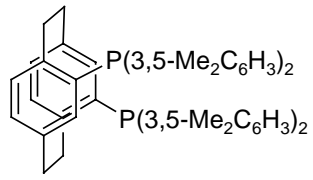

(R)-10

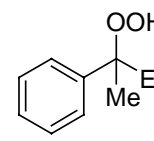

$( \pm)-13$

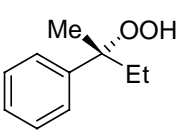

$(+)-(R)-13$

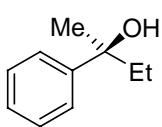

$(-)-(S)-42$

Table 2, Entry 1: The representative procedure was followed using compound 13 (9.0 $\mathrm{mg}, 0.054 \mathrm{mmol})$ and compound $(R)-10(9.3 \mathrm{mg}, 0.014 \mathrm{mmol})$. The reaction mixture was filtered through a plug of silica $\left(100 \% \mathrm{CH}_{2} \mathrm{Cl}_{2}\right)$ yielding a mixture of hydroperoxide 13 and alcohol $42(8 \mathrm{mg})$. The conversion $(0.43)$ and ee of recovered starting material $(63 \%)$ were determined by HPLC [Chiracel OD-H column $(250 \mathrm{~mm} \times 4.6 \mathrm{~mm}$ i.d.) with $i$-PrOH/hexanes $(5: 95 \mathrm{v} / \mathrm{v})$, flow rate $0.8 \mathrm{~mL} \cdot \mathrm{min}^{-1}$, detection by UV absorbance at 210 $\mathrm{nm}$, retention times: $(+)-(R)-\mathbf{1 3}=15.3 \mathrm{~min}$ (major) and $(-)-(S)-\mathbf{1 3}=21.1 \mathrm{~min}$ (minor) and correspond to a $k_{\text {rel }}$ of 21 .

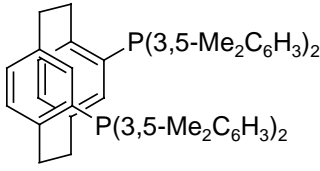

(S)-10

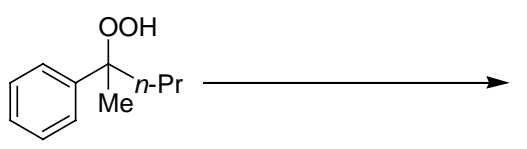

$( \pm)-14$

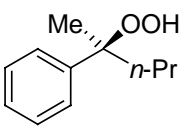

$(-)-(S)-14$

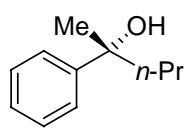

$(+)-(R)-28$

Table 2, Entry 2: The representative procedure was followed using compound 14 (32 $\mathrm{mg}, 0.18 \mathrm{mmol})$ and compound $(S)-10(30 \mathrm{mg}, 0.044 \mathrm{mmol})$. The reaction mixture was filtered through a plug of silica $\left(100 \% \mathrm{CH}_{2} \mathrm{Cl}_{2}\right)$ yielding a mixture of hydroperoxide 14 and alcohol 28 (22 mg). The conversion (0.46) was determined by ${ }^{1} \mathrm{H}$ NMR and ee of recovered starting material $(71 \%)$ was determined by HPLC [Chiracel OD-H column $\left(250 \mathrm{~mm} \times 4.6 \mathrm{~mm}\right.$ i.d.) with $i-\mathrm{PrOH} /$ hexanes $(5: 95 \mathrm{v} / \mathrm{v})$, flow rate $0.8 \mathrm{~mL} \cdot \mathrm{min}^{-1}$, detection by UV absorbance at $254 \mathrm{~nm}$, retention times: $(+)-(R)-\mathbf{1 4}=12.2 \mathrm{~min}$ (minor) and $(-)-(S)-14=16.4 \min \left(\right.$ major)] and correspond to a $k_{\text {rel }}$ of 23.

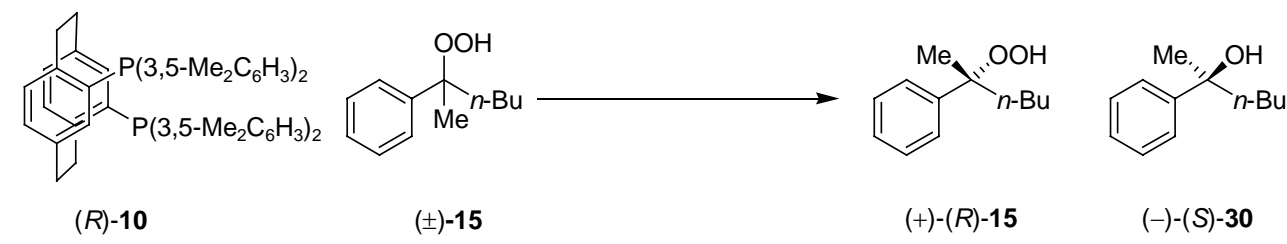

Table 2, Entry 3: The representative procedure was followed using compound 15 (14 $\mathrm{mg}, 0.073 \mathrm{mmol})$ and compound $(R)-10(13 \mathrm{mg}, 0.018 \mathrm{mmol})$. The reaction mixture was filtered through a plug of silica $\left(100 \% \mathrm{CH}_{2} \mathrm{Cl}_{2}\right)$ yielding a mixture of hydroperoxide 15 and alcohol $30(13 \mathrm{mg})$. The conversion (0.57) and ee of recovered starting material $(90 \%)$ were determined by HPLC [Chiracel OD-H column $(250 \mathrm{~mm} \times 4.6 \mathrm{~mm}$ i.d.) with $i$-PrOH/hexanes $(5: 95 \mathrm{v} / \mathrm{v})$, flow rate $0.8 \mathrm{~mL} \cdot \mathrm{min}^{-1}$, detection by $\mathrm{UV}$ absorbance at 254 $\mathrm{nm}$, retention times: $(+)-(R)-\mathbf{1 5}=14.1 \mathrm{~min}$ (major) and $(-)-(S)-\mathbf{1 5}=18.9 \mathrm{~min}$ (minor) and correspond to a $k_{\text {rel }}$ of 16 . 


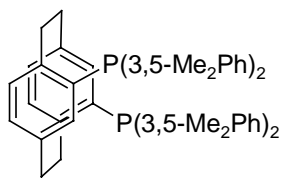

$(R)-10$

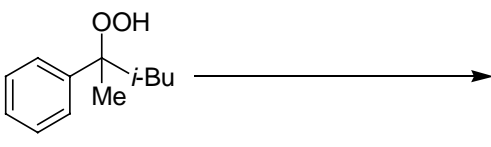

$( \pm)-16$<smiles>O=C(O)O[C@]([Mg])(Br)c1ccccc1</smiles>

$(R)-16$<smiles>OC(O)([181I])c1ccccc1</smiles>

(S) -32

Table 2, Entry 4: The representative procedure was followed using compound 16 (27 $\mathrm{mg}, 0.14 \mathrm{mmol})$ and compound $(R)-\mathbf{1 0}(24 \mathrm{mg}, 0.035 \mathrm{mmol})$. The reaction mixture was filtered through a plug of silica $\left(100 \% \mathrm{CH}_{2} \mathrm{Cl}_{2}\right)$ yielding a mixture of hydroperoxide $\mathbf{1 6}$ and alcohol $32(25 \mathrm{mg})$. The conversion (0.39) and ee of recovered starting material (58\%) were determined by HPLC [Chiracel OD-H column $(250 \mathrm{~mm} \times 4.6 \mathrm{~mm}$ i.d.) with $i$-PrOH/hexanes $(5: 95 \mathrm{v} / \mathrm{v})$, flow rate $0.8 \mathrm{~mL} \cdot \mathrm{min}^{-1}$, detection by $\mathrm{UV}$ absorbance at 254 $\mathrm{nm}$, retention times: $(+)-(R)-\mathbf{1 6}=11.3 \mathrm{~min}$ (major) and $(-)-(S)-\mathbf{1 6}=17.1 \mathrm{~min}$ (minor)] and correspond to a $k_{\text {rel }}$ of 37 .

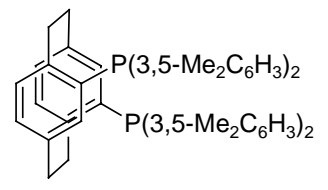

$(R)-10$

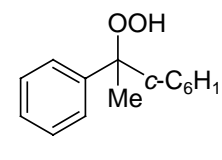

$( \pm)-17$

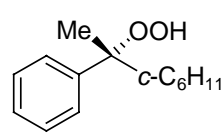

$(+)-(R)-17$

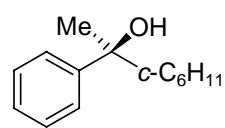

$(-)-(S)-34$

Table 2, Entry 5: The representative procedure was followed using compound 17 (96 $\mathrm{mg}, 0.43 \mathrm{mmol})$ and compound $(R)-10(100 \mathrm{mg}, 0.145 \mathrm{mmol})$. The reaction mixture was filtered through a plug of silica $\left(100 \% \mathrm{CH}_{2} \mathrm{Cl}_{2}\right)$ yielding a mixture of hydroperoxide 17 and alcohol $34(25 \mathrm{mg})$. The conversion (0.56) and ee of recovered starting material $(95 \%)$ were determined by HPLC [Chiracel OD-H column $(250 \mathrm{~mm} \times 4.6 \mathrm{~mm}$ i.d.) with $i$-PrOH/hexanes $(5: 95 \mathrm{v} / \mathrm{v})$, flow rate $0.8 \mathrm{~mL} \cdot \mathrm{min}^{-1}$, detection by $\mathrm{UV}$ absorbance at 254 $\mathrm{nm}$, retention times: $(+)-(R)-\mathbf{1 7}=16.2 \mathrm{~min}$ (major) and $(-)-(S)-\mathbf{1 7}=32.0 \mathrm{~min}$ (minor)] and correspond to a $k_{\text {rel }}$ of 25. A sample of hydroperoxide $(R)-(+)-\mathbf{1 7}$ was purified by semi-prep HPLC [Alltech Altima column $(250 \mathrm{~mm} \times 22 \mathrm{~mm}$ i.d. $)$ with EtOAc/hexanes $(3: 97 \mathrm{v} / \mathrm{v})$, flow rate $\left.10 \mathrm{~mL} \cdot \mathrm{min}^{-1}\right):[\alpha]_{\mathrm{D}}=+64^{\circ}\left(c=2.3, \mathrm{CHCl}_{3}\right)$. NMR spectral data matched previously reported data (vide supra).

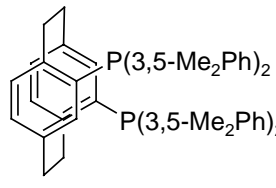

$(R)-10$

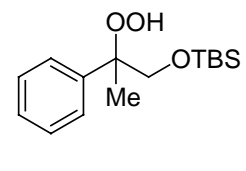

$( \pm)-18$

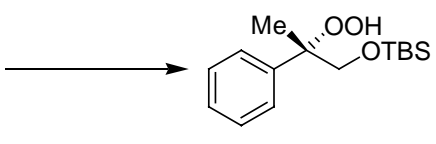

$(+)$ - or $(-)-18$

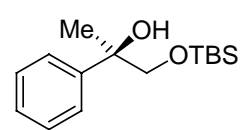

$(+)$ - or $(-)-54$

Table 2, Entry 6: The representative procedure was followed using compound 18 (30 $\mathrm{mg}, 0.11 \mathrm{mmol})$ and compound $(R)-10(18 \mathrm{mg}, 0.027 \mathrm{mmol})$. The reaction mixture was filtered through a plug of silica $\left(100 \% \mathrm{CH}_{2} \mathrm{Cl}_{2}\right)$ yielding a mixture of hydroperoxide 18 and alcohol $54(25 \mathrm{mg})$. The conversion (0.49) was determined by ${ }^{1} \mathrm{H}$ NMR. The ee of recovered starting material $(42 \%)$ was determined by HPLC [Chiracel OD-H column $\left(250 \mathrm{~mm} \times 4.6 \mathrm{~mm}\right.$ i.d.) with $i-\mathrm{PrOH} /$ hexanes $(5: 95 \mathrm{v} / \mathrm{v})$, flow rate $0.8 \mathrm{~mL} \cdot \mathrm{min}^{-1}$, detection by UV absorbance at $254 \mathrm{~nm}$, retention times: (major)-18 $=9.5 \mathrm{~min}$ and (minor)-18 $=11.7 \mathrm{~min}]$ and the two correspond to a $k_{\text {rel }}$ of 3.8 . 


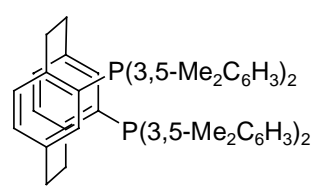

(R)-10

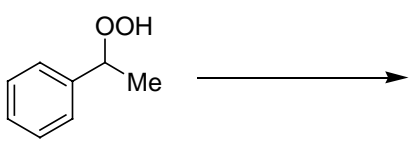

$( \pm)-19$

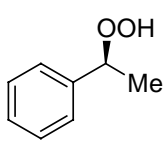

$(+)-(R)-19$

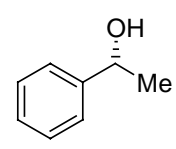

$(-)-(S)-46$

Table 2, Entry 7: The representative procedure was followed using compound 19 (10 $\mathrm{mg}, 0.075 \mathrm{mmol})$ and compound $(R)-\mathbf{1 0}(17 \mathrm{mg}, 0.025 \mathrm{mmol})$. The reaction mixture was filtered through a plug of silica $\left(100 \% \mathrm{CH}_{2} \mathrm{Cl}_{2}\right)$ yielding a mixture of hydroperoxide $\mathbf{1 9}$ and alcohol $46(7 \mathrm{mg})$. The conversion (0.84) and ee of recovered starting material (77\%) were determined by HPLC [Chiracel OD-H column $(250 \mathrm{~mm} \times 4.6 \mathrm{~mm}$ i.d. $)$ with i-PrOH/hexanes $(5: 95 \mathrm{v} / \mathrm{v})$, flow rate $0.8 \mathrm{~mL} \cdot \mathrm{min}^{-1}$, detection by UV absorbance at 210 nm, retention times: $(+)-(R)-\mathbf{1 9}=16.6$ min (major) and $(-)-(S)-\mathbf{1 9}=24.7$ min (minor)] and correspond to a $k_{\text {rel }}$ of 2.6 .

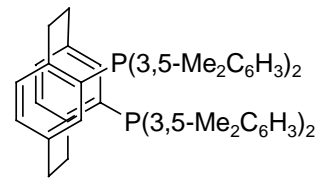

(R)-10

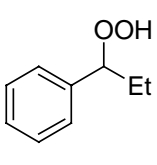

$( \pm)-20$

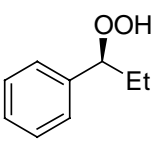

$(+)-(R)-20$

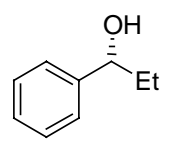

(-)-(S)-47

Table 2, Entry 8: The representative procedure was followed using compound 20 (9.0 $\mathrm{mg}, 0.057 \mathrm{mmol})$ and compound $(R)-\mathbf{1 0}(10 \mathrm{mg}, 0.014 \mathrm{mmol})$. The reaction mixture was filtered through a plug of silica $\left(100 \% \mathrm{CH}_{2} \mathrm{Cl}_{2}\right)$ yielding a mixture of hydroperoxide $\mathbf{2 0}$ and alcohol $47(7 \mathrm{mg})$. The conversion (0.75) and ee of recovered starting material (76\%) were determined by HPLC [Chiracel OD-H column $(250 \mathrm{~mm} \times 4.6 \mathrm{~mm}$ i.d.) with $i$-PrOH/hexanes $(5: 95 \mathrm{v} / \mathrm{v})$, flow rate $0.8 \mathrm{~mL} \cdot \mathrm{min}^{-1}$, detection by UV absorbance at 210 $\mathrm{nm}$, retention times: $(+)-(R)-\mathbf{2 0}=18.7$ min (major) and $(-)-(S)-\mathbf{2 0}=23.4 \mathrm{~min}($ minor) $]$ and correspond to a $k_{\text {rel }}$ of 3.4 .

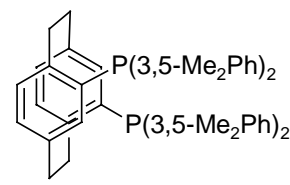

(R)-10

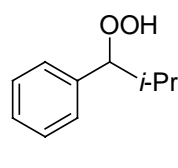

$( \pm)-21$

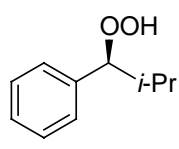

(+)-(R)-21

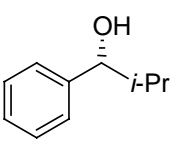

(-)-(S)-38

Table 2, Entry 9: The representative procedure was followed using compound 21 (18 $\mathrm{mg}, 0.11 \mathrm{mmol})$ and compound $(R)-\mathbf{1 0}(24 \mathrm{mg}, 0.035 \mathrm{mmol})$. The reaction mixture was filtered through a plug of silica $\left(100 \% \mathrm{CH}_{2} \mathrm{Cl}_{2}\right)$ yielding a mixture of hydroperoxide 21 and alcohol $38(14 \mathrm{mg})$. The conversion (0.47) and ee of recovered starting material (54\%) were determined by HPLC [Chiracel OD-H column $(250 \mathrm{~mm} \times 4.6 \mathrm{~mm}$ i.d. $)$ with i-PrOH/hexanes $(5: 95 \mathrm{v} / \mathrm{v})$, flow rate $0.8 \mathrm{~mL} \cdot \mathrm{min}^{-1}$, detection by UV absorbance at 254 nm, retention times: $(+)-(R)-21=14.5$ min (major) and $(-)-(S)-21=24.3$ min (minor)] and correspond to a $k_{\text {rel }}$ of 6.9. 


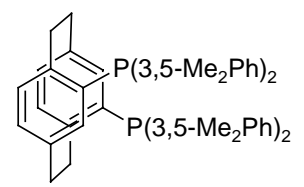

(R)-10

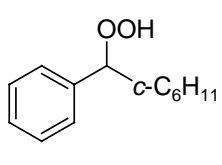

(士)-22

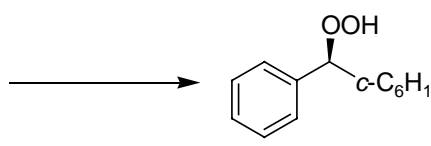

(R)-22

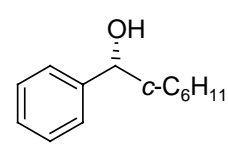

(S)-41

Table 2, Entry 10: The representative procedure was followed using compound 22 (21 $\mathrm{mg}, 0.10 \mathrm{mmol})$ and compound $(R)-\mathbf{1 0}(20 \mathrm{mg}, 0.029 \mathrm{mmol})$. The reaction mixture was filtered through a plug of silica $\left(100 \% \mathrm{CH}_{2} \mathrm{Cl}_{2}\right)$ yielding a mixture of hydroperoxide 22 and alcohol $41(19 \mathrm{mg})$. The conversion (0.43) and ee of recovered starting material $(42 \%)$ were determined by HPLC [Chiracel OD-H column $(250 \mathrm{~mm} \times 4.6 \mathrm{~mm}$ i.d. $)$ with i-PrOH/hexanes $(5: 95 \mathrm{v} / \mathrm{v})$, flow rate $0.8 \mathrm{~mL} \cdot \mathrm{min}^{-1}$, detection by UV absorbance at 210 nm, retention times: $(+)-(R)-22=14.9$ min (major) and $(-)-(S)-22=22.9$ min (minor)] and correspond to a $k_{\text {rel }}$ of 5.2.

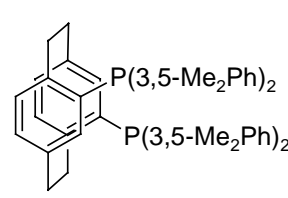

(R)-10

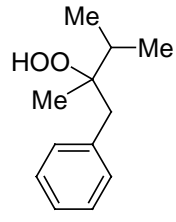

(土)-23

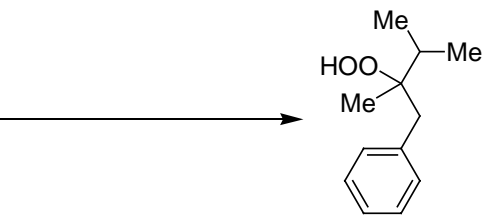

(+) or (-)-23

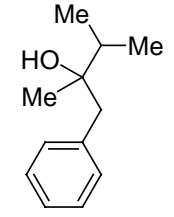

(+) or (-)-39

Scheme 1, entry 1: The representative procedure was followed using compound 23 (23 $\mathrm{mg}, 0.11 \mathrm{mmol})$ and compound $(R)-\mathbf{1 0}(20 \mathrm{mg}, 0.028 \mathrm{mmol})$. The reaction mixture was concentrated and conversion was measured with ${ }^{1} \mathrm{H}$ NMR (.45). Purification by chromatography (hexanes/EtOAc 19:1) yielded hydroperoxide $23(9 \mathrm{mg})$ as a clear oil. The ee of recovered starting material (51\%) was determined by HPLC [Chiracel OB-H column $(2 \times 250 \mathrm{~mm} \times 4.6 \mathrm{~mm}$ i.d. $)$ with $i-\mathrm{PrOH} /$ hexanes $(5: 95 \mathrm{v} / \mathrm{v})$, flow rate 0.8 $\mathrm{mL} \cdot \mathrm{min}^{-1}$, detection by UV absorbance at $254 \mathrm{~nm}$, retention times: major $=10.6 \mathrm{~min}$ and minor $=11.9 \mathrm{~min}]$ and correspond to a $k_{\mathrm{rel}}$ of 7.1.

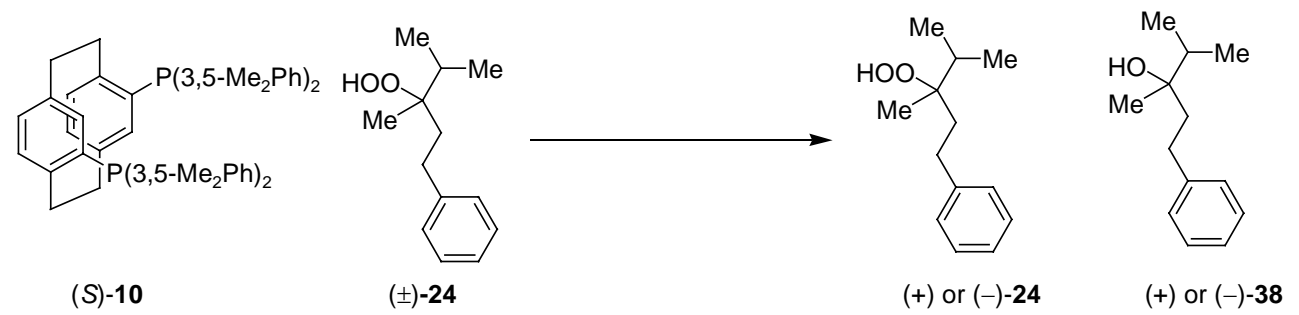

Scheme 1, entry 2: The representative procedure was followed using compound 24 (38 $\mathrm{mg}, 0.13 \mathrm{mmol})$ and compound $(S)-\mathbf{1 0}(42 \mathrm{mg}, 0.030 \mathrm{mmol})$. The reaction mixture was purified by chromatography (hexanes/EtOAc 19:1) yielding hydroperoxide 24 (13 mg). The conversion (0.64) and ee of recovered starting material $(0 \%)$ were determined by HPLC [Chiracel OB-H column $(2 \times 250 \mathrm{~mm} \times 4.6 \mathrm{~mm}$ i.d. $)$ with $i$-PrOH/hexanes $(5: 95$ $\mathrm{v} / \mathrm{v}$ ), flow rate $0.8 \mathrm{~mL} \cdot \mathrm{min}^{-1}$, detection by UV absorbance at $254 \mathrm{~nm}$, retention times: minor $=20.4 \mathrm{~min}$ and major $=22.3 \mathrm{~min}]$ and correspond to a $k_{\mathrm{rel}}$ of 1.0 . 


\section{Isolation of Enantiopure Hydroperoxide}

The information in this section describes efforts to utilize the resolution conditions to isolate appreciable amounts of enantiopure hydroperoxides (Scheme 4). The resolution of hydroperoxide $( \pm)$-11 with $(R)$-xylyl-PHANEPHOS (10) was taken to $\sim 70 \%$ conversion to ensure recovered starting material with $>99 \%$ ee. In the course of developing this methodology, it became apparent that isolation of the recovered starting material by flash chromatography was not a viable option for all substrates; however, selective protection of the hydroperoxide in the presence of the alcohol was possible. After isolation of the silylperoxyether 55 by chromatography and subsequent deprotection, enantiopure hydroperoxide $(+)-(R)-\mathbf{1 1}$ was obtained. Reduction of hydroperoxide $(+)-(R)-\mathbf{1 1}$ with $\mathrm{PPh}_{3}$ provided enantiopure alcohol $(+)-(R)-\mathbf{1 2}$.

\section{Scheme 2.}

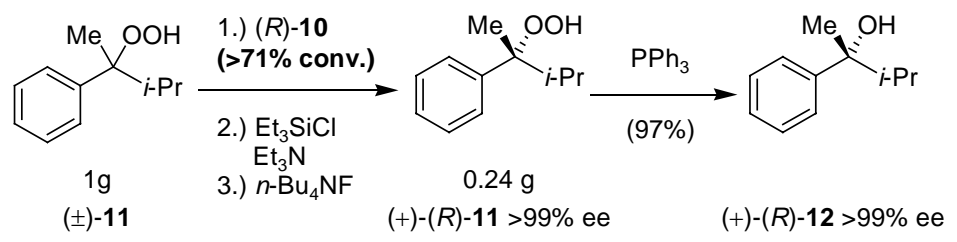

Large-scale resolution of $( \pm)$-3-methyl-2-phenylbut-2-yl hydroperoxide (11). To a cooled $\left(-67{ }^{\circ} \mathrm{C}\right)$ solution of 3-methyl-2-phenylbut-2-yl hydroperoxide (11) (1.0 g, 5.6 mmol $)$ in $\mathrm{CDCl}_{3}(12 \mathrm{~mL})$ was added a cooled $\left(-67{ }^{\circ} \mathrm{C}\right)$ solution of $(R)$-xylyl PHANEPHOS (10) $(1.3 \mathrm{~g}, 1.8 \mathrm{mmol})$ in $\mathrm{CDCl}_{3}(4.0 \mathrm{~mL})$ by cannula. Additional $\mathrm{CDCl}_{3}$ $(4.0 \mathrm{~mL})$ was used to rinse the phosphine residue-containing vessel, and the resulting solution was transferred by cannula to the reaction mixture. The reaction mixture was stirred at $-67{ }^{\circ} \mathrm{C}$ for 16 hours and the fractional conversion was measured by ${ }^{1} \mathrm{H}$ NMR spectroscopy $(0.71)$. The reaction mixture was concentrated and filtered through a plug of silica $\left(100 \% \mathrm{CH}_{2} \mathrm{Cl}_{2}\right)$ yielding a mixture of 3-methyl-2-phenylbut-2-yl hydroperoxide (11) and 3-methyl-2-phenylbutan-2-ol (12) (0.90 g). The ee of recovered starting material $(>99 \%)$ was determined by SFC [Chiracel OD-H column $(250 \mathrm{~mm} \times 4.6 \mathrm{~mm}$ i.d.) with $\mathrm{MeOH}: \mathrm{CO}_{2}(3: 97 \mathrm{v} / \mathrm{v})$, flow rate $2.5 \mathrm{~mL} \cdot \mathrm{min}^{-1}$, detection by $\mathrm{UV}$ absorbance at $210 \mathrm{~nm}$, retention times: $(+)-(R)-\mathbf{1 1}=6.4 \mathrm{~min}$ (major) and $(-)-(S)-\mathbf{1 1}=10.8 \mathrm{~min}$ (minor)] and corresponds to a $k_{\text {rel }}>11$.

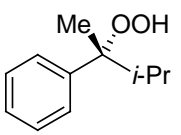

$(+)-(R)-11$

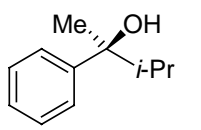

$(-)-(S)-12$

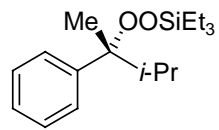

(R)-55

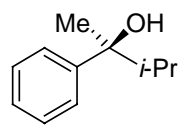

$(-)-(S)-12$

3-Methyl-2-phenylbut-2-ylhydroperoxytrimethylsilyl ether (55). To a cooled $\left(0{ }^{\circ} \mathrm{C}\right)$ solution of hydroperoxide $(+)-(R)-11(0.26 \mathrm{~g}, 1.4 \mathrm{mmol})$ and alcohol $(-)-(S)-12(0.64 \mathrm{~g}$, $3.9 \mathrm{mmol})$ in $\mathrm{CH}_{2} \mathrm{Cl}_{2}(5 \mathrm{~mL})$ were added $\mathrm{Et}_{3} \mathrm{SiCl}(0.35 \mathrm{~mL}, 2.1 \mathrm{mmol})$ and $\mathrm{Et}_{3} \mathrm{~N}(0.58$ $\mathrm{mL}, 4.2 \mathrm{mmol})$. The reaction mixture was stirred for two hours and then diluted with 
EtOAc $(40 \mathrm{~mL})$. The solution was washed with $\mathrm{H}_{2} \mathrm{O}(3 \times 20 \mathrm{~mL})$ and brine $(30 \mathrm{~mL})$, dried over $\mathrm{MgSO}_{4}$, and concentrated in vacuo. The resulting oil was purified by flash chromatography $(100 \%$ hexanes) to yield silylperoxy ether $55(0.40 \mathrm{~g}, 95 \%)$. Previous literature report does not provide NMR spectral data: ${ }^{32} \mathrm{H}$ NMR $\left(500 \mathrm{MHz}, \mathrm{CDCl}_{3}\right) \delta$ 7.39-7.40 (m, 2H), 7.30-7.34 (m, 2H), 7.22-7.26 (m, 1H), 1.97- $2.03(\mathrm{~m}, 1 \mathrm{H}), 1.63(\mathrm{~s}$, $3 \mathrm{H}), 1.00(\mathrm{t}, J=7.8,9 \mathrm{H}), 0.88(\mathrm{~d}, 3 \mathrm{H}, J=6.9), 0.76(\mathrm{~d}, J=6.9,3 \mathrm{H}), 0.68-0.73$ (q, $J=$ 8.0, 6H); ${ }^{13} \mathrm{C}$ NMR $\left(125 \mathrm{MHz}, \mathrm{CDCl}_{3}\right) \delta 144.4,127.6,126.9,126.6,88.2,37.8,19.3$, 17.9, 17.8, 7.0, 4.1. Anal. Calcd for $\mathrm{C}_{17} \mathrm{H}_{30} \mathrm{O}_{2} \mathrm{Si}$ : C, 69.33; H, 10.27. Found: C, 69.34; H, 10.50 .

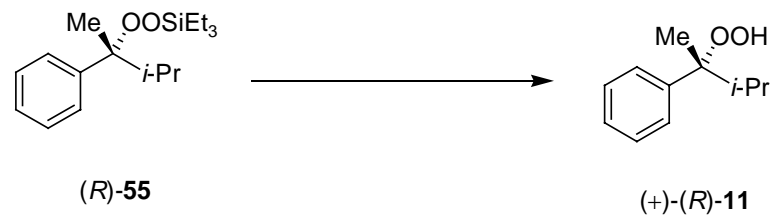

(+)-3-Methyl-2-phenylbut-2-yl hydroperoxide (11). To a cooled $\left(-10{ }^{\circ} \mathrm{C}\right)$ solution of silylperoxy ether $55(0.40 \mathrm{~g}, 1.4 \mathrm{mmol})$ in THF $(10 \mathrm{~mL})$ was added $n$-Bu $\mathrm{H}_{4} \mathrm{NF}(1.5 \mathrm{~mL}, 1.5$ mmol, 1.0 $\mathrm{M}$ in THF). The reaction mixture was diluted with $1 \mathrm{~N}$ aq $\mathrm{HCl}(20 \mathrm{~mL})$ and was then extracted with EtOAc $(3 \times 25 \mathrm{~mL})$. The combined organic layers were washed with brine $(50 \mathrm{~mL})$, dried over $\mathrm{MgSO}_{4}$, and concentrated in vacuo. The resulting oil was purified by flash chromatography (hexanes/EtOAc 19:1) to yield enantiopure hydroperoxide $(+)-(R)-11(0.24 \mathrm{~g}, 99 \%):[\alpha]_{\mathrm{D}}=+87^{\circ}\left(c=3.1, \mathrm{CHCl}_{3}\right)$. NMR spectral data matched previously reported data (vide supra).

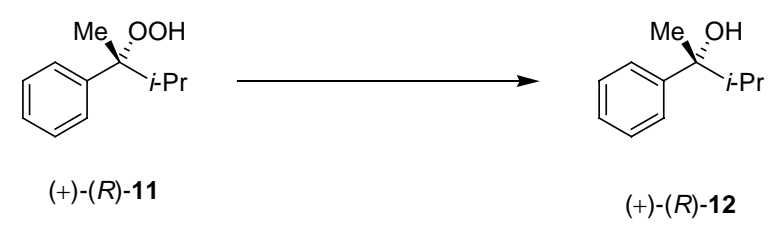

(+)-3-Methyl-2-phenylbutan-2-ol (12). To a cooled $\left(0{ }^{\circ} \mathrm{C}\right)$ solution of hydroperoxide (+)-11 (0.09 g, $0.5 \mathrm{mmol})$ in $\mathrm{CHCl}_{3}(4.0 \mathrm{~mL})$ was added $\mathrm{PPh}_{3}(0.13 \mathrm{~g}, 0.50 \mathrm{mmol})$. The reaction mixture was stirred for one-half hour, concentrated in vacuo and purified by flash chromatography (hexanes/EtOAc 19:1) to yield enantiopure alcohol $(+)-(R)-12$ $(0.070,95 \%):[\alpha]_{\mathrm{D}}=+30^{\circ}\left(c=2.4, \mathrm{CHCl}_{3}\right)$; this data is in contrast to that reported by Walsh et. al., $[\alpha]_{\mathrm{D}}=+6.0^{\circ}\left(c=0.05, \mathrm{CHCl}_{3}\right)$, for material with $75 \%$ ee. ${ }^{33} \mathrm{NMR}$ spectral data matched previously reported data (vide supra).

VI. Resolution with Mono(phosphine oxide) 25. 


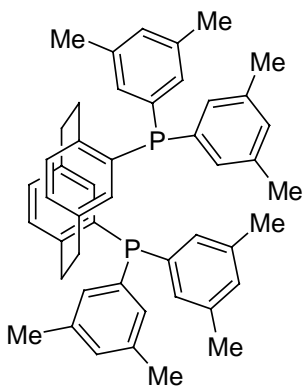

$(R)-10$

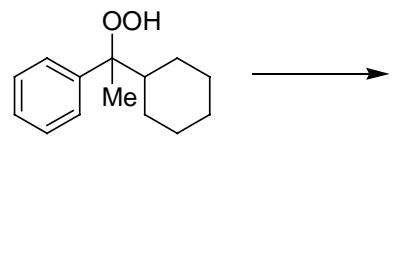

$( \pm)-17$

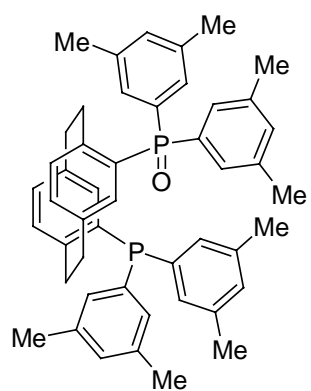

$(R)-25$

\section{(R)-pseudo-o-(Di(3,5-dimethylphenyl)phosphino)-(di(3,5-dimethylphen-}

yl)phosphinyl)[2.2]paracyclophane (25). To a cooled $\left(-67 \quad{ }^{\circ} \mathrm{C}\right)$ solution of bisphosphine $(R)-10(0.10 \mathrm{~g}, 0.15 \mathrm{mmol})$ in $\mathrm{CHCl}_{3}(10 \mathrm{~mL})$ was added a solution of hydroperoxide $17(0.030 \mathrm{~g}, 0.15 \mathrm{mmol})$ in $\mathrm{CHCl}_{3}(5 \mathrm{~mL})$. The reaction mixture was stirred for one-half hour and concentrated in vacuo. Purification by flash chromatography (hexanes/EtOAc 19:1) yielded mono(phosphine oxide) $(R)$-25 (0.050 g, $48 \%)$ as a white solid. ${ }^{1} \mathrm{H}$ NMR $\left(500 \mathrm{MHz}, \mathrm{CDCl}_{3}\right) \delta 7.43(\mathrm{~d}, J=11.6,2 \mathrm{H}), 7.09-7.22$ $(\mathrm{m}, 8 \mathrm{H}), 7.03(\mathrm{~s}, 1 \mathrm{H}), 6.97(\mathrm{~s}, 1 \mathrm{H}), 6.89(\mathrm{~s}, 1 \mathrm{H}), 6.73(\mathrm{~d}, J=7.7,1 \mathrm{H}), 6.67,(\mathrm{dd}, J=7.6$, 4.1, 1H), 6.55-6.60 (m, 2H), 6.48-6.54 (m, 1H), 3.10-3.14 (m, 3H), 2.91-3.00 (m, 3H), 2.50-2.78 (m, 2H), $2.41(\mathrm{~s}, 6 \mathrm{H}), 2.36(\mathrm{~s}, 6 \mathrm{H}), 2.25(\mathrm{~s}, 6 \mathrm{H}), 2.21(\mathrm{~s}, 6 \mathrm{H}) ;{ }^{13} \mathrm{C}$ NMR $(125$ $\left.\mathrm{MHz} \mathrm{CDCl}_{3}\right) \delta 146.0(\mathrm{~d}, J=7.5), 142.8(\mathrm{~d}, J=12.5), 139.2(\mathrm{~d}, J=2.5), 139.2(\mathrm{~d}, J=$ 13.8), 139.0, 138.9, 138.8, 137.7, $137.6(\mathrm{~d}, J=11.3), 137.53,137.51,137.45(\mathrm{~d}, J=2.5)$, $137.1(\mathrm{~d}, J=7.5), 136.9$ (d, $J=101.3), 136.8(\mathrm{~d}, J=2.5), 136.7(\mathrm{~d}, J=8.8), 135.2(\mathrm{~d}, J$ $=11.3), 134.2(\mathrm{~d}, J=3.8), 134.1(\mathrm{~d}, J=3.8), 133.5(\mathrm{~d}, J=2.5), 133.18(\mathrm{~d}, J=2.5)$, $133.17(\mathrm{~d}, J=21.2), 132.8(\mathrm{~d}, J=2.5) 131.5(\mathrm{~d}, J=26.3), 131.1(\mathrm{~d}, J=21.3) 130.5$, $129.8(\mathrm{~d}, J=7.5), 129.7,36.0(\mathrm{~d}, J=7.5), 35.0,32.7(\mathrm{~d}, J=3.8), 21.6 ;{ }^{31} \mathrm{P}$ NMR $(162$ $\mathrm{MHz}, \mathrm{CDCl}_{3}$ ) $\delta 21,-2$; IR (thin film) 3316, 2859, 1454, 1265, 1183, $1068 \mathrm{~cm}^{-1}$; HRMS (ESI) $\mathrm{m} / \mathrm{z}$ calcd for $\mathrm{C}_{48} \mathrm{H}_{50} \mathrm{OP}_{2}[\mathrm{M}+\mathrm{H}]^{+} 721.3364$, found 721.3369 .

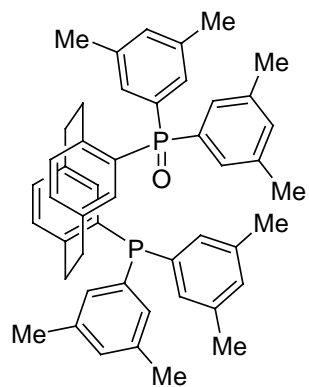

$(R)-25$

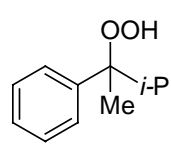

$( \pm)-11$

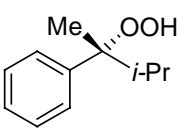

$(+)-(R)-11$

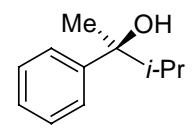

$(-)-(S)-12$

Scheme 3, Entry 1: The representative procedure was followed using compound 11 (11 $\mathrm{mg}, 0.060 \mathrm{mmol})$ and compound $(R)-25(18 \mathrm{mg}, 0.025 \mathrm{mmol})$. The reaction mixture was filtered through a plug of silica $\left(100 \% \mathrm{CH}_{2} \mathrm{Cl}_{2}\right)$ yielding a mixture of hydroperoxide (+)$(R)-11$ and alcohol $(-)-(S)-12(15 \mathrm{mg})$. The conversion (0.51) and ee of recovered starting material $(81 \%)$ were determined by SFC [Chiracel OD-H column $(250 \mathrm{~mm} \times 4.6$ 
$\mathrm{mm}$ i.d.) with $\mathrm{MeOH}: \mathrm{CO}_{2}(1: 99 \mathrm{v} / \mathrm{v})$, flow rate $1.0 \mathrm{~mL} \cdot \mathrm{min}^{-1}$, detection by $\mathrm{UV}$ absorbance at $210 \mathrm{~nm}$, retention times: $(+)-(R)-\mathbf{1 1}=5.8 \mathrm{~min}$ (major) and $(-)-(S)-\mathbf{1 1}=8.4$ $\min$ (minor)] and correspond to a $k_{\text {rel }}$ of 25 . 


\section{Determination of Absolute Configuration and Rotation.}

The literature data regarding the absolute configuration and optical rotation of the compounds included in this communication are scattered and to facilitate the flow we have made extrapolations based on the available data. Kunath et. al. have reported HPLC traces of hydroperoxides 11, 13, 14 and 17 and the corresponding alcohols using a cellulose-tris(3,5-dimethylphenyl carbamate) solid support. ${ }^{34}$ This support is similar to Chiracel's OD and OD-H columns that we utilized in our research. In all cases the elution order was $(+)$-alcohol, $(-)$-alcohol, $(+)$-hydroperoxide, $(-)$-hydroperoxide. In addition, alcohols $\mathbf{2 8},{ }^{35} \mathbf{3 0},{ }^{35} \mathbf{3 4},{ }^{36} \mathbf{3 6},{ }^{10} \mathbf{4 1},{ }^{37} \mathbf{4 2},{ }^{38} \mathbf{4 6},{ }^{39} \mathbf{4 7},{ }^{40}$ are known in enantioenriched form and in all cases the $R$ enantiomers are dextrarotary $(+)$ and the $S$ enantiomers are levorotory (-). We surmise, for all benzylic compounds, the hydroperoxide enantiomer eluting first corresponds to the $R-(+)$ enantiomer and the second peak corresponds to the $S-(-)$ enantiomer.

Table 3. Absolute stereochemistry and Optical Rotation Data

\begin{tabular}{|c|c|c|c|c|c|}
\hline entry & $\mathrm{R}^{1}$ & $\mathrm{R}^{2}$ & substrate & $\begin{array}{l}\mathrm{ROH} \text { and } \mathrm{ROOH} \\
\text { elution order }\end{array}$ & $\begin{array}{c}\text { absolute } \\
\text { configuration }^{b}\end{array}$ \\
\hline 1 & $\mathrm{Me}$ & $\mathrm{Et}$ & 42 & $1^{\text {st }}-(+), 2^{\text {nd }}-(-)$ & $(S)-(-)$ \\
\hline 2 & $\mathrm{Me}$ & $i-\mathrm{Pr}$ & 12 & $1^{\text {st }}-(+), 2^{\text {nd }}-(-)$ & $(R)-(+)$ \\
\hline 3 & $\mathrm{Me}$ & $n-\operatorname{Pr}$ & 28 & $1^{\text {st }}-(+), 2^{\text {nd }}-(-)$ & $(S)-(-)$ \\
\hline 4 & $\mathrm{Me}$ & $n-\mathrm{Bu}$ & 30 & $n d^{c}$ & $(S)-(-)$ \\
\hline 5 & $\mathrm{Me}$ & $i-\mathrm{Bu}$ & 32 & $\mathrm{nd}^{c}$ & $\mathrm{nd}^{c}$ \\
\hline 6 & $\mathrm{Me}$ & $c-\mathrm{C}_{6} \mathrm{H}_{11}$ & 34 & $1^{\text {st }}-(+), 2^{\text {nd }}-(-)$ & $(R)-(+)$ \\
\hline 7 & $\mathrm{H}$ & $\mathrm{Me}$ & 46 & $1^{\text {st }}-(+), 2^{\text {nd }}-(-)$ & $(R)-(+)$ \\
\hline 8 & $\mathrm{H}$ & Et & 47 & $\mathrm{nd}^{c}$ & $(R)-(+)$ \\
\hline 9 & $\mathrm{H}$ & $i-\operatorname{Pr}$ & 38 & $\mathrm{nd}^{c}$ & $(R)-(+)$ \\
\hline 10 & $\mathrm{H}$ & $c-\mathrm{C}_{6} \mathrm{H}_{11}$ & 41 & $\mathrm{nd}^{c}$ & $(S)-(-)$ \\
\hline
\end{tabular}


1. Pangborn, A. B.; Giardello, M. A.; Grubbs, R. H.; Rosen, R. K.; Trimmers, F. J. Organometallics 1996, 15, 1518-1520.

2. Rappoport, Z., The Chemistry of Peroxides. Wiley: Chichester, 2006

3. See note 6 in: Hiatt, R. R.; Strachan, W. M. J. Org. Chem. 1963, 28, 1893-1894.

4. The procedure employed was adapted from the work of Rapoport: Jamison, T. F.;

Lubell, W. D.; Dener, J. M.; Kriche, M. J.; Rapoport, H. Org. Syn. 1993, 71, 220.

5. Fukuzawa, S.; Mutoh, K.; Tsuchimoto, T.; Hiyama, T. J. Org. Chem. 1996, 61, 5400-5405.

6. Khalilov, L. M.; Parfenova, L. V.; Pechatkina, S. V.; Ibragimov, A. G.; Genet, J. P.; Dzhemilev, U. M.; Beletskaya, I. P. J. Organomet. Chem. 2004, 689, 444-453.

7. Ramon, D. J.; Yus, M. Tetrahedron 1998, 54, 5651-5666.

8. Kropf, H.; Helmut, V. W. J. Chem. Res. Synop. 1983, 11, 282-283.

9. Curran, D. P.; Totleben, M. J. J. Am. Chem. Soc. 1992, 114, 6050-6058.

10. Yang, W. K.; Cho, B. T. Tetrahedron: Asymmetry 2000, 11, 2947-2953.

11. Wipf, P.; Aslan, D. C. J. Org. Chem. 2001, 66, 337-343.

12. Bogert, M. T.; Davidson, D. J. Am. Chem. Soc. 1934, 56, 185-190.

13. Keh, C. C. K.; Wei, C.; Li, C. J. J. Am. Chem. Soc. 2003, 125, 4062-4063.

14. Davies, A. G.; Foster, R. V.; White, A. M. J. Chem. Soc. 1953, 5, 1541-1547.

15. Tokuyasu, T.; Kunikawa, S.; McCullough, K. J.; Masuyama, A.; Nojima, M. J. Org. Chem. 2005, 70, 251-260.

16. Davies, A. G. J. Chem. Soc. 1962, 11, 4288-4290.

17. Höft, E.; Hamann, H.-J.; Kunath, A. J. prakt. Chem. 1994, 336, 534-537.

18. Chen, S. T.; Fang, J. M. J. Org. Chem. 1997, 62, 4349-4357.

19. Hiatt, R. R.; Strachan, W. M. J. J. Org. Chem. 1963, 28, 1893-1894.

20. Umbreit, M. A.; Sharpless, K. B. J. Am. Chem. Soc. 1977, 99, (5526-5528).

21. The procedure employed was adapted from the work of Davies: Davies, A. G.;

Foster, R. V.; White, A. M. J. Chem. Soc. 1953, 5, 1541-1547.

22. Sugamoto, K.; Matsushita, Y.; Matsui, T. J. Chem. Soc., Perkin Trans. 1 1998, 7 , 3989-3998.

23. Adam, W.; Hoch, U.; Lazarus, M.; Saha-Möller, C.; Schreier, P. J. Am. Chem. Soc. 1995, 117, 11898-11901.

24. Chirko, A. I. J. Org. Chem. USSR 1969, 3, 26-30.

25. Borch, R. F.; Valente, R. R. J. Med. Chem 1991, 34, 3052-3058.

26. Mashima, K.; Kusano, K. H.; Sato, N.; Matsumura, Y. I.; Nozaki, K.;

Kumobayashi, H.; Sayo, N.; Hori, Y.; Ishizaki, T.; Akutagawa, S.; Takaya, H. J. Org.

Chem. 1994, 59, 3064-3076.

27. Wu, H.; Yu, J.; Spencer, J. Org. Lett. 2004, 6, 4675-4678.

28. Pye, P. J.; Rossen, K.; Reamer, R. A.; Tsou, N. N.; Volante, R. P.; Reider, P. J. J. Am. Chem. Soc. 1997, 119, 6207-6208.

29. Pye, P. J.; Rossen, K.; Volante, R. P.; Co., M. Novel chiral bisphosphines. WO 97/47632, 1997.

30. Procedure adapted from the work of Pye: Pye, P. J.; Rossen, K.; Reamer, R. A.; Tsou, N. N.; Volante, R. P.; Reider, P. J. J. Am. Chem. Soc. 1997, 119, 6207-6208.

31. Kagan, H. B.; Fiaud, J. C., Topics in Stereochemistry. Wiley: New York, 1988; pp 249-330. 
32. Tokuyasu, T.; Kunikawa, S.; McCullough, K. J.; Masuyama, A.; Nojima, M. J. Org. Chem. 2005, 70, 251-260.

33. Garcia, C.; Walsh, P. J. Org. Lett. 2003, 5, 3641-3644.

34. Kunath, A.; Höft, E.; Hamann, H.-J. J. Chromatogr. 1991, 588, 352-355.

35. Sakuraba, H.; Ushiki, S. Tetrahedron Lett. 1990, 31, 5349-5352.

36. Dosa, P. I.; Fu, G. C. J. Am. Chem. Soc. 1998, 120, 445-446.

37. Langer, F.; Schwink, L.; Devasagayaraj, A.; Chavant, P. Y.; Knochel, P. J. Org. Chem. 1996, 61, 8229-8243.

38. Lynch, J. E.; Eliel, E. L. J. Am. Chem. Soc. 1984, 106, 2943-2948.

39. Hayashi, T.; Matsumura, Y.-I. Tetrahedron: Asymmetry 1991, 2, 601-612.

40. Nakamura, K.; Matsuda, T. J. Org. Chem. 1998, 63, 8957-8964. 

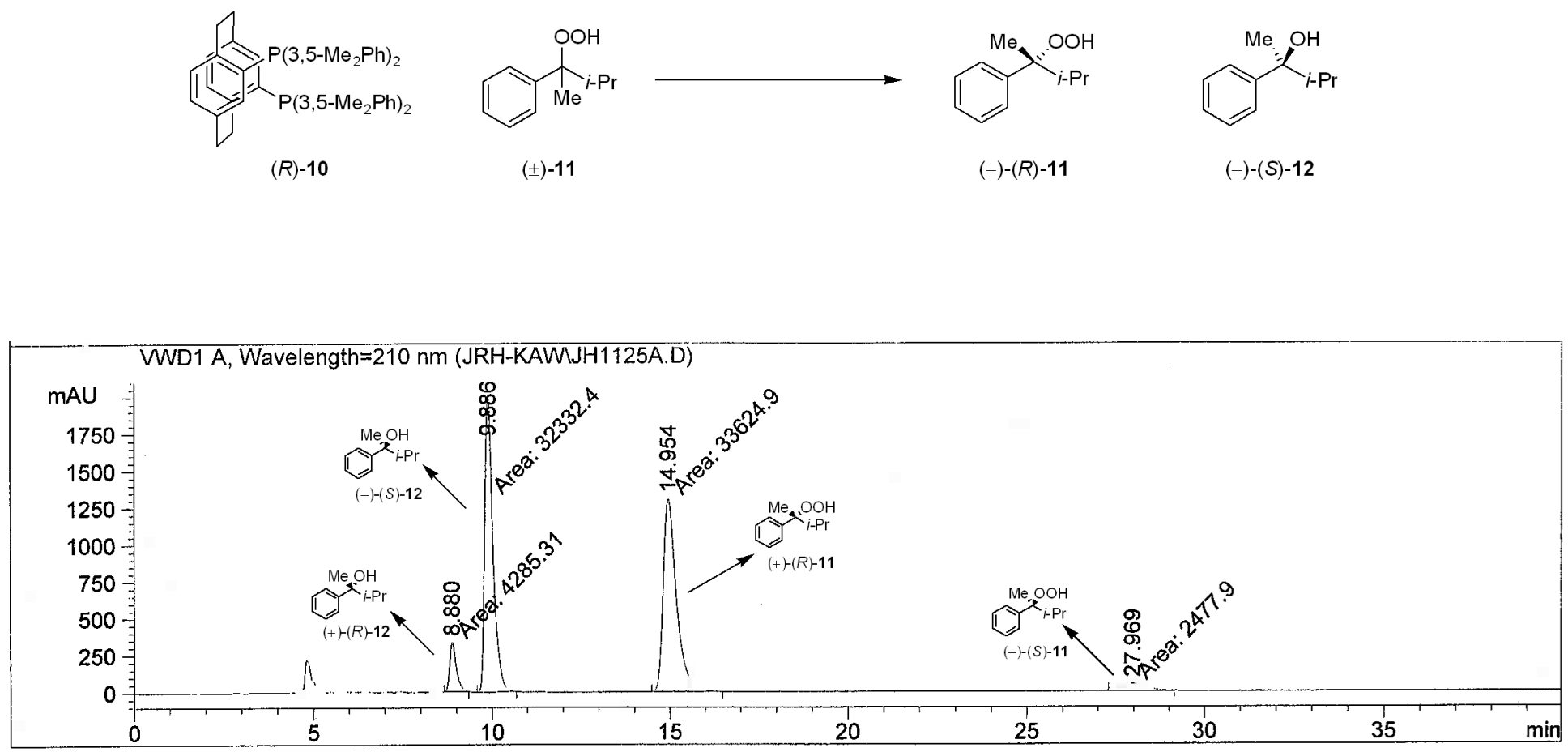

Signal 2: VWD1 A, Wavelength=210 nm

\begin{tabular}{|c|c|c|c|c|c|c|}
\hline $\begin{array}{c}\text { Peak } \\
\text { \# }\end{array}$ & $\begin{array}{c}\text { RetTime } \\
\text { [min] }\end{array}$ & Type & $\begin{array}{l}\text { Width } \\
\text { [min] }\end{array}$ & ${ }_{\mathrm{mAU}}^{\text {Area }}{ }^{*}{ }_{\mathrm{S}}$ & $\begin{array}{l}\text { Height } \\
{[\mathrm{mAU}]}\end{array}$ & $\begin{array}{c}\text { Area } \\
\frac{\circ}{0}\end{array}$ \\
\hline $\begin{array}{l}1 \\
2 \\
3 \\
4\end{array}$ & $\begin{array}{r}8.880 \\
9.886 \\
14.954 \\
27.969\end{array}$ & $\begin{array}{l}\text { MM } \\
\text { MM } \\
\text { MM } \\
\text { MM }\end{array}$ & $\begin{array}{l}0.2147 \\
0.2683 \\
0.4303 \\
0.8147\end{array}$ & $\begin{array}{l}4285.30762 \\
3.23324 \mathrm{e} 4 \\
3.36249 \mathrm{e} 4 \\
2477.90112\end{array}$ & $\begin{array}{r}332.63324 \\
2008.20044 \\
1302.41113 \\
50.69005\end{array}$ & $\begin{array}{r}5.8928 \\
44.4612 \\
46.2386 \\
3.4074\end{array}$ \\
\hline & & & & $7.2720 .6 \mathrm{e} 4$ & 3693.93486 & \\
\hline
\end{tabular}




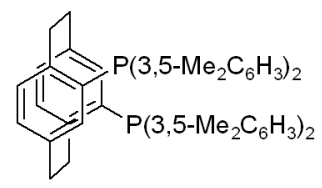

$(R)-10$<smiles>O=C([Mg])c1ccccc1</smiles>

$( \pm)-13$

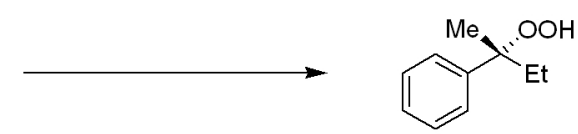

$(+)-(R)-13$

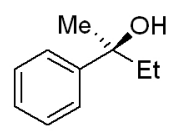

$(-)-(S)-42$

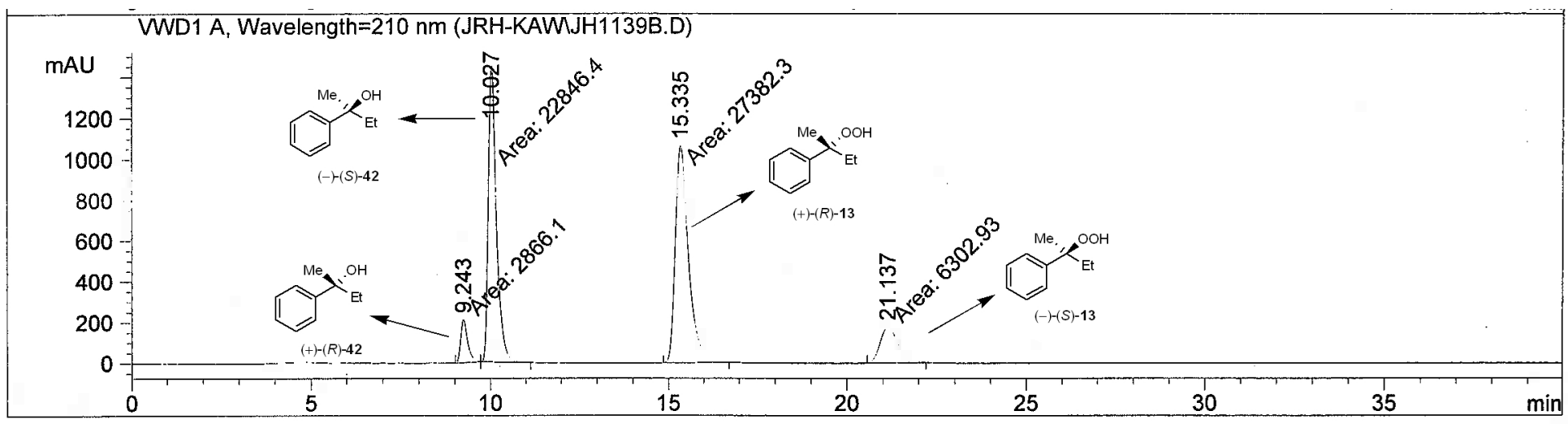

Signal 2: VWD1 A, Wavelength=210 nm

\begin{tabular}{|c|c|c|c|c|c|c|}
\hline Peak & RetTime & Type & Width & Area & Height & Area \\
\hline \# & [min] & & [min] & mAU ${ }^{*} \mathrm{~s}$ & {$[\mathrm{mAU} \quad]$} & $\frac{9}{8}$ \\
\hline 1 & 9.243 & MM & 0.2263 & 2866.09741 & 211.09280 & 253 \\
\hline 2 & 10.027 & MM & 0.2631 & $2.28464 e 4$ & 1447.07007 & 38.4634 \\
\hline 3 & 15.335 & $\mathrm{MM}$ & 0.4294 & $2.73823 e 4$ & 1062.78259 & 46.0999 \\
\hline 4 & 21.137 & MM & 0.5964 & 6302.93359 & 176.15292 & 10.6114 \\
\hline ot: & & & & $5.93978 e 4$ & 2897.09839 & \\
\hline
\end{tabular}




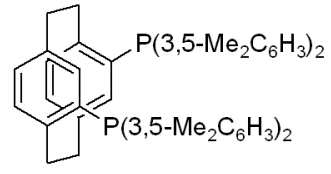

(S)-10

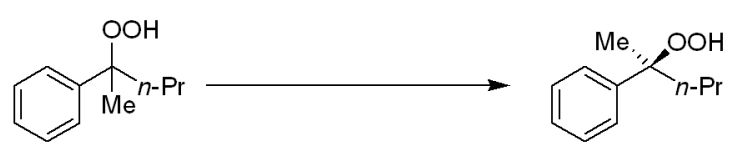

(土)-14<smiles>CCC(C)(O)c1ccccc1</smiles>

(+)-(R)-28

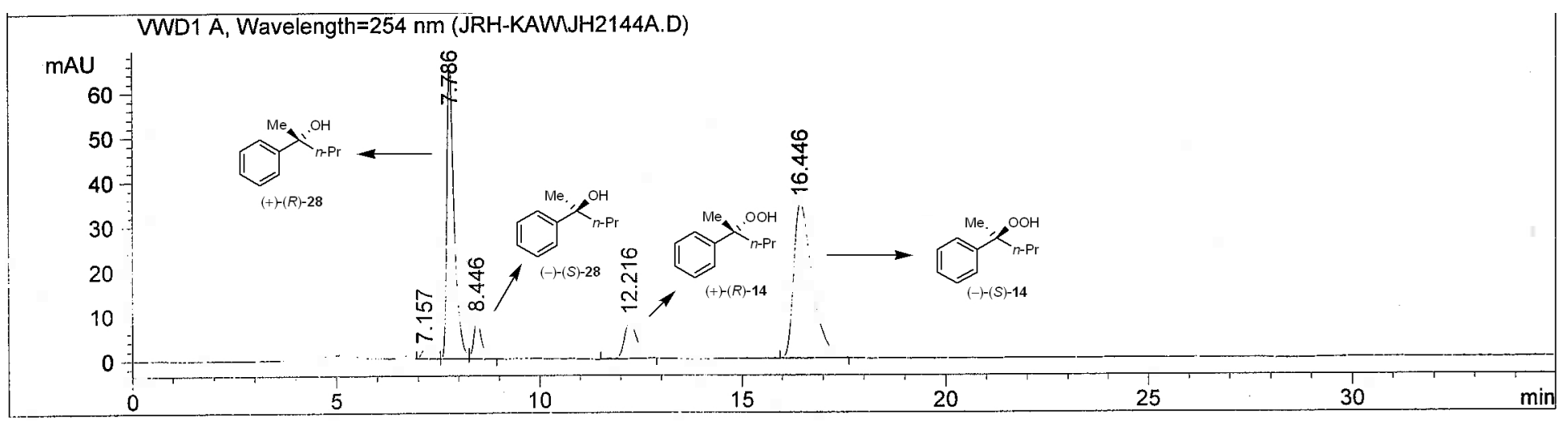

Signal 2: VWD1 A, Wavelength $=254 \mathrm{~nm}$

\begin{tabular}{|c|c|c|c|c|c|c|}
\hline Peak & RetTime & Type & Width & Area & Height & Area \\
\hline \# & [min] & & [min] & $\mathrm{mAU} \quad{ }^{*} \mathrm{~s}$ & {$[\mathrm{mAU} \quad]$} & \\
\hline 1 & 7.157 & VV & 0.2353 & 29.96621 & 1.97427 & 1.3254 \\
\hline 2 & 7.786 & VV & 0.1868 & 825.20050 & 65.93339 & 36.4976 \\
\hline 3 & 8.446 & VB & & 119.624 & & 908 \\
\hline 4 & 12 & $\mathrm{BB}$ & & 185.32 & & 8.1968 \\
\hline 5 & 16.446 & $\mathrm{BB}$ & $0.2+50$ & 1100.85571 & 34.65535 & 48.6895 \\
\hline & & & & 2260.97339 & 120.11080 & \\
\hline
\end{tabular}



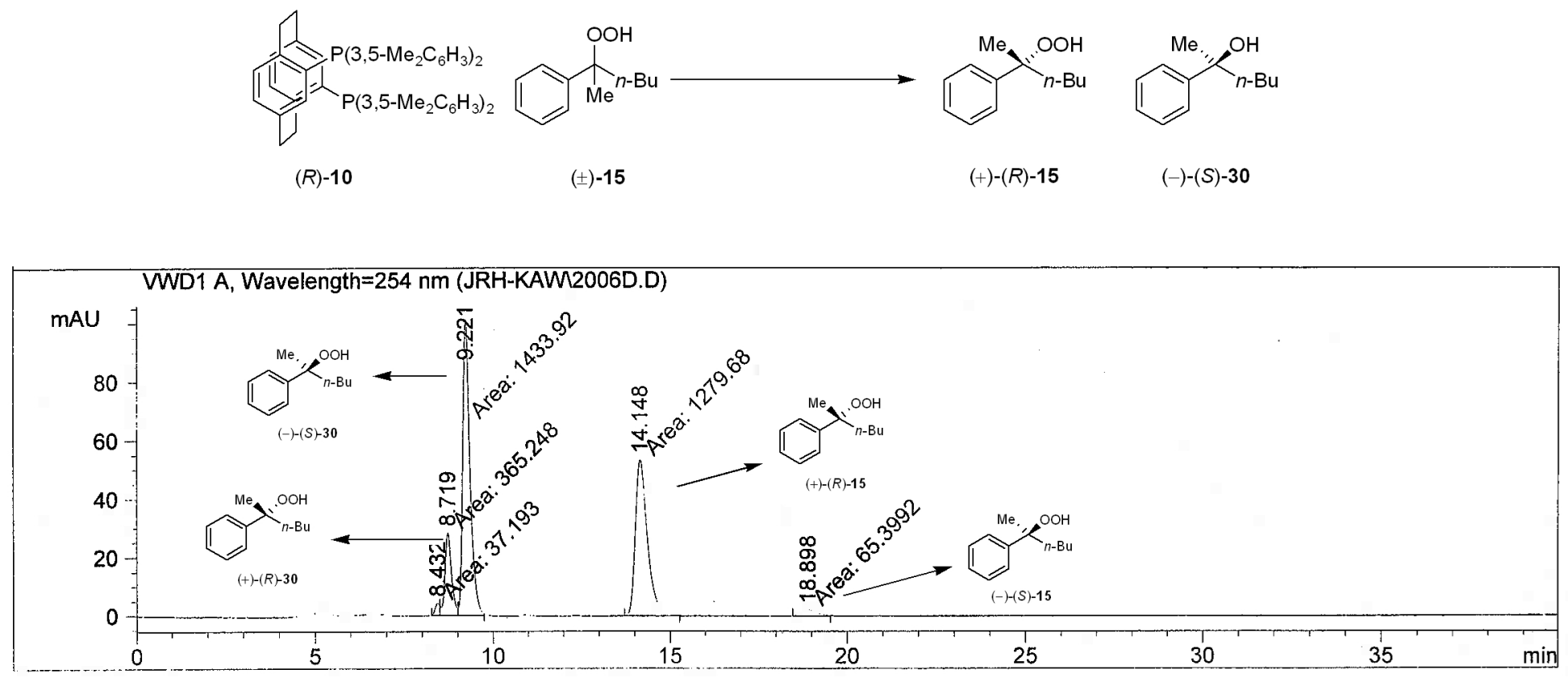

Signal 2: VWD1 A, Wavelength=254 nm

\begin{tabular}{|c|c|c|c|c|c|c|}
\hline \multirow{2}{*}{$\begin{array}{c}\text { Peak } \\
\#\end{array}$} & \multirow{2}{*}{$\begin{array}{c}\text { RetTime } \\
\text { [min] }\end{array}$} & \multirow[t]{2}{*}{ Type } & \multirow{2}{*}{$\begin{array}{l}\text { Width } \\
\text { [min] }\end{array}$} & Area & Height & \multirow{2}{*}{$\begin{array}{c}\text { Area } \\
\frac{\circ}{0}\end{array}$} \\
\hline & & & & $\mathrm{mAU} \quad *_{\mathrm{s}}$ & {$[\mathrm{mAU}]$} & \\
\hline & & & & ----------- & & 3 \\
\hline 1 & 8.432 & $\mathrm{MF}$ & 0.1461 & 37.19296 & 4.24358 & 1.1691 \\
\hline 2 & 8.719 & $\mathrm{ME}$ & 0.2154 & 365.24826 & 28.26128 & 11.4806 \\
\hline 3 & 9.221 & EM & 0.2364 & 1433.92200 & 101.10966 & 45.0714 \\
\hline 4 & 14.148 & MM & 0.3988 & 1279.68286 & 53.48181 & 40.2233 \\
\hline 5 & 18.898 & MM & 0.5082 & 65.39918 & 2.14486 & 2.0556 \\
\hline Tota & : & & & 3181.44525 & 189.24118 & \\
\hline
\end{tabular}




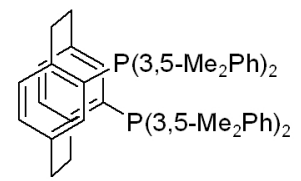

$(R)-10$<smiles>[R16]C(C)(C)c1ccccc1</smiles>

$( \pm)-16$<smiles>CC(C)(C)C(C)(C)O</smiles>

$(+)-(R)-16$

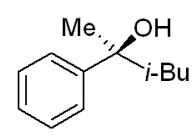

$(-)-(S)-32$

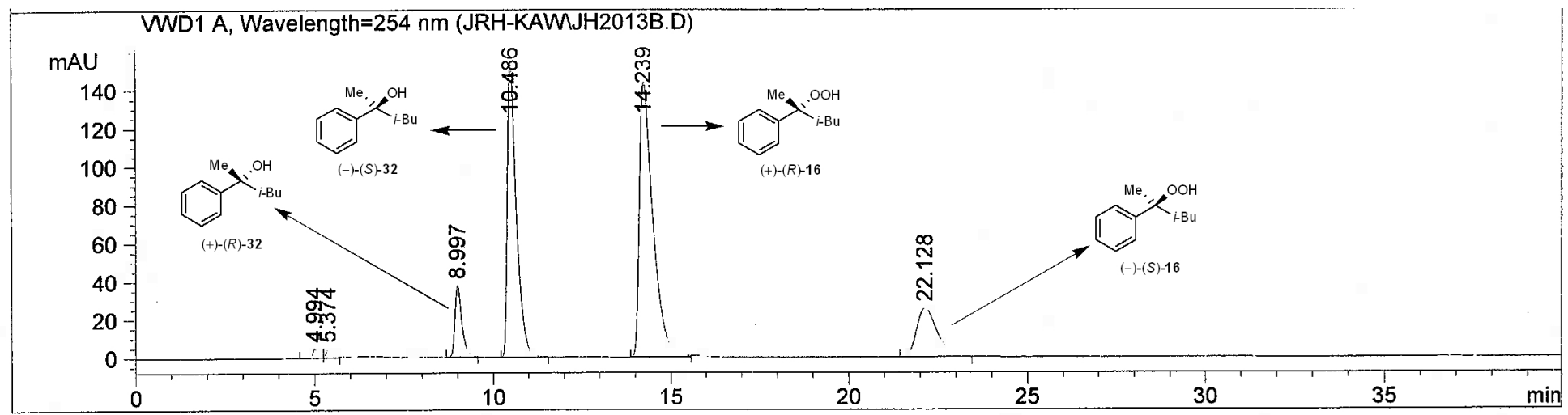

Signal 2: VWD1 A, Wavelength=254 nm

\begin{tabular}{|c|c|c|c|c|c|c|}
\hline \multirow{3}{*}{$\begin{array}{c}\text { Peak } \\
\#\end{array}$} & \multirow{2}{*}{$\begin{array}{c}\text { RetTime } \\
\text { [min] }\end{array}$} & \multirow[t]{2}{*}{ Type } & \multirow{2}{*}{$\begin{array}{l}\text { Width } \\
\text { [min] }\end{array}$} & Area & Height & \multirow{2}{*}{$\begin{array}{c}\text { Area } \\
\quad \frac{\circ}{\circ}\end{array}$} \\
\hline & & & & $\operatorname{mAU} \quad{ }^{\prime} \mathrm{s}$ & {$[\mathrm{mAU} \quad]$} & \\
\hline & -1 & & & $-0-1-1-1$ & -ーー・-ーーー & ------1 \\
\hline 1 & 4.994 & BV & 0.1664 & 62.20807 & 5.07065 & 0.7597 \\
\hline 2 & 5.374 & VB & 0.1605 & 57.15961 & 4.91371 & 0.6981 \\
\hline 3 & 8.997 & BB & 0.1993 & 505.36670 & 37.53416 & 6.1719 \\
\hline 4 & 10.486 & $\mathrm{BB}$ & 0.2580 & 2707.00610 & 154.74670 & 33.0598 \\
\hline 5 & 14.239 & BB & 0.3941 & 3831.74585 & 143.69745 & 46.7958 \\
\hline 6 & 22.128 & $\mathrm{BP}$ & 0.6080 & 1024.73376 & 25.61493 & 12.5147 \\
\hline Total & $:$ & & & 8188.22010 & 371.57760 & \\
\hline
\end{tabular}



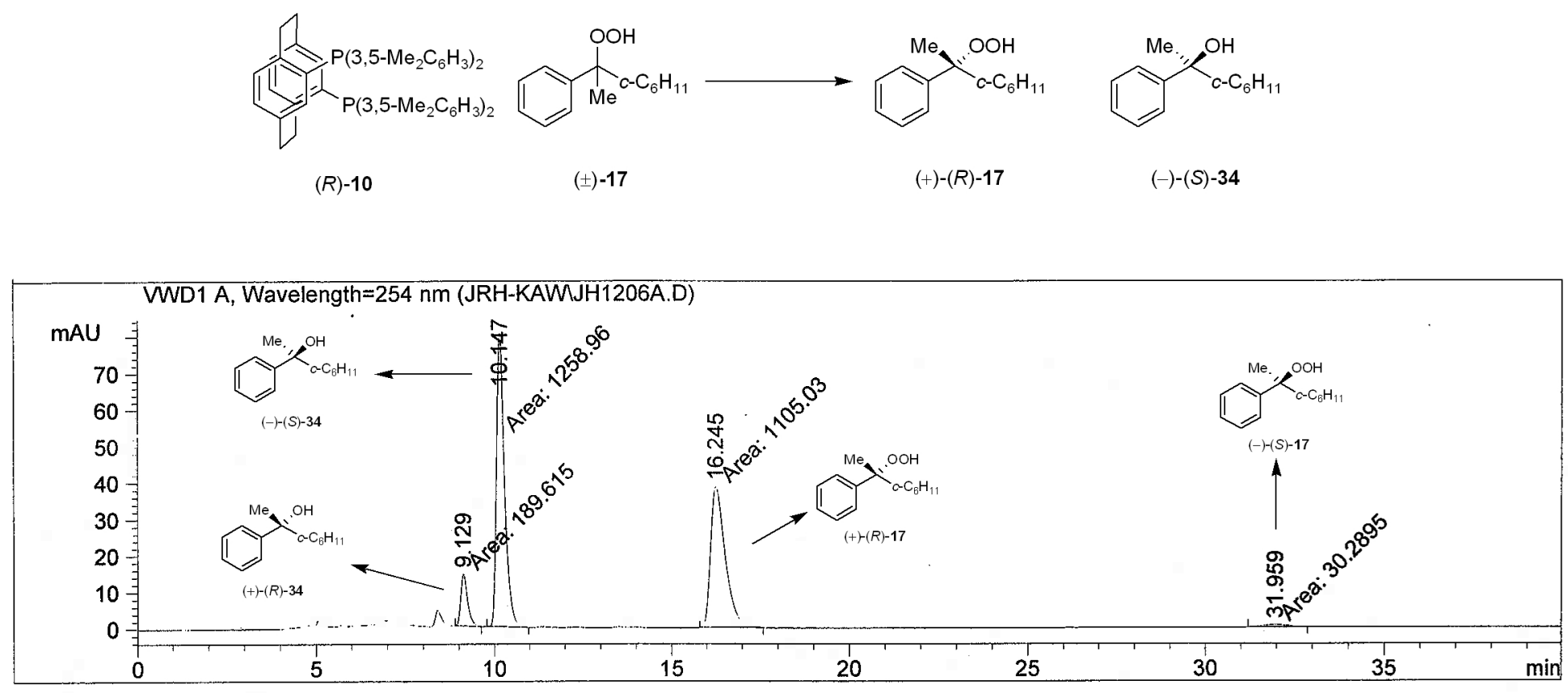

Signal 2: VWD1 A, Wavelength $=254 \mathrm{~nm}$

\begin{tabular}{|c|c|c|c|c|c|c|}
\hline Peak & RetTime & Type & Width & Area & Height & $\begin{array}{c}\text { Area } \\
\frac{\circ}{\partial}\end{array}$ \\
\hline \# & {$[\min ]$} & & {$[\min ]$} & mAU & {$[\mathrm{mAU} \quad]$} & \\
\hline 1 & $9: 129$ & MM & 0.2220 & 189.61459 & 14.23364 & 7.3383 \\
\hline 2 & 10.147 & MM & 0.2616 & 1258.96057 & 80.19722 & 48.7233 \\
\hline 3 & 16.245 & MM & 0.4773 & 1105.03162 & 38.58365 & 42.7661 \\
\hline 4 & 31.959 & MM & 0.8313 & 30.28949 & $6.07260 \mathrm{e}-1$ & 1.1722 \\
\hline Tota & : & & & 2583.89627 & 133.62177 & \\
\hline
\end{tabular}




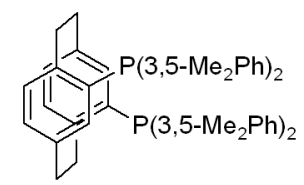

(R)-10

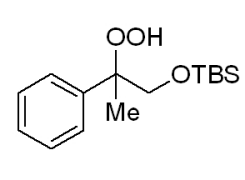

(土)-18

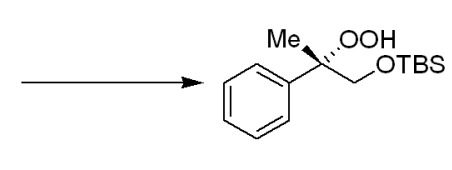

(+)- or (-)-18

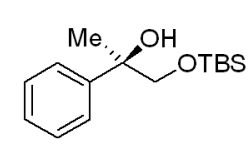

(+)- or (-)-54

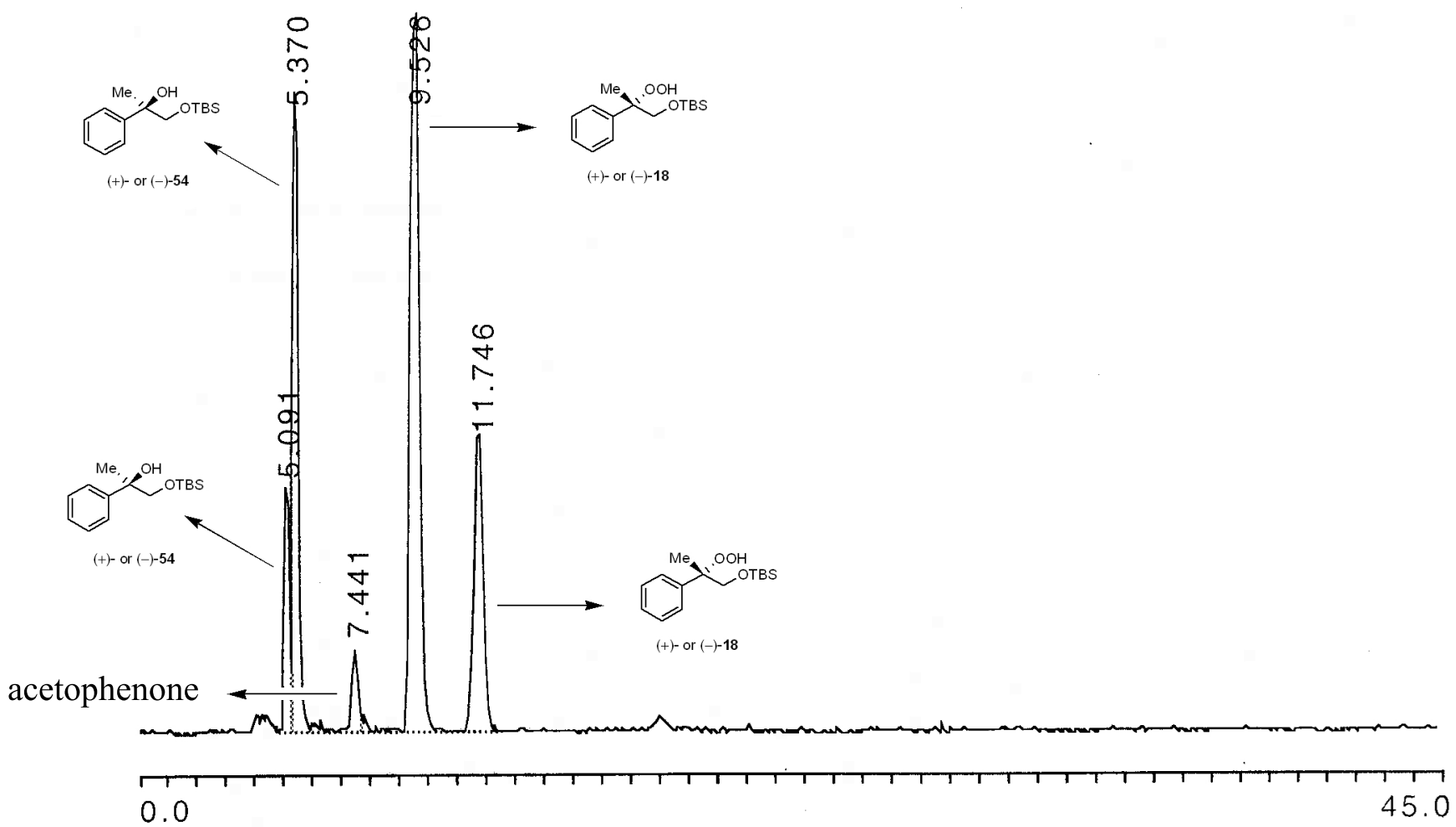

Analysis: Channel A

$\begin{array}{cr}\text { Peak No. } & \text { Time } \\ 1 & 5.091 \\ 2 & 5.370 \\ 3 & 7.441 \\ 4 & 9.526 \\ 5 & 11.746 \\ \text { Total Area } & \end{array}$

$\begin{array}{cr}\text { Type } & \text { Height }(\mu \mathrm{V}) \\ \text { N25 } & 618944 \\ \text { Err! } & 1608319 \\ \text { N34 } & 210244 \\ \text { Err! } & 1804918 \\ \text { N45 } & 748573\end{array}$

$\begin{array}{r}\text { Area }(\mu \mathrm{V} \text {-sec }) \\ 5439242 \\ 15854866 \\ 1985839 \\ 30877622 \\ 12662284 \\ -\frac{2}{6}-\frac{1}{1}-\frac{1}{6}-1 \\ \hline 6819853\end{array}$

Area $\%$

8.140

23.727

2.971

46.210

18.949

99.997 


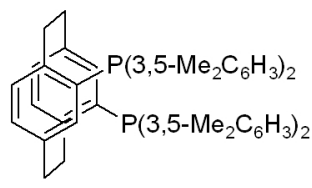

$(R)-10$

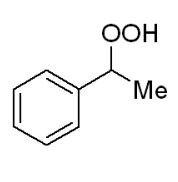

(士)-19

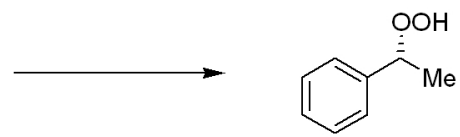

$(+)-(R)-19$

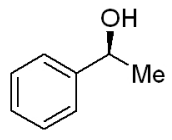

$(-)-(S)-46$

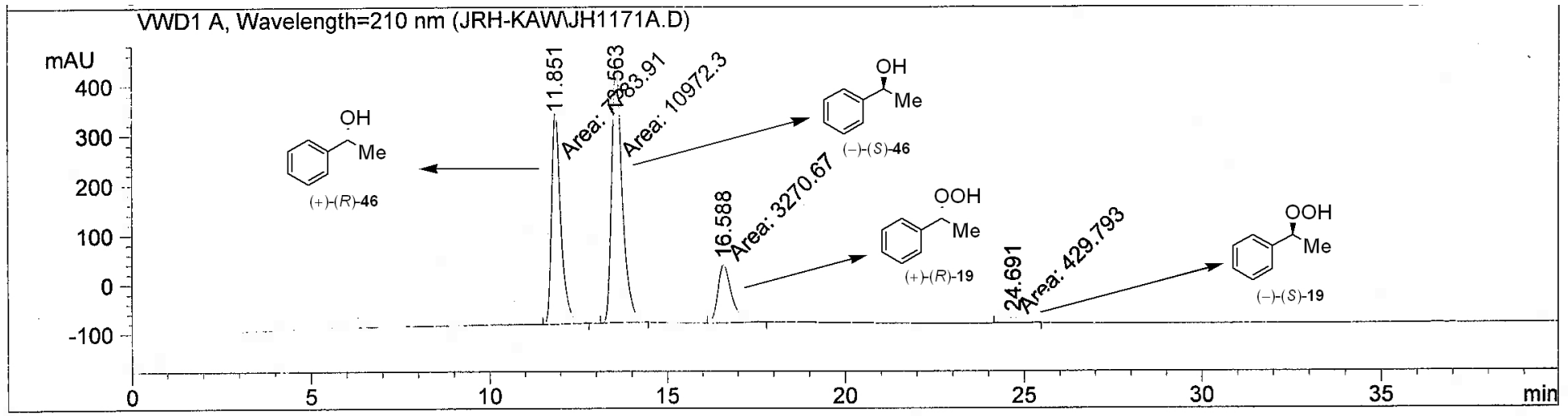

Signal 2: VWD1 A, Wavelength=210 nm

\begin{tabular}{|c|c|c|c|c|c|c|}
\hline eak & & $\mathrm{V}$ & Width & Area & Height & Area \\
\hline \# & & & & $\mathrm{mAU} \quad{ }^{\star} \mathrm{s}$ & {$[\mathrm{mAU} \quad]$} & 응 \\
\hline & & & & & & \\
\hline 1 & & MM & & 778 & 432 & \\
\hline 2 & & MM & 0.3446 & $1.09723 \mathrm{e} 4$ & 530. & \\
\hline 3 & 16.588 & MM & 0.4608 & 3270.66919 & 118.30054 & 14.5644 \\
\hline 4 & 24 & MM & 506 & 429.79272 & 11.01073 & 1.9139 \\
\hline
\end{tabular}

Totals :

$2.24567 \mathrm{e} 4 \quad 1092.03988$ 


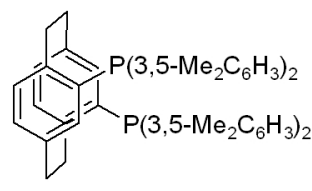

$(R)-10$<smiles>CCC(OO)c1ccccc1</smiles>

$( \pm)-20$<smiles>CC[C@H](O)c1ccccc1</smiles>

$(+)-(R)-20$<smiles>CC[C@H](O)c1ccccc1</smiles>

$(-)-(S)-47$

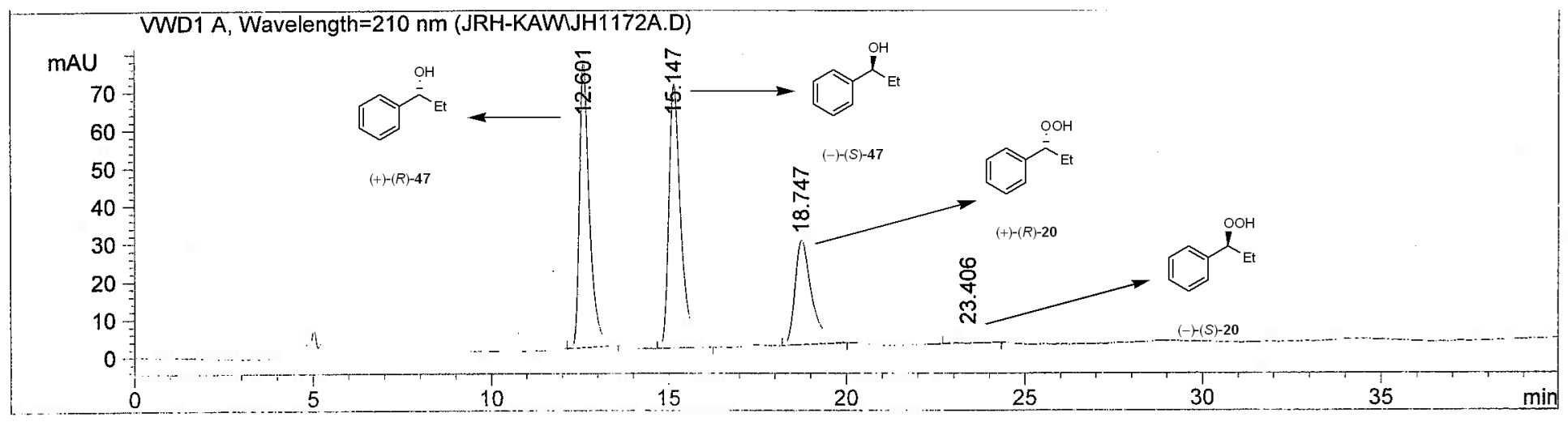

Signal 2: VWD1 A, Wavelength=210 nm

\begin{tabular}{|c|c|c|c|c|c|c|}
\hline Peak & RetTime & Type & Width & Area & Height & Area \\
\hline$\#$ & [min] & & [min] & $\mathrm{mAU} \quad{ }^{*} \mathrm{~s}$ & {$[\mathrm{mAU}]$} & 응 \\
\hline & --ー-ー-ー & & & & - - - - - - - & \\
\hline 1 & 12.601 & VP & 0.2903 & 1479.83167 & 75.34127 & 35.6759 \\
\hline 2 & 15.147 & $\mathrm{BP}$ & 0.3494 & 1635.34729 & 69.70401 & 39.4251 \\
\hline 3 & 18.747 & $\mathrm{PP}$ & 0.4881 & 911.40149 & 27.63652 & 21.9721 \\
\hline 4 & 23.406 & $B B$ & 0.5440 & 121.40751 & 3.34754 & 2.9269 \\
\hline Tota & & & & 4147.98795 & 176. & \\
\hline
\end{tabular}



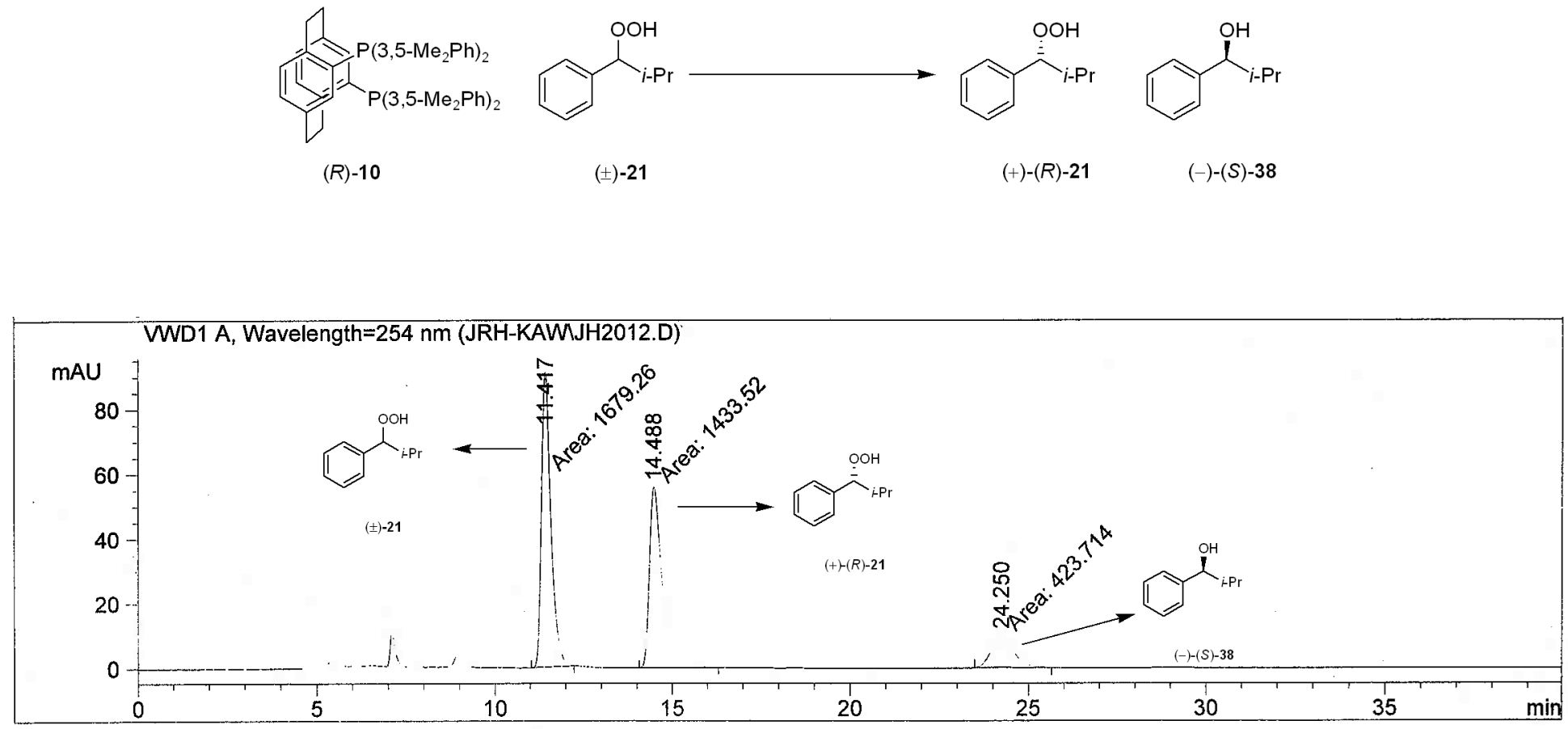

Signal 2: VWD1 A, Wavelength=254 nm

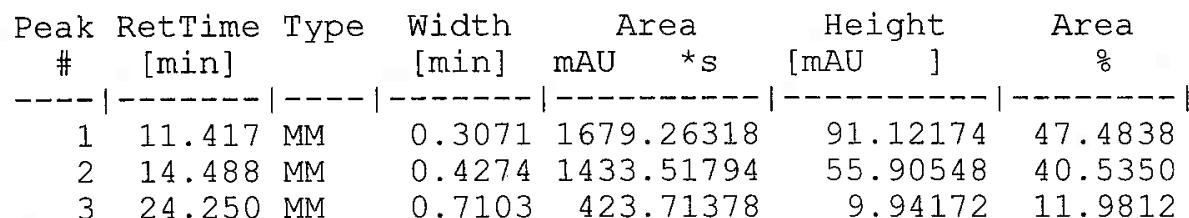

Totals :

$3536.49490 \quad 156.96894$ 

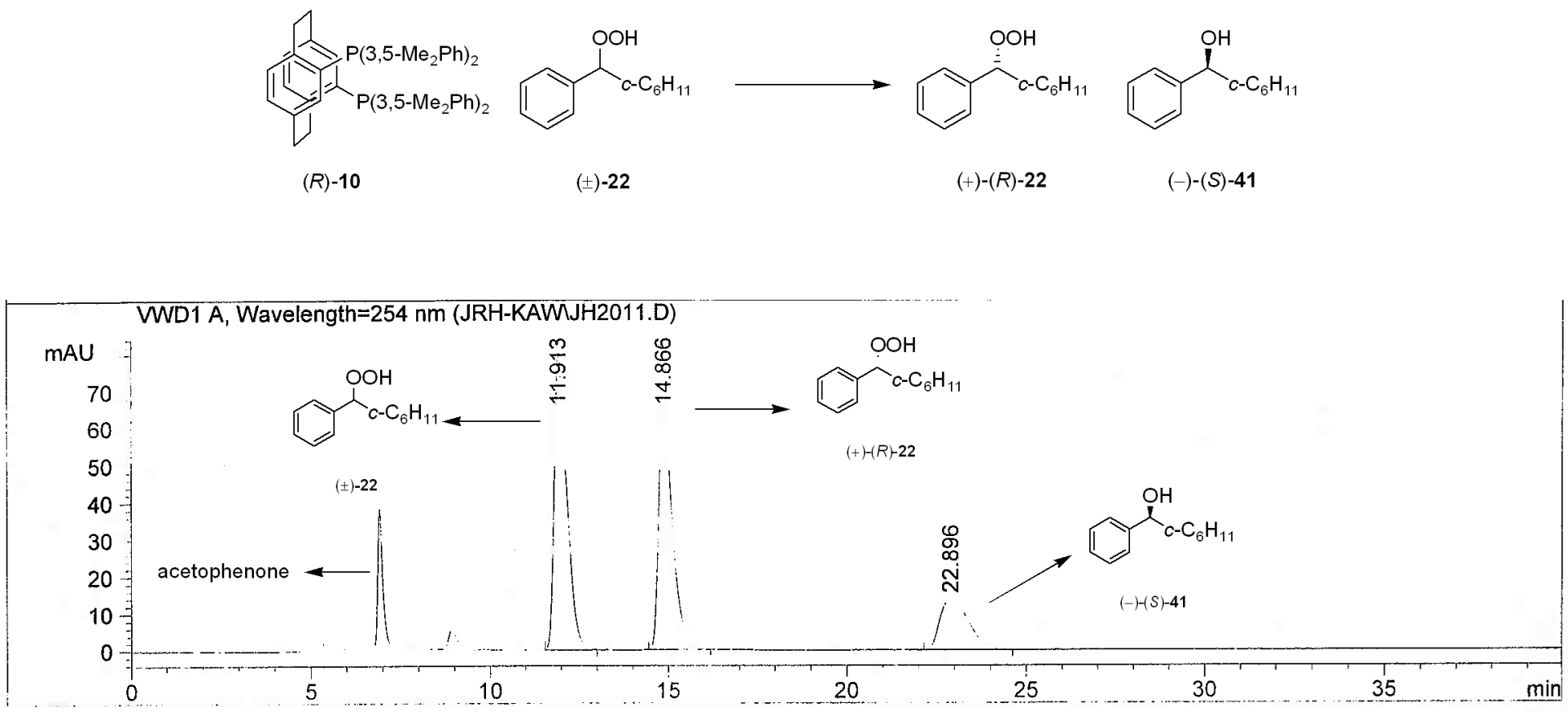

Signal 2: VWD1 A, Wavelength=254 nm

\begin{tabular}{|c|c|c|c|c|c|c|}
\hline Peak & RetTime & Type & Width & Area & Height & Area \\
\hline \# & [min] & & [min] & $\mathrm{mAU} \quad * s$ & {$[\mathrm{mAU} \quad]$} & 음 \\
\hline--- & & & & ----------1 & ---------- & $----\cdots$ \\
\hline 1 & 11.913 & VB & 0.3385 & 1941.12097 & 80.38700 & 43.1016 \\
\hline 2 & 14.866 & $\mathrm{BB}$ & 0.3947 & 1814.14124 & 68.55012 & 40.2821 \\
\hline 3 & 22.896 & $B B$ & 0.8965 & 748.32928 & 13.66760 & 6163 \\
\hline Tota & . & & & 4503.59149 & 162 & \\
\hline
\end{tabular}




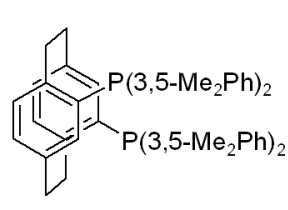

$(R)-10$

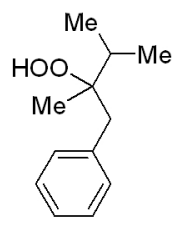

$( \pm)-23$

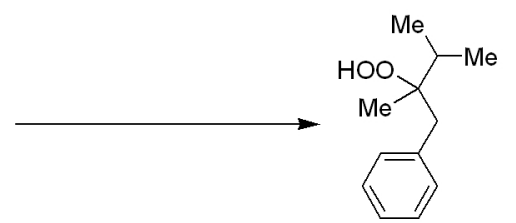

$(+)$ or $(-)-23$

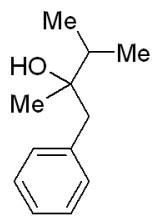

$(+)$ or $(-)-39$

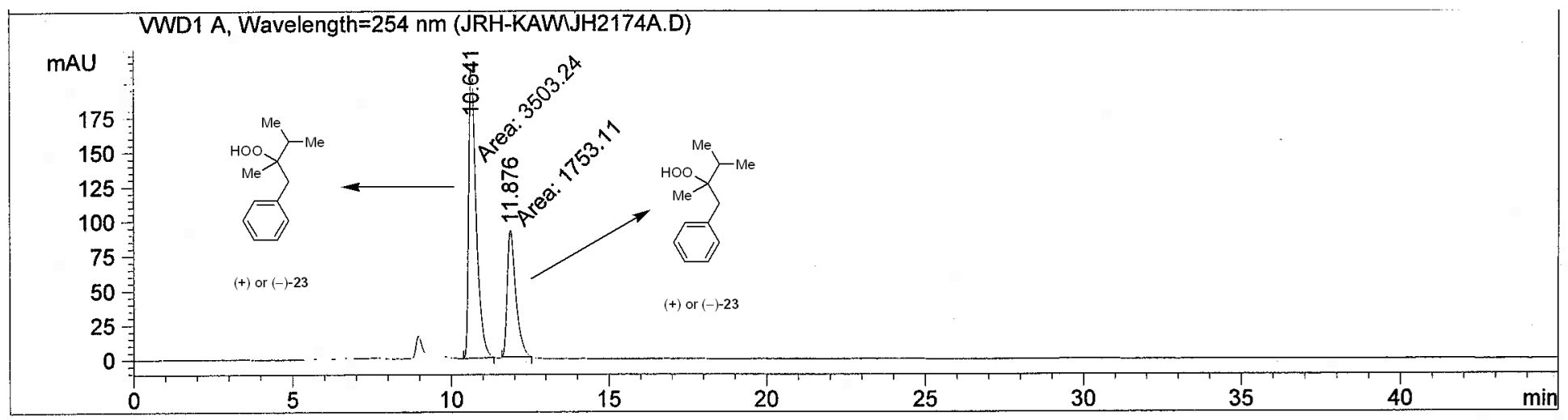

Signal 2: VWD1 A, Wavelength=254 nm

\begin{tabular}{|c|c|c|c|c|c|c|}
\hline \multirow{2}{*}{$\begin{array}{c}\text { Peak } \\
\#\end{array}$} & \multirow{2}{*}{$\begin{array}{l}\text { RetTime } \\
{[\mathrm{min}]}\end{array}$} & \multirow[t]{2}{*}{ Type } & \multirow{2}{*}{$\begin{array}{l}\text { Width } \\
\text { [min] }\end{array}$} & Area & Height & \multirow{2}{*}{$\begin{array}{c}\text { Area } \\
\frac{\%}{0}\end{array}$} \\
\hline & & & & $\mathrm{mAU} \quad{ }^{*} \mathrm{~s}$ & {$[\mathrm{mAU}$} & \\
\hline & -4--- & & & -ーーーーーーーー- & --ー-ー-ー-ー- & ---1 \\
\hline 1 & 10.641 & MM & 0.2737 & 3503.24292 & 213.30818 & 66.6478 \\
\hline 2 & 11.876 & MM & 0.3191 & 1753.10510 & 91.57590 & 33.3522 \\
\hline Tota & $:$ & & & 5256.34802 & 304.88408 & \\
\hline
\end{tabular}




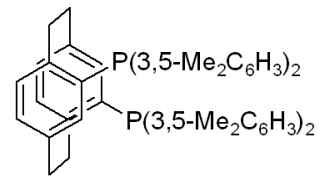

$(R)-10$<smiles>CC(O)(O)c1ccccc1</smiles>

$( \pm)-11$<smiles>CC(O)(P)c1ccccc1</smiles>

$(+)-(R)-11$

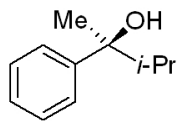

$(-)-(S)-12$

CCP1190.tmp.DAT - HP1100 DAD Signal A

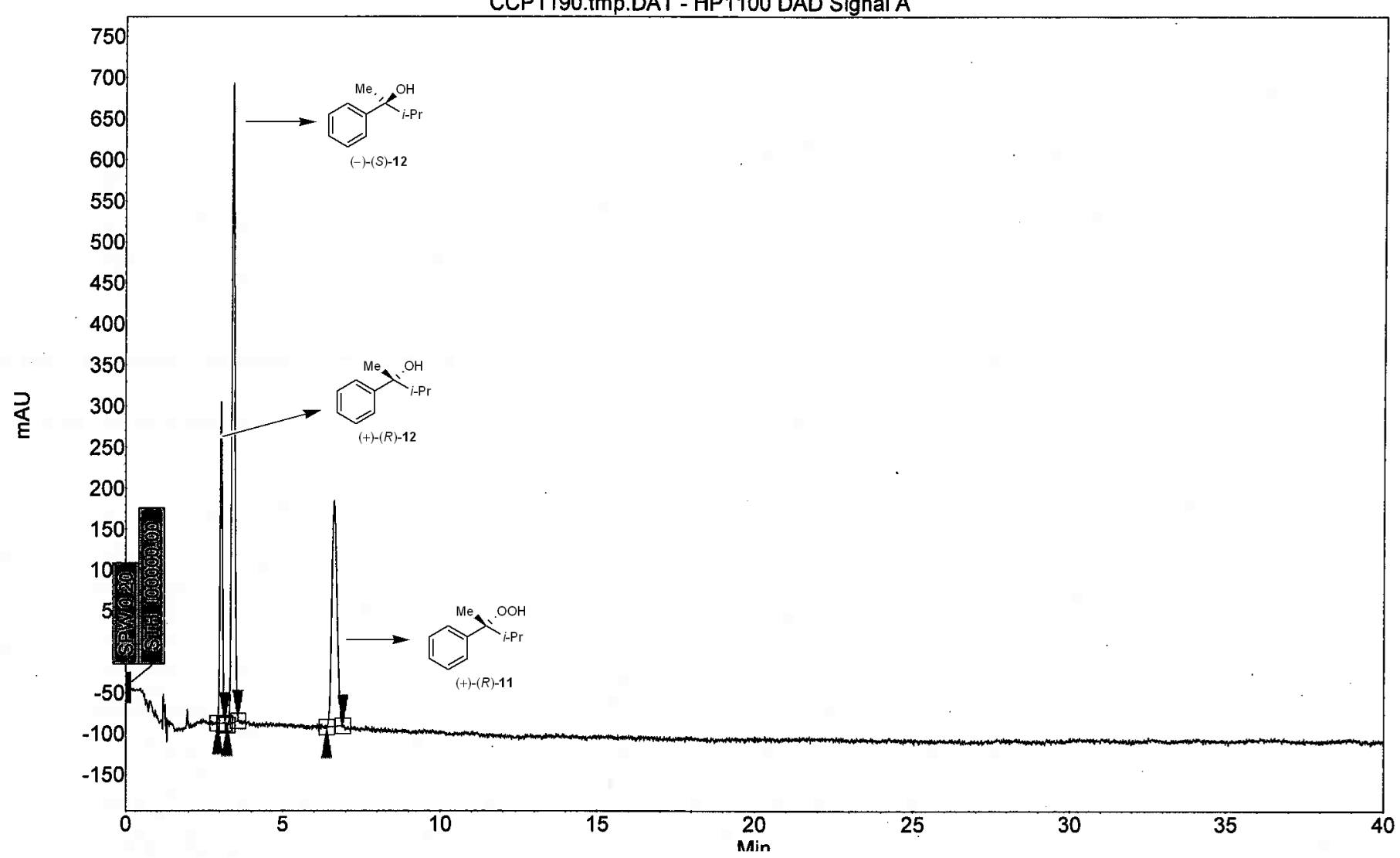

\begin{tabular}{|c|l|r|r|r|r|r|r|r|r|}
\hline Tindex & Name & Stant & Time & End & RT Offsel & Quantity & Height & Area & Area \\
\hline \hline & & {$[$ Min] } & {$[\mathrm{Min}]$} & {$[\mathrm{Min}]$} & {$[\mathrm{Min}]$} & {$[\%$ Area } & {$[\mu \mathrm{V}]$} & {$[\mu \mathrm{HV} . \mathrm{Min}]$} & {$[\%]$} \\
\hline 1 & UNKNOWN & 2.93 & 3.05 & 3.16 & 0.00 & 19.80 & 393.0 & 34.3 & 19.799 \\
\hline 2 & UNKNOWN & 3.24 & 3.42 & 3.58 & 0.00 & 49.91 & 780.0 & 86.5 & 49.910 \\
\hline 3 & UNKNOWN & 6.40 & 6.64 & 6.91 & 0.00 & 30.29 & 276.9 & 52.5 & 30.291 \\
\hline & & & & & & & & & \\
\hline Total & & & & & & 100.00 & 1449.8 & 173.4 & 100.000 \\
\hline
\end{tabular}




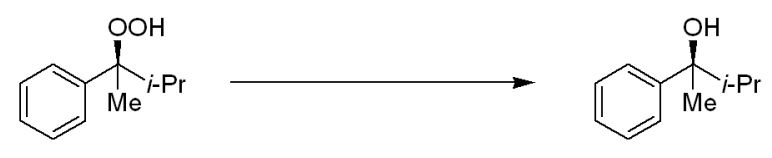
$(+)-(R)-11$
$(+)-(R)-12$

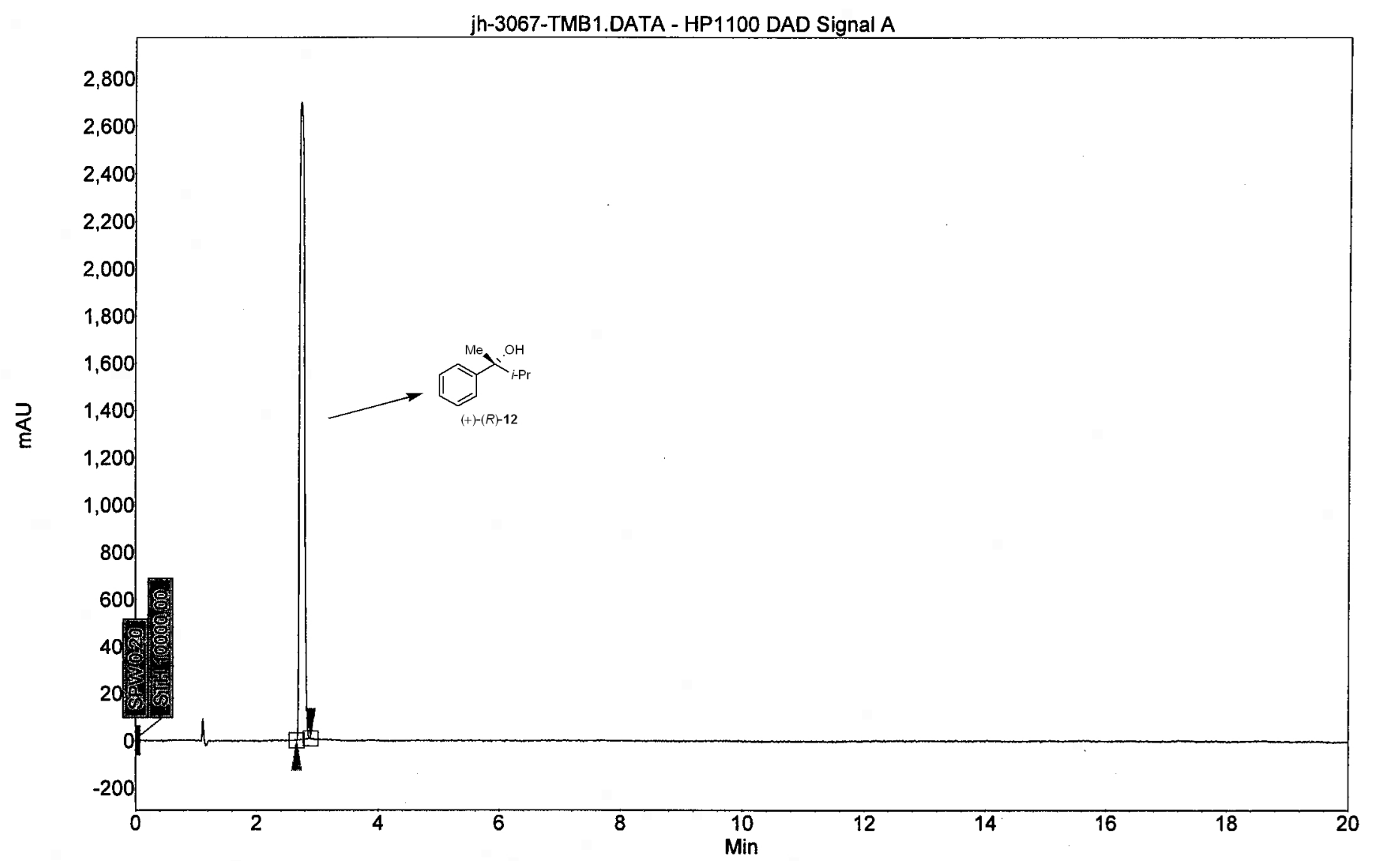

\begin{tabular}{|c|r|r|r|r|r|r|r|r|r|}
\hline \hline Index & Name & Stan & Time & End & RT Offset & Quantity & Height & Area & Area \\
\hline \hline & & {$[\mathrm{Min}]$} & {$[\mathrm{Min}]$} & {$[\mathrm{Min}]$} & {$[\mathrm{Min}]$} & {$[\%$ Area] } & {$[\mu \mathrm{N}]$} & {$[\mu \mathrm{V} . \mathrm{Min}]$} & {$[\%]$} \\
\hline 1 & UNKNOWN & 2.66 & 2.73 & 2.89 & 0.00 & 100.00 & 2695.5 & 249.2 & 100.000 \\
\hline & & & & & & & & & \\
\hline Total & & & & & & 100.00 & 2695.5 & 249.2 & 100.000 \\
\hline
\end{tabular}


<smiles>Cc1cc(C)cc(P(c2cc(C)cc(C)c2)c2ccccc2P(=O)(c2cc(C)cc(C)c2)c2cc(C)cc(C)c2)c1</smiles>

$(R)-25$<smiles>[Y16]C(O)(O)c1ccccc1</smiles>

$( \pm)-11$<smiles>CCCC(C)(C(=O)O)c1ccccc1</smiles>

$(+)-(R)-11$<smiles>CCCC(C)(O)c1ccccc1</smiles>

$(-)-(S)-12$

jh-3034-c1-cf11.DATA - HP1100 DAD Signal A

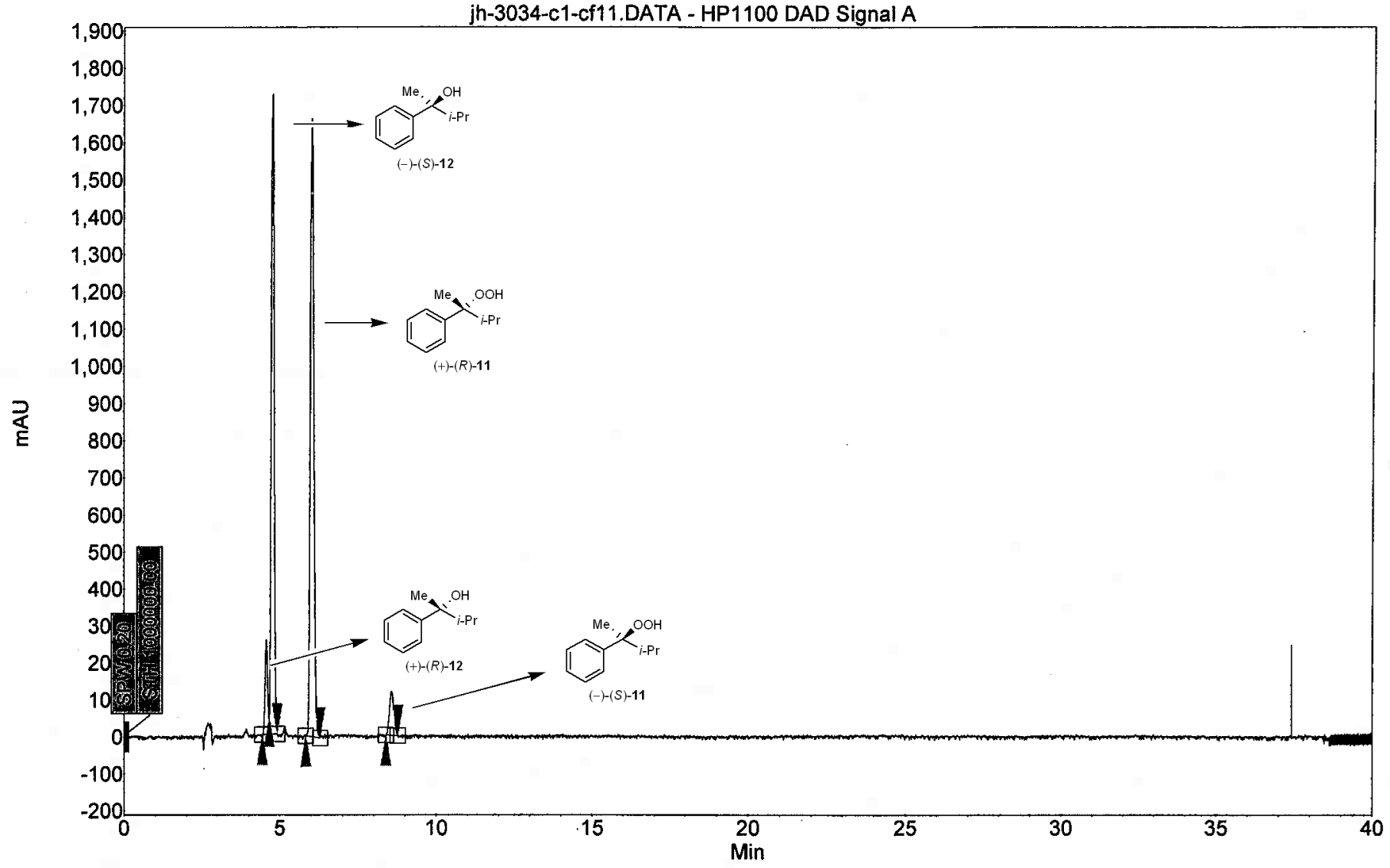

\begin{tabular}{|c|l|r|r|r|r|r|r|r|r|}
\hline Index & Name & Start & Time & End & RT Offset & Quantify & Height & Area & Area \\
\hline \hline & & {$[\mathrm{Min}]$} & {$[\mathrm{Min}]$} & {$[\mathrm{Min}]$} & {$[\mathrm{Min}]$} & {$[\%$ Area] } & {$[\mu \mathrm{V}]$} & {$[\mu \mathrm{V} . \mathrm{Min}]$} & {$[\%]$} \\
\hline 1 & UNKNOWN & 4.44 & 4.57 & 4.66 & 0.00 & 5.35 & 255.9 & 24.6 & 5.349 \\
\hline 4 & UNKNOWN & 4.66 & 4.76 & 4.92 & 0.00 & 41.48 & 1724.8 & 190.6 & 41.478 \\
\hline 2 & UNKNOWN & 5.82 & 6.02 & 6.29 & 0.00 & 48.82 & 1664.4 & 224.3 & 48.818 \\
\hline 3 & UNKNOWN & 8.40 & 8.57 & 8.77 & 0.00 & 4.36 & 119.1 & 20.0 & 4.355 \\
\hline & & & & & & & & & \\
\hline Total & & & & & & 100.00 & 3764.2 & 459.5 & 100.000 \\
\hline
\end{tabular}


$1 \mathrm{H}$ spectrum

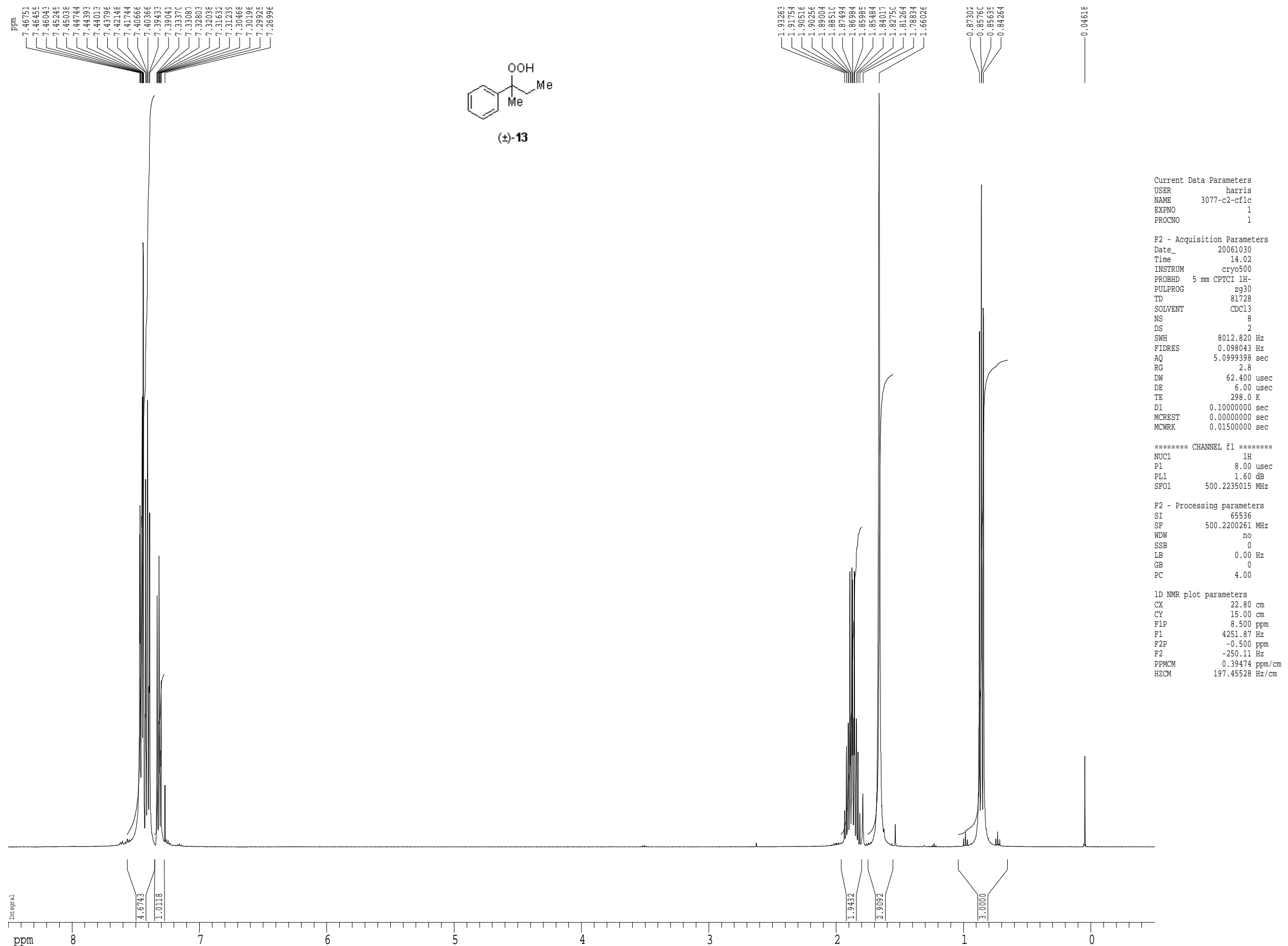


$13 \mathrm{C}$ spectrum with $1 \mathrm{H}$ decoupling

통

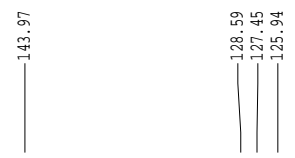

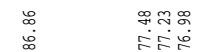

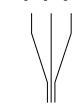

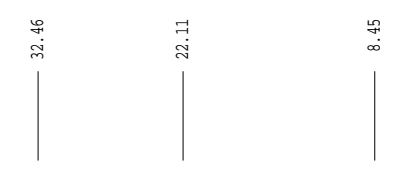

$\mathrm{OOH}$

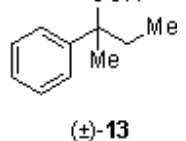

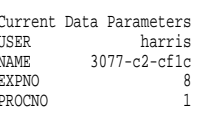

F2 - Acquisition Parameters

Date_ 20061030

Time
INSTRUM
PROBHD $\quad$ cryo5 $\mathrm{mm}$ CPTCI 14

PROBHD
PUIPROC
TD
SOLVEN

NS

$\begin{array}{lc}\text { DS } & 4 \\ \text { SWH } & 20161.291 \mathrm{~Hz} \\ \text { FIDRES } & 0.308286\end{array}$

$\begin{array}{lr}20161.291 \mathrm{~Hz} \\ \text { FIDRES } & 0.302866 \mathrm{~Hz} \\ \text { AQ } & 1.6219203 \mathrm{sec} \\ \text { PC } & 1459.5\end{array}$

$\begin{array}{lr}\text { RG } & 14596.5 \mathrm{ug} \\ \mathrm{RW} & 24.800 \mathrm{usec} \\ \mathrm{DE} & 6.00 \mathrm{usec} \\ \mathrm{TE} & 298.0 \mathrm{~K} \\ \mathrm{TE} & 0.2500000 \mathrm{sec}\end{array}$

$\begin{array}{ll}\text { D1 } & 0.25000000 \mathrm{sec} \\ \text { d11 } & 0.03000000 \mathrm{sec}\end{array}$

$\begin{array}{ll}\text { MCREST } & 0.000000000 \\ \text { MCWRK } & 0.01500000 \mathrm{sec}\end{array}$

$\begin{array}{ll}====== & \text { CHANNEL } \\ \text { f1 } 1 & ====== \\ \text { NUC1 } & 130 \\ \text { P1 } & 15,00 \text { usec }\end{array}$

$\begin{array}{lrl}\text { NUC1 } & 13 \mathrm{C} \\ \text { P1 } & 15.00 \text { usec } \\ \text { PL1 } & -1.00 \mathrm{~dB}\end{array}$

$=======$ CHANNEL $\mathrm{f} 2====== \pm$
WPDPRG2
waltz16

$\begin{array}{lc}\text { CPDPRG2 } & \text { waltz16 } \\ \text { NUC2 } & 1 \mathrm{H} \\ \text { PCD2 } & 100.00 \mathrm{usec} \\ \text { PL2 } & 1.60 \mathrm{~dB} \\ \text { PE2 } & 23.5 \mathrm{~g}\end{array}$

$\begin{array}{lr}\text { PLL2 } & \begin{array}{l}1.60 \mathrm{~dB} \\ \text { P12 }\end{array} \\ \text { SF02 } & 500.2225011 \mathrm{~dB} \\ \end{array}$

F2 - Processing parameters

$\begin{array}{ll}\text { SI } & 65536 \\ \text { SE } & 125.7804111 \mathrm{MHz}\end{array}$

WDW
SSB

GB
PC 1D NMR plot parameters
CX $22.80 \mathrm{~cm}$ $\begin{array}{lr}\text { CX } & 22.80 \mathrm{~cm} \\ \text { CY } & 15.65 \mathrm{~cm} \\ \text { F1P } & 160.000 \mathrm{ppm}\end{array}$ PPMCII 
$1 \mathrm{H}$ spectrum

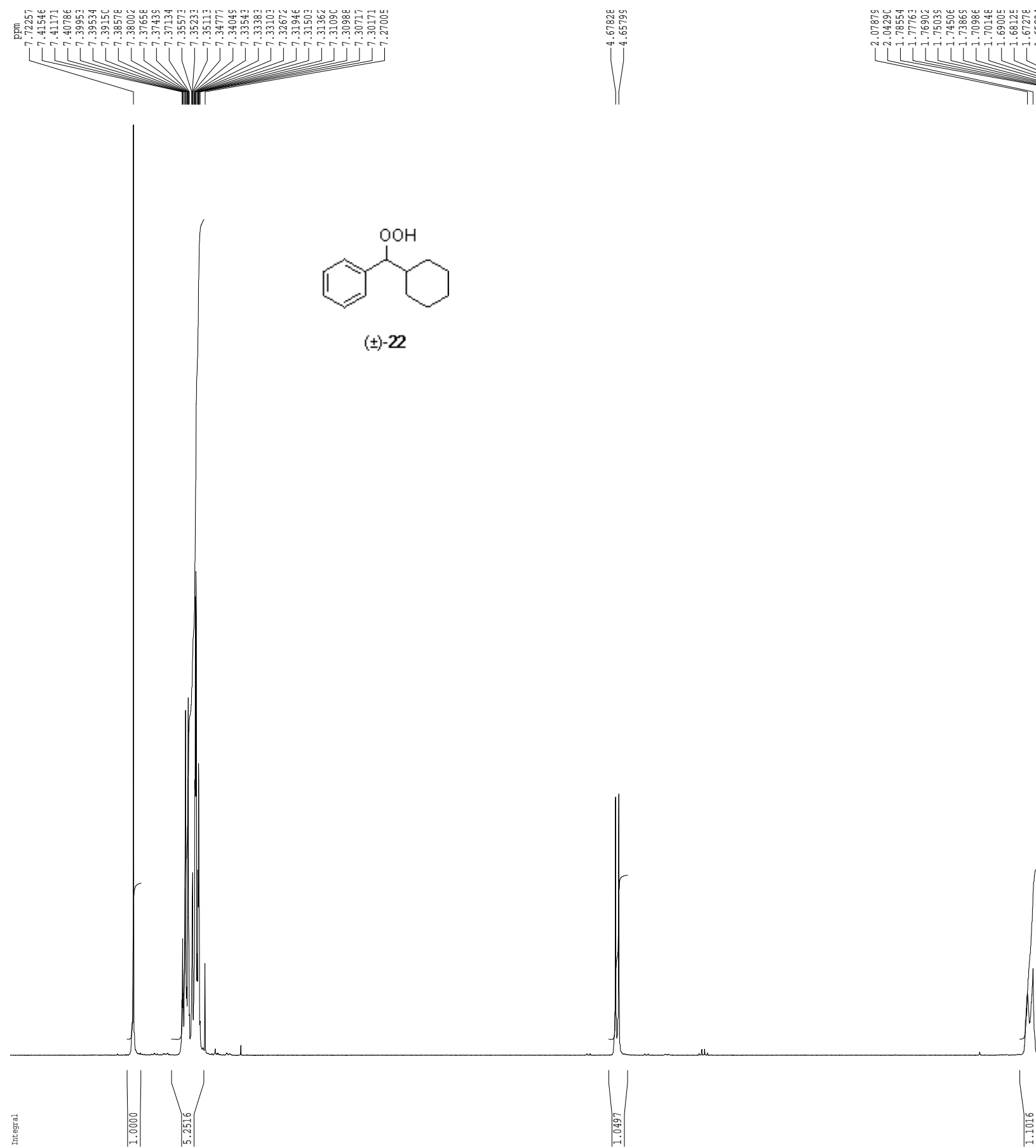

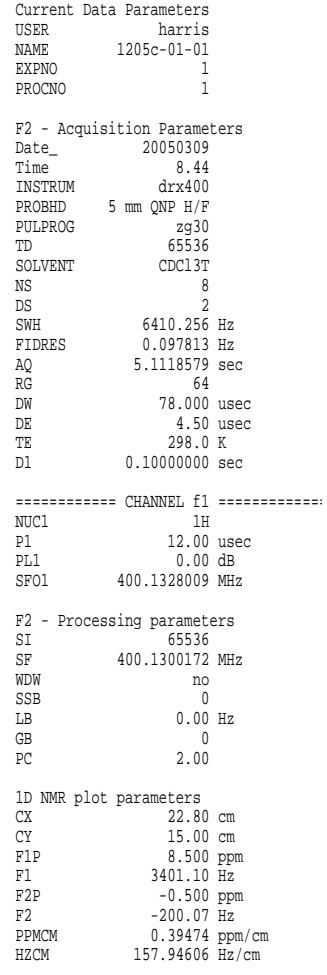


Supporting Information: Driver, Harris, Woerpel

$13 \mathrm{C}$ spectrum with $1 \mathrm{H}$ decoupling

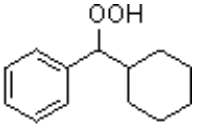

( \pm )-22

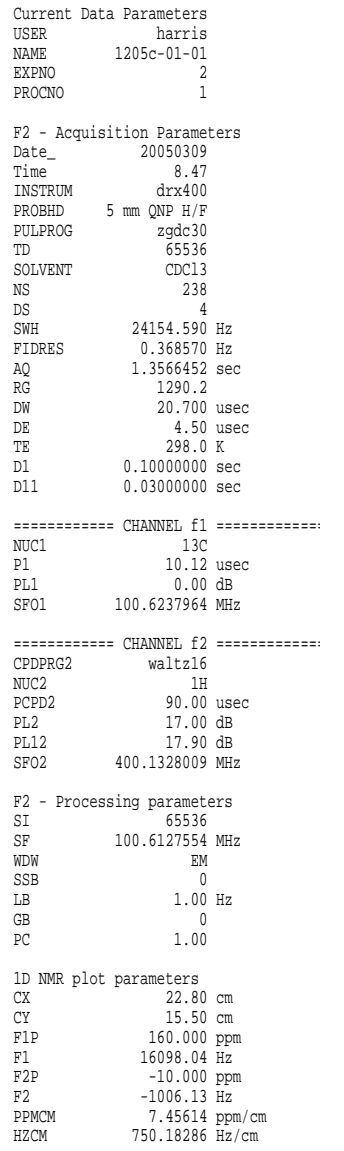


$1 \mathrm{H}$ spectrum

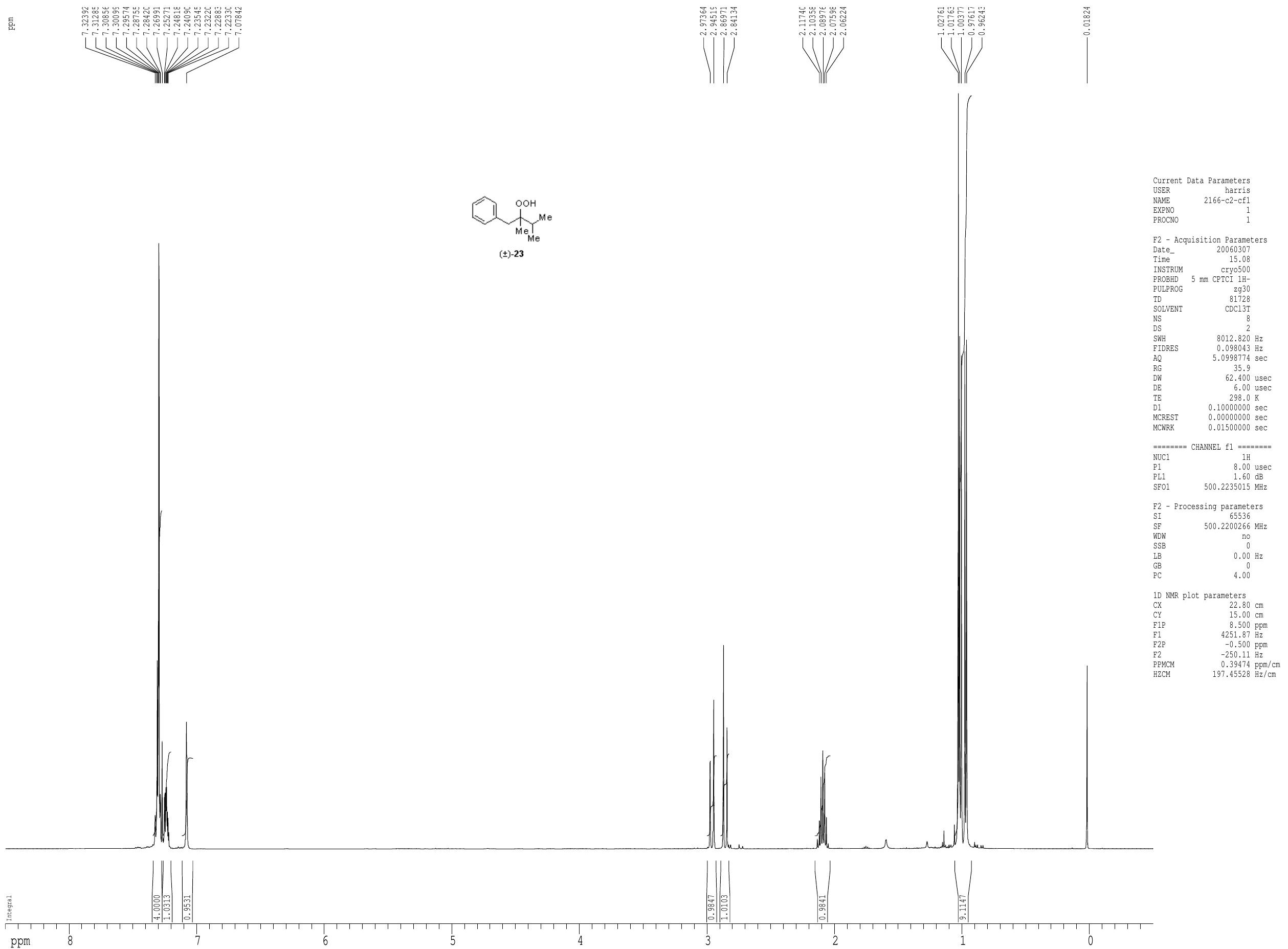


$13 \mathrm{C}$ spectrum with $1 \mathrm{H}$ decoupling
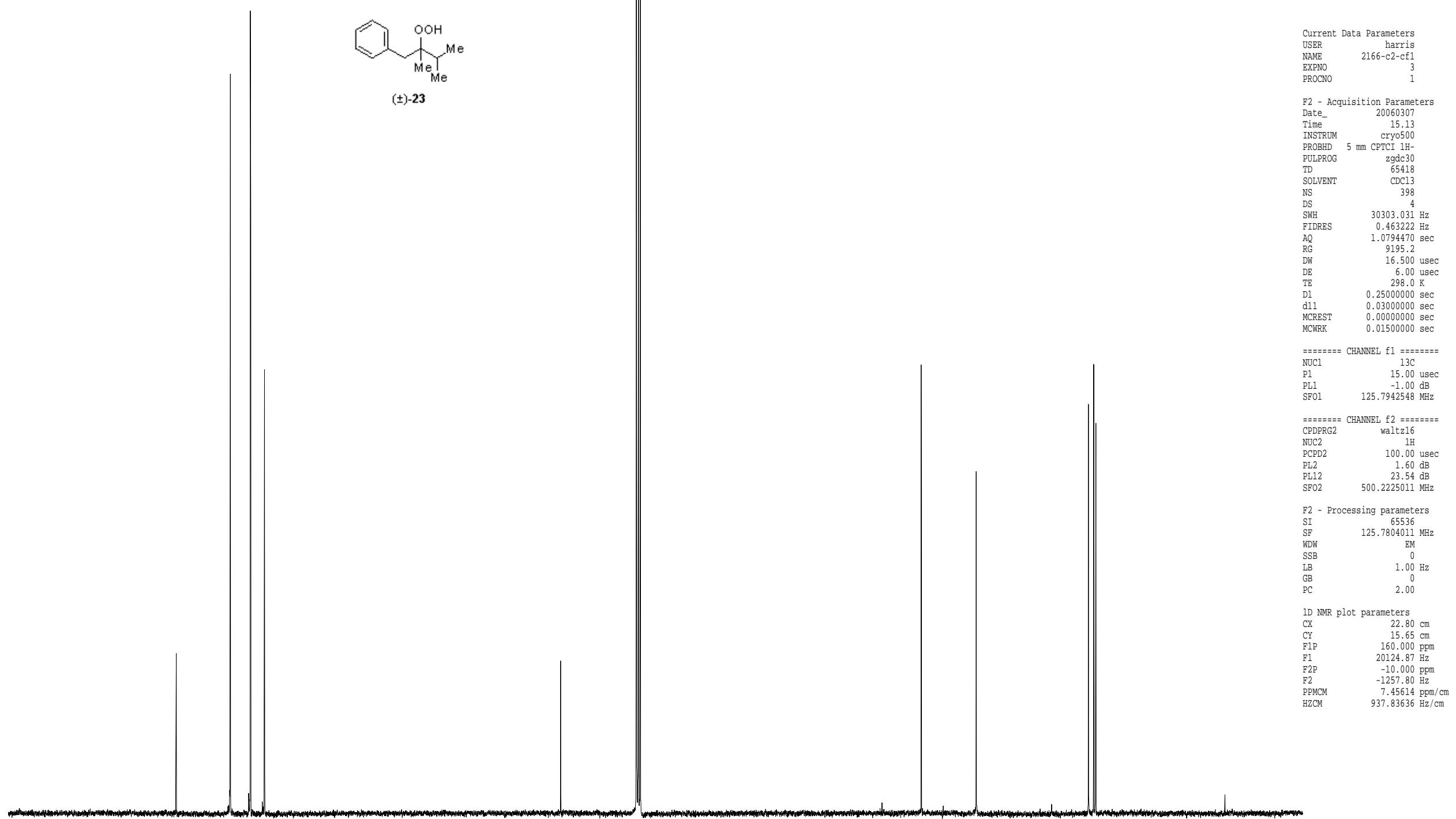
$1 \mathrm{H}$ spectrum

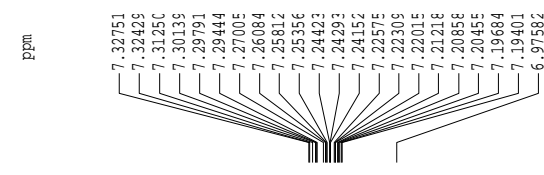

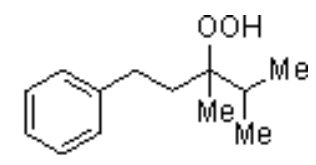

$( \pm)-24$
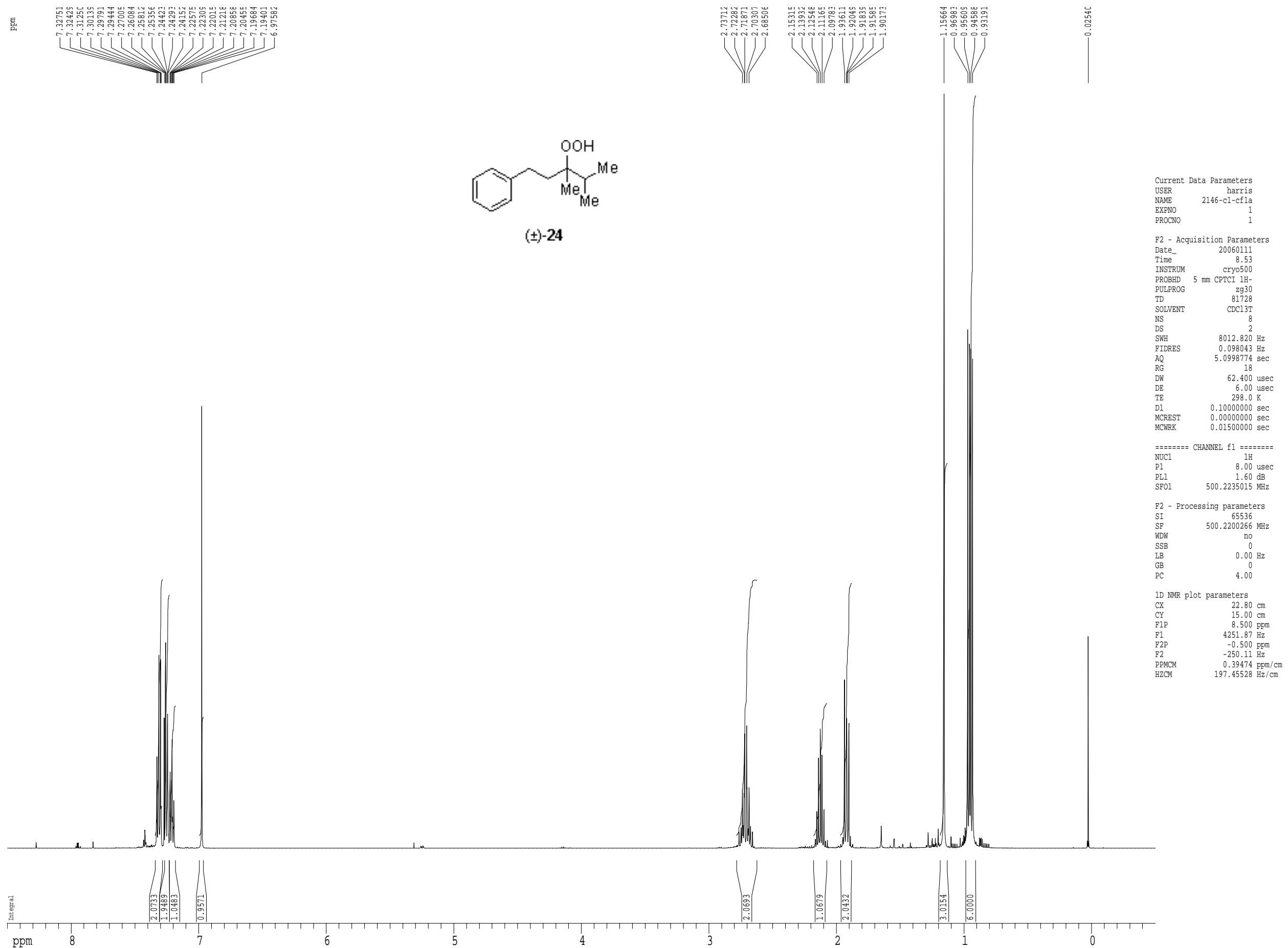
$13 \mathrm{C}$ spectrum with $1 \mathrm{H}$ decoupling
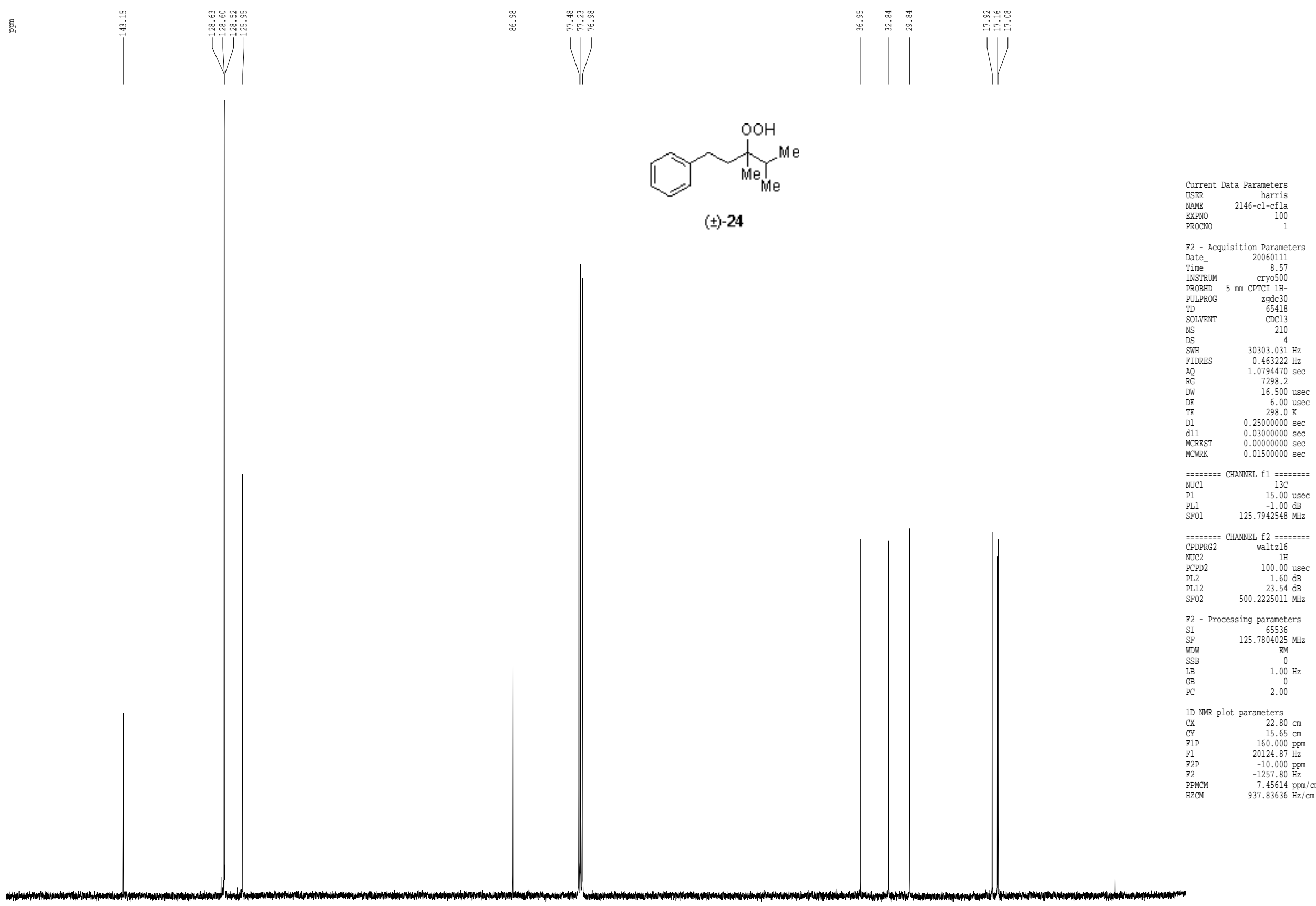
1H spectrum
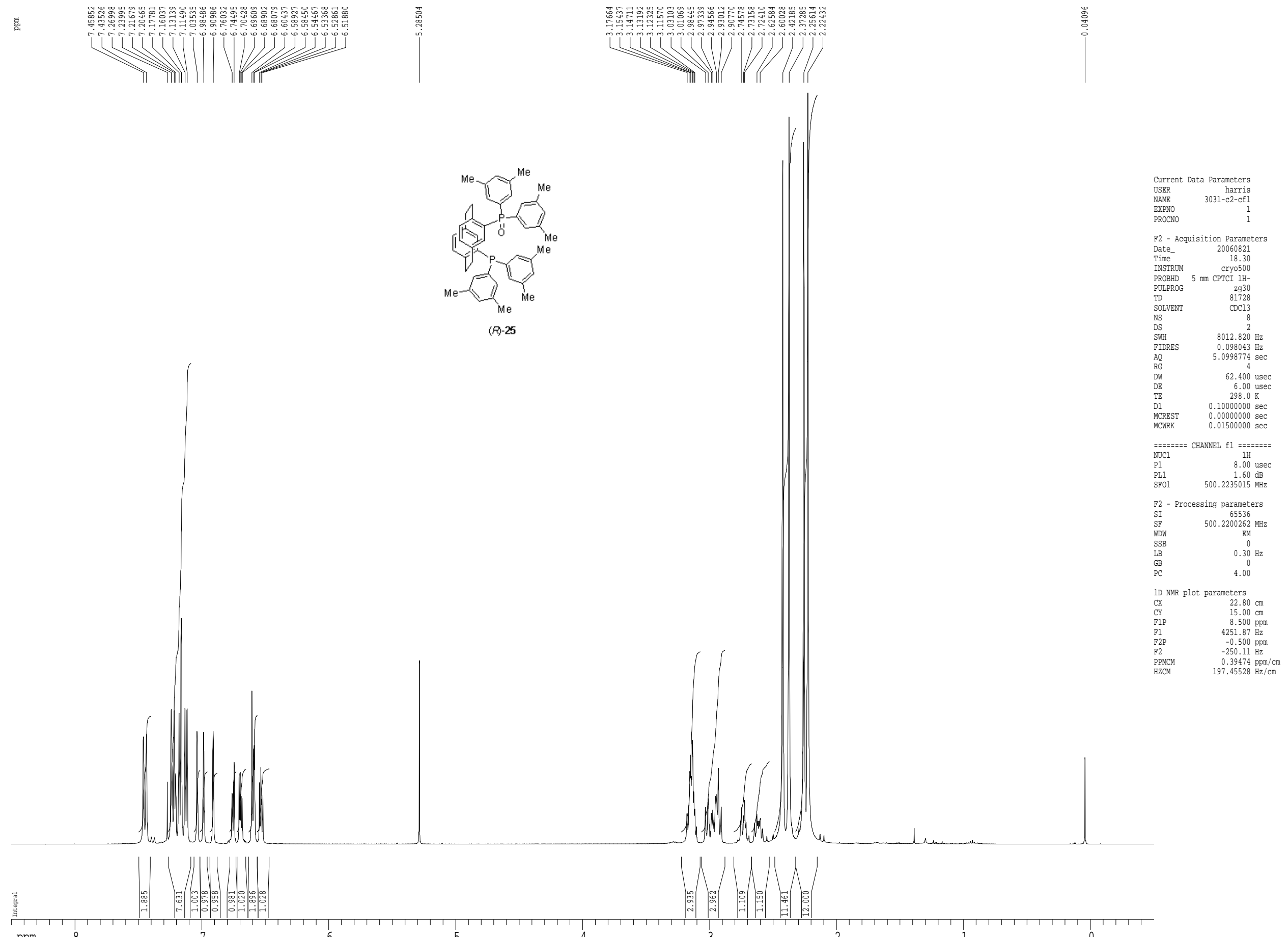
$13 \mathrm{C}$ spectrum with $1 \mathrm{H}$ decoupling

(1)
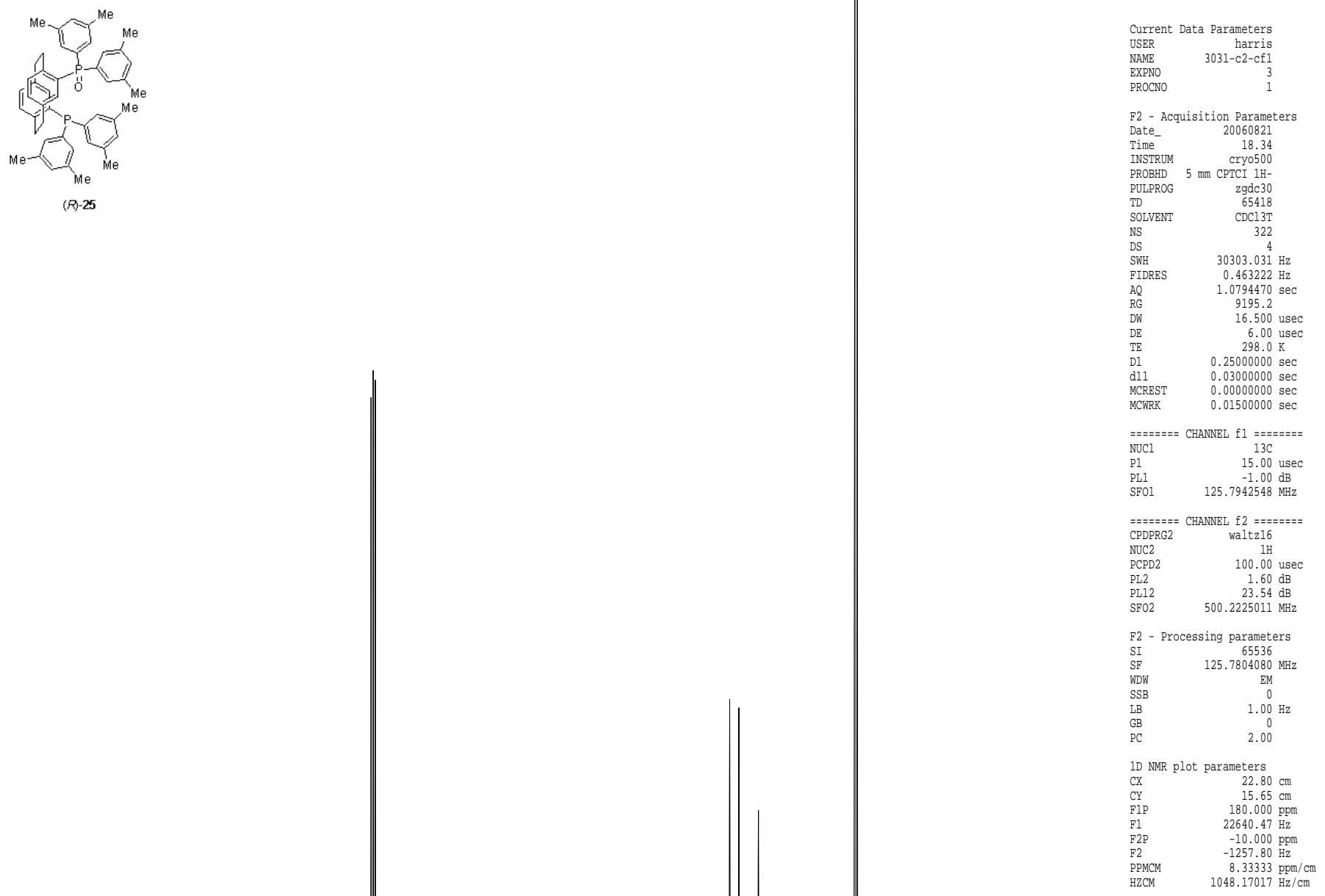
31P spectrum with $1 \mathrm{H}$ decoupling
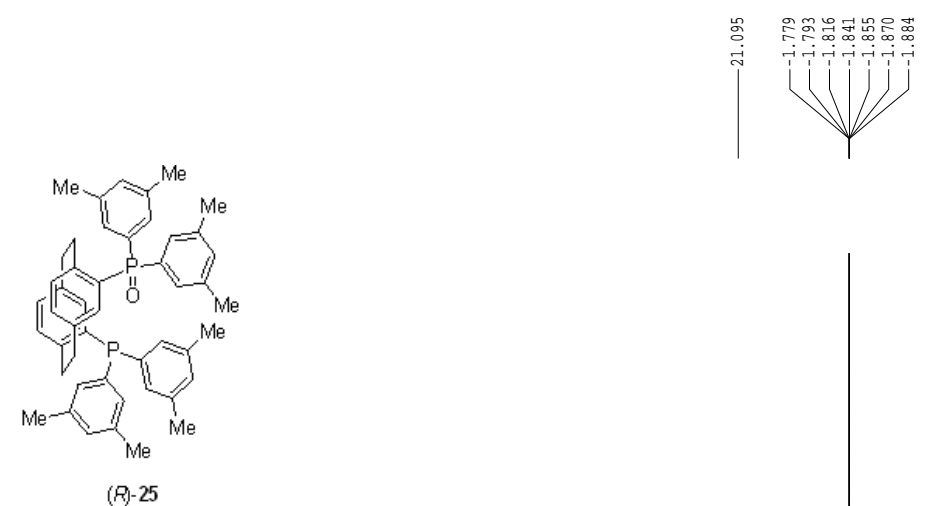

$(R)-25$

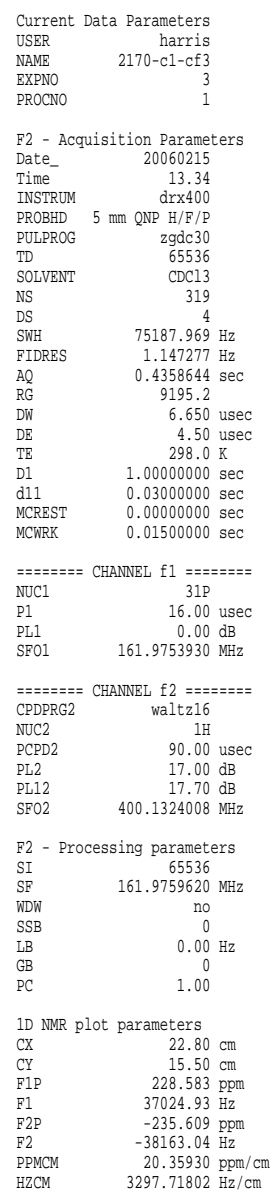


Supporting Information: Driver, Harris, Woerpel

1H spectrum
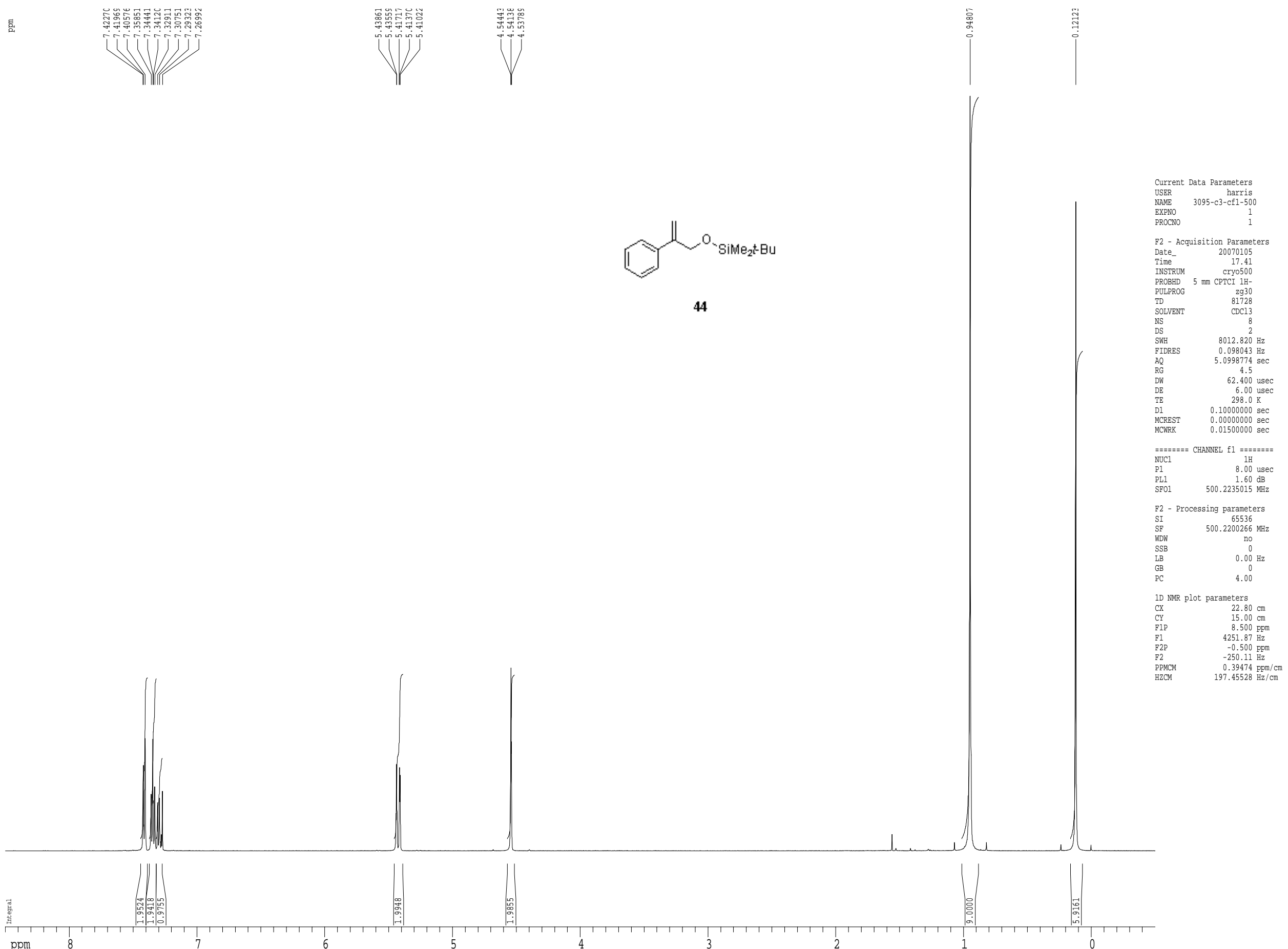

44 
Supporting Information: Driver, Harris, Woerpel

$13 \mathrm{C}$ spectrum with $1 \mathrm{H}$ decoupling

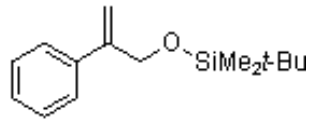


Supporting Information: Driver, Harris, Woerpel

1H spectrum

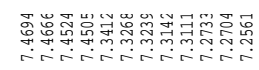

WWH

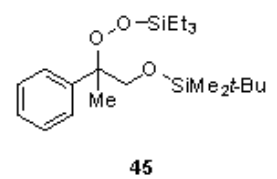

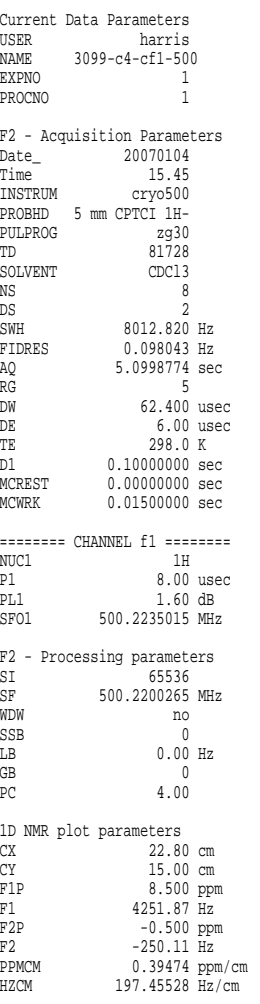

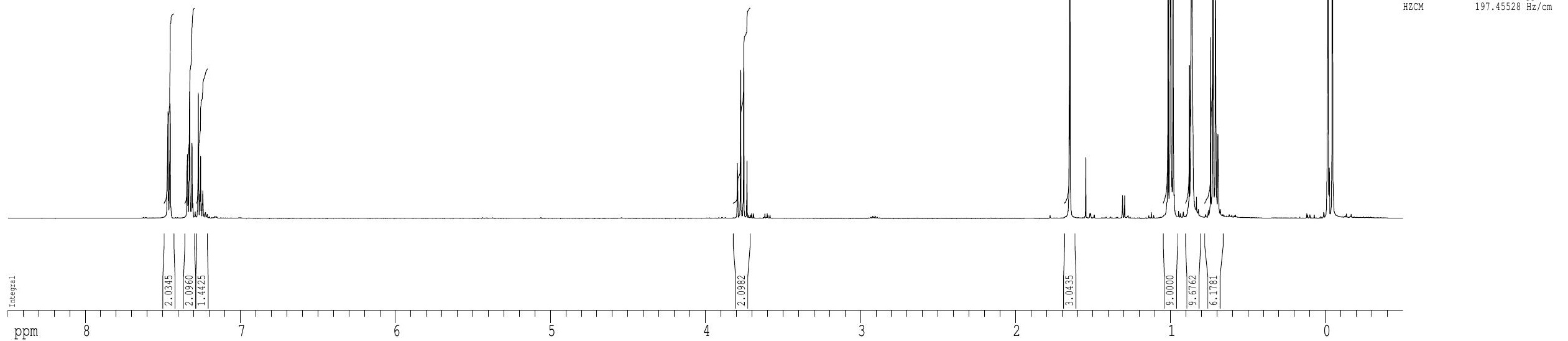


$13 \mathrm{C}$ spectrum with $1 \mathrm{H}$ decoupling

镸

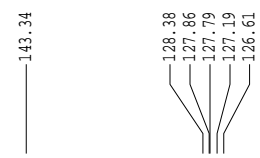

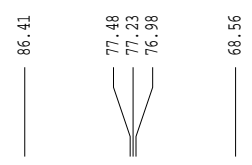

(1) ${ }_{m e}^{0} \operatorname{sime}_{2}$-Bu

45

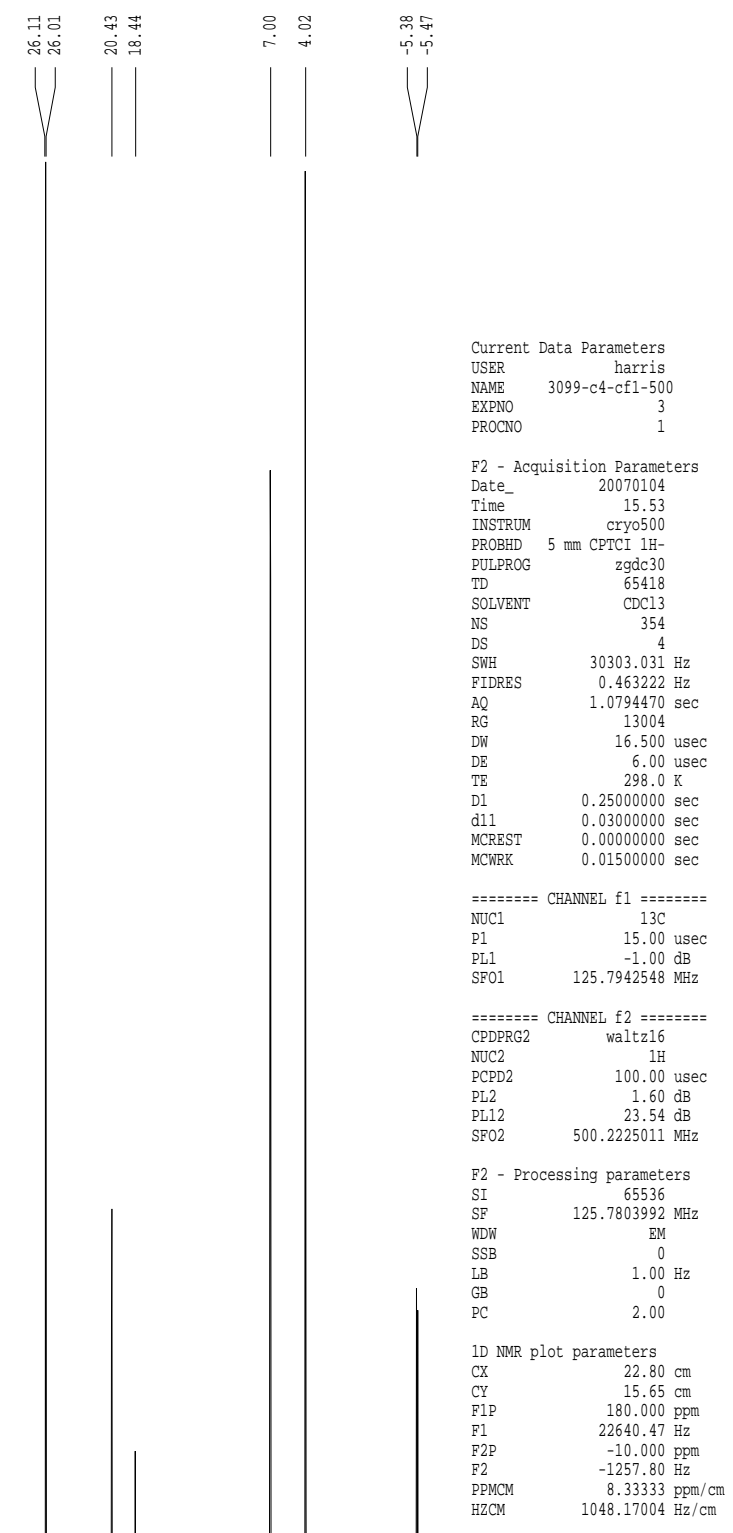

